\title{
ALMA data suggest the presence of spiral structure in the inner wind of CW Leonis ${ }^{\star}$
}

\author{
L. Decin ${ }^{1}$, A. M. S. Richards ${ }^{2}$, D. Neufeld ${ }^{3}$, W. Steffen ${ }^{4}$, G. Melnick ${ }^{5}$, and R. Lombaert ${ }^{1}$ \\ ${ }^{1}$ Instituut voor Sterrenkunde, Katholieke Universiteit Leuven, Celestijnenlaan 200D, 3001 Leuven, Belgium \\ e-mail: Leen.Decin@ster. kuleuven. be \\ 2 JBCA, Department Physics and Astronomy, University of Manchester, Manchester M13 9PL, UK \\ 3 The Johns Hopkins University, Baltimore, MD 21218, USA \\ 4 Instituto de Astronomía, Universidad Nacional Autónoma de Mexico, Apdo. Postal 877, 22800 Ensenada, BC, Mexico \\ 5 Harvard-Smithsonian Center for Astrophysics, 60 Garden Street, MS 66, Cambridge, MA 02138, USA
}

Received 13 July 2014 / Accepted 24 October 2014

\section{ABSTRACT}

\begin{abstract}
Context. Evolved low-mass stars lose a significant fraction of their mass through stellar winds. While the overall morphology of the stellar wind structure during the asymptotic giant branch (AGB) phase is thought to be roughly spherically symmetric, the morphology changes dramatically during the post-AGB and planetary nebula phase, during which bipolar and multi-polar structures are often observed.

Aims. We aim to study the inner wind structure of the closest well-known AGB star CW Leo. Different diagnostics probing different geometrical scales have implied a non-homogeneous mass-loss process for this star: dust clumps are observed at milli-arcsec scale, a bipolar structure is seen at arcsecond-scale, and multi-concentric shells are detected beyond $1^{\prime \prime}$.

Methods. We present the first ALMA Cycle 0 band 9 data around $650 \mathrm{GHz}(450 \mu \mathrm{m})$ tracing the inner wind of CW Leo. The fullresolution data have a spatial resolution of 0 ' $42 \times 0$ '.24, allowing us to study the morpho-kinematical structure of CW Leo within $\sim 6^{\prime \prime}$. Results. We have detected 25 molecular emission lines in four spectral windows. The emission of all but one line is spatially resolved. The dust and molecular lines are centered around the continuum peak position, which is assumed to be dominated by stellar emission. The dust emission has an asymmetric distribution with a central peak flux density of $\sim 2 \mathrm{Jy}$. The molecular emission lines trace different regions in the wind acceleration region and imply that the wind velocity increases rapidly from about $5 R_{\star}$, almost reaching the terminal velocity at $\sim 11 R_{\star}$. The images prove that vibrational lines are excited close to the stellar surface and that $\mathrm{SiO}$ is a parent molecule. The channel maps for the brighter lines show a complex structure; specifically, for the ${ }^{13} \mathrm{CO} J=6-5$ line, different arcs are detected within the first few arcseconds. The curved structure in the position-velocity (PV) map of the ${ }^{13} \mathrm{CO} J=6-5$ line can be explained by a spiral structure in the inner wind of CW Leo, probably induced by a binary companion. From modelling the ALMA data, we deduce that the potential orbital axis for the binary system lies at a position angle of $\sim 10-20^{\circ}$ to the north-east and that the spiral structure is seen almost edge-on. We infer an orbital period of $55 \mathrm{yr}$ and a binary separation of $25 \mathrm{au}$ (or $\sim 8.2 R_{\star}$ ). We tentatively estimate that the companion is an unevolved low-mass main-sequence star.

Conclusions. A scenario of a binary-induced spiral shell can explain the correlated structure seen in the ALMA PV images of CW Leo. Moreover, this scenario can also explain many other observational signatures seen at different spatial scales and in different wavelength regions, such as the bipolar structure and the almost concentric shells. ALMA data hence for the first time provide the crucial kinematical link between the dust clumps seen at milli-arcsecond scale and the almost concentric arcs seen at arcsecond scale.
\end{abstract}

Key words. stars: AGB and post-AGB - stars: mass-loss - circumstellar matter - binaries: general - stars: individual: CW Leo

\section{Introduction}

CW Leo (also known as IRC +10216) is the nearest carbon-rich asymptotic giant branch (AGB) star at a distance of about 120 to $150 \mathrm{pc}$ (Men'shchikov et al. 2001; Groenewegen et al. 2012, and reference therein). During the AGB phase, the evolution is determined by mass loss, with the mass-loss rate being significantly higher than the rate at which mass is comsumed by nuclear burning (Lagadec et al. 2008). The generally accepted idea is that mass loss proceeds in a two-step process: (1) pulsations lift photospheric layers to temperatures that are cool and dense enough for molecules to nucleate and form dust species; (2) radiation pressure on the dust grains pushes the material

\footnotetext{
$\star$ Appendix $\mathrm{A}$ is available in electronic form at http://www . aanda.org
}

outwards, and a wind is generated. However, even for the nearest carbon-rich AGB star it is still not well established whether the dominant behaviour of the mass loss occurs by homogeneous, isotropic processes, or if small-scale irregularities or instabilities dictate the overall appearance.

Specifically, for CW Leo it has been shown that at milliarcsecond scale dust clumps that formed in the dust formation region are moving as a result of the expanding wind of the star, with clump velocity motions varying between 7.9 and $17.5 \mathrm{~km} \mathrm{~s}^{-1}$ (Tuthill et al. 2000; Weigelt et al. 2002; Menut et al. 2007). Formation of new dust clumps at irregular time intervals might explain the fading illumination of some dust features during some periods. At arcsecond-scale, a bipolar morphology is seen in optical data with a bipolar axis at a position angle estimated at about $8^{\circ}$ (Mauron \& Huggins 2000) to $22^{\circ}$ (Leão et al. 2006; with respect to north). This feature probably 
indicates that scattering from stellar photons is more efficient in the polar direction. Evidence was provided from optical and near-infrared data for an equatorial density enhancement in the form of a geometrically thick disk (Dyck et al. 1987) or dusty torus (Skinner et al. 1998; Murakawa et al. 2005; Jeffers et al. 2014) seen almost edge-on. Beyond 1", multiple almost concentric shells (or arcs) are detected on top of the smooth extended envelope (Mauron \& Huggins 1999, 2000; Leão et al. 2006; Dinh-V-Trung \& Lim 2008; Fong et al. 2003; Decin et al. 2011). The nested shells are composed of thinner elongated arcs with a typical width of $\sim 1.6^{\prime \prime}$ and separated by $\sim 3^{\prime \prime}-20^{\prime \prime}$, corresponding to $\sim 150-1000 \mathrm{yr}$. These partial shells have a typical angular extent of $\sim 30^{\circ}$ to $90^{\circ}$ and are detected up to a distance of $320^{\prime \prime}$ (Decin et al. 2011). Around 450", Herschel data have revealed a bow shock (Ladjal et al. 2010).

In this paper, we present the first ALMA band 9 observations of the inner envelope of CW Leo at a spatial resolution of $\sim 0.2^{\prime \prime}\left(\sim 10 R_{\star}\right.$, with $1 R_{\star} \sim 5 \times 10^{13} \mathrm{~cm}$; De Beck et al. 2012). In Sect. 2 we describe the ALMA observations and data reduction including imaging and image fidelity. Section 3 reports on the astrometry and continuum properties. The ALMA spectra are described in Sect. 4, and the channel maps and PV-diagrams are shown in Sect. 5. A qualitative interpretation of the ALMA ${ }^{13} \mathrm{CO} J=6-5$ channel maps is given in Sect. 6. Preliminary morpho-kinematical modelling of the ALMA data is presented in Sect. 7. The results are discussed in Sect. 8, and the conclusions are given in Sect. 9.

\section{ALMA observations and data reduction}

\subsection{ALMA observations}

IRC +10216 was observed by ALMA on 1 December 2012 for proposal code 2011.0.00277.S (Cycle 0). The total time on IRC +10216 was $17.5 \mathrm{~min}$ spread over $35 \mathrm{~min}$. The precipitable water vapour was $0.55 \mathrm{~mm}$. The 18 antennas provided good data, with minimum and maximum baselines of 25-340 m.

Four $1.875-\mathrm{GHz}$ frequency bands (spectral windows, spw) were used for science, centred at 643.6645, 646.4827, 658.0587, and $661.1205 \mathrm{GHz}$ (ALMA Band 9). Each spectral window was divided into 3840 channels of $488 \mathrm{kHz}$. After Hanning smoothing, the effective velocity resolution is $0.455 \mathrm{~km} \mathrm{~s}^{-1}$. The usual ALMA instrumental corrections were applied, including those derived from water vapour radiometry and system temperature measurements. The Cycle 0 flux scale was derived with respect to Titan using a resolved, frequency-dependent model derived from its ephemeris ${ }^{1}$, as appropriate for the baseline lengths whose flux density was greater than the noise (maximum $\sim 6.535 \mathrm{Jy}$ ). The flux density of the QSO 3C 279 was derived with respect to Titan and found to be $(9.39,9.28,8.82$, 8.70) Jy in spw 0-3. QSO 3C 279 is point-like on all baselines and was used in turn to scale the antenna gains for all other sources. The flux density scale is formally accurate to $20 \%$ in band 9; the accuracy of our data might be even better because the flux densities derived for other calibration sources were consistent with values measured during other observations. The compact, bright QSO 3C 279 and J0854+201 were used as bandpass calibration and phase reference sources, respectively; both were also used to calibrate the pointing.

\footnotetext{
The so-called Butler-JPL-Horizons 2010 models, see ALMA Memo 594.
}

\subsection{Data reduction}

Normal data reduction procedures were performed in CASA $\left(\right.$ McMullin et al. 2007) ${ }^{2}$. Inspection of the phase reference source data showed that the phase of the first few scans could be connected smoothly, without wrapping, but there were apparent discontinuities in the second half of the data. We applied the phase-reference solutions to the target data and inspected the first half of the corrected data to identify the line-free channels. We used them to make a continuum image, which provided a model for self-calibration of all the target continuum data. The residual scatter in the final self-calibration solutions was about $5^{\circ}$ in phase and $10 \%$ in amplitude. Using the expressions from Perley (1999), this corresponds to a dynamic range limitation of $\sim 150-300$, depending on whether the errors are correlated between scans. We applied the solutions to all the channels of the target data. Finally, we made a preliminary cube to refine the selection of continuum channels, subtracted them, and made the final line and continuum images.

We used the software CASA for the Fourier transformation and to clean each channel to produce image cubes for all spectral windows. During this process, we corrected for the motion of Earth relative to the local standard of rest. For the identified lines, we extracted the channels covering that line and set the velocity to zero at its rest frequency. The noise in the image cubes is $0.2-0.3 \mathrm{Jy} \mathrm{beam}^{-1}$ per $488 \mathrm{kHz}$ channel $^{3}$. The noise is highest near the $658 \mathrm{GHz}$ atmospheric water line and is also higher in bright channels because of the dynamic range limitations and because it is slightly increased by continuum subtraction and residual calibration errors.

The short duration of the observations provided sparse visibility plane coverage, most noticeable at the highest spectral resolution. The full-resolution images were made with partial uniform weighting to give a synthesised beam 0. ' $42 \times 0.24$ for a position angle of $117^{\circ}$ (measured anticlockwise from north). This is similar to the natural beam position angle, but improves the resolution without increasing the noise appreciably ${ }^{4}$. A beam of 0 .' $^{\prime} \times 0$.' 4 was used for the three-channel averaged images, which have a resolution of $\sim 0.7 \mathrm{~km} \mathrm{~s}^{-1}$ and a noise value of $0.13-0.18 \mathrm{Jy} \mathrm{beam}^{-1}$ (see Sect. 5.1).

The shortest baselines in our observations impose a limit of $\sim 3^{\prime \prime}$ on the size of any structure that can be imaged accurately. Emission on scales $3^{\prime \prime}-6^{\prime \prime}$ is imperfectly sampled, and emission that is smoothly extended over $>6^{\prime \prime}$ is undetectable, even if it is much brighter than our limit ( $\sim 1 \mathrm{Jy} \mathrm{beam}^{-1}$ at full resolution) for compact emission.

These limitations (dynamic range and sparse visibility plane coverage) produce two kinds of artefacts. The dynamic range limitations are apparent as residual sidelobes of the synthesised beam: the intensity is proportional to the channel peak and is placed symmetrically w.r.t. the channel peak at a constant

\footnotetext{
2 http://casa.nra.edu - Credit: International consortium of scientists based at the National Radio Astronomical Observatory (NRAO), the European Southern Observatory (ESO), the National Astronomical Observatory of Japan (NAOJ), the CSIRO Australia Telescope National Facility (CSIRO/ATNF), and the Netherlands Institute for Radio Astronomy (ASTRON) under the guidance of NRAO.

3 The requested sensitivity of our Cycle 0 proposal was $0.04 \mathrm{Jy} \mathrm{beam}^{-1}$ per km, which would have required six times as long on-target.

4 Natural weighting simply takes each data point at the sampled position in the visibility plane; uniform weighting interpolates into the missing spacings, and the intermediate scheme used here gives more weight depending on the local sample density.
} 
spacing (scaled with frequency). We took care to check that any apparently symmetric structures were not in fact co-incident with sidelobes. The effects of poorly sampled emission are more complicated because they are the convolution of the actual distribution with the sidelobes. If the true distribution would for example be a disk $>6^{\prime \prime}$ across in a given channel, the inner $\sim 3^{\prime \prime}$ would be faithfully imaged, but at larger separations negative and positive ring-like artefacts (known as the "cereal-bowl" effect) might be visible. Nevertheless, smaller and larger rings (or patches of emission) seen in the channel maps with a size in the $3^{\prime \prime}-6$ " range result from actual structures with a smaller or larger size, respectively, which allows one to at least partly reconstruct the actual morphological structures.

\subsection{Position measurements}

We measured the positions and extents of emission by fitting 2D Gaussian components to the continuum peak and to the zeroth moment of each line (see Sect. 3). The noise-based uncertainty (for a sparsely filled snapshot) is given by the beam size divided by the signal-to-noise ratio ( $\mathrm{S} / \mathrm{N}$; before self-calibration), and gives the uncertainty in comparing positions within the same data set. However, if the actual flux distribution is non-Gaussian, the uncertainties are larger.

\section{Imaging results}

In this section, we discuss the astrometry and stellar and dust properties of CW Leo as deduced from the ALMA images in band 9 .

\subsection{Astrometry}

The position of the $650 \mathrm{GHz}$ continuum peak is 09:47:57.4553 $+13: 16: 43.749$ (J2000), epoch 2012.92. Four factors affect the astrometry. Uncertainties in the antenna positions and the phase reference source coordinates are smaller than a few mas. The noise-based uncertainty (for a sparsely filled snapshot) is given by the beam size divided by the signal-to-noise ratio (before selfcalibration), $\sim 0$ ' 02 for the continuum peak. The uncertainty in measuring the extent of emission is about twice the position uncertainty. The phase reference source is $14^{\circ}$ from IRC +10216 , and the timescales of phase changes due to the atmosphere lead to the dominant uncertainty of 0.25 . Menten et al. (2012) used the VLA in 2006.16 to measure the position and proper motion of IRC +10216, which would give 09:47:57.4417 +13:16:43.896 in 2012.92 (uncertainty $0{ }^{\prime} 012$ ). The discrepancy from the position measured by ALMA is $\left(0\right.$ ' $\left.20,-0{ }^{\prime} 15\right)$, which is within the uncertainties.

The position fitted to the zeroth moment of each line apart from ${ }^{13} \mathrm{CO}$ is within 30 mas (the combined position uncertainties) of the continuum peak; the mean position of all these lines is within 6 mas. The ${ }^{13} \mathrm{CO}$ peak is offset by $(-67,-37)$ mas, but this is probably due to the relatively poor fit of a Gaussian component to the complex and extended CO emission (see Sect. 5.1).

\subsection{Stellar and dust properties}

The total flux density within the $3 \sigma$ contour is $5.66 \mathrm{Jy}$, see Fig. 1. The continuum peak is $4.362 \mathrm{Jy} \mathrm{beam}^{-1}$ with a noise uncertainty of $0.023 \mathrm{Jy}$ beam $^{-1}$ and a calibration uncertainty $<0.87 \mathrm{Jy} \mathrm{beam}^{-1}$. The size (deconvolved from the beam) is $120 \pm 4$ mas by $108 \pm 5$ mas at a position angle (PA) of

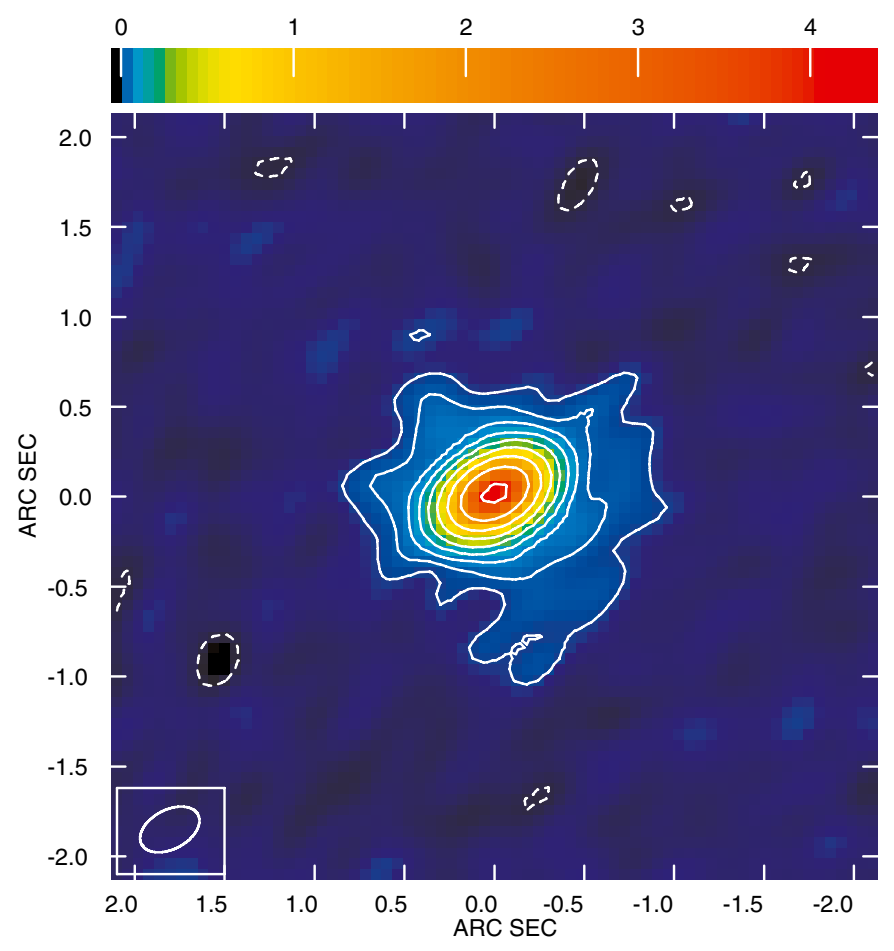

Fig. 1. Continuum image of CW Leo for a beam of 0 .' $^{\prime} 42 \times 00^{\prime} 24$ (shown as an ellipse in the bottom left corner). The contour levels are at $[-1,1$, $2,4,8,16,32,64,128,256] \times 15 \mathrm{mJy}^{\text {beam }}{ }^{-1}\left(3 \sigma_{\mathrm{rms}}\right)$.

$128 \pm 20^{\circ}$. The ALMA total flux density at $650 \mathrm{GHz}$ is slightly higher, although within the uncertainty limits, than the continuum emission flux density derived by Young et al. (2004) using the SMA at $680 \mathrm{GHz}$, who found a compact unresolved component in their $\sim 2$ " full width at half maximum (FWHM) beam with a flux density of $3.9 \pm 1.2 \mathrm{Jy}$.

The stellar photospheric emission contributes part of the total flux density at $650 \mathrm{GHz}$. Following Jenness et al. (2002), the ALMA data were taken near maximum light (at phase $\varphi=$ 0.98 ), which would imply a stellar luminosity of $\sim 15600 L_{\odot}$ according to De Beck et al. (2012). For an effective temperature of $2330 \mathrm{~K}$ (De Beck et al. 2012), this yields a stellar diameter of 48 mas. Groenewegen et al. (1997) reported a stellar diameter of 70.2 mas at $243 \mathrm{GHz}$ for an effective temperature of $T_{\text {eff }}$ of $2000 \mathrm{~K}$. Menten et al. (2012) found a diameter of 83 mas at $43 \mathrm{GHz}$. Thus, the star is probably unresolved at our spatial resolution.

We used two approaches to estimate the stellar flux contribution at $650 \mathrm{GHz}$. First, using the IRAM Plateau de Bure Interferometer (PdBI), Lucas \& Guélin (1999) found a "point source" at $89 \mathrm{GHz}(3.5 \mathrm{~mm})$ and $242 \mathrm{GHz}(1.3 \mathrm{~mm})$ with flux densities of $65 \pm 7 \mathrm{mJy}$ and $487 \pm 70 \mathrm{mJy}$, respectively. They identified the point source as being the photospheric emission. Using a spectral index of $1.96( \pm 0.04)$ as derived by Menten et al. (2006), this yields a stellar contribution of $3.3 \mathrm{Jy}$ at $650 \mathrm{GHz}$. The uncertainty on this value is estimated to be about $20 \%$ and arises from the variability of the pulsating star and the uncertainties on the spectral index. Second, the stellar contribution at $650 \mathrm{GHz}$ can also be calculated from the effective temperature and stellar diameter, under the assumption of a black-body spectrum. Using the values obtained by De Beck et al. (2012) at maximum luminosity (see previous paragraph), the stellar flux at $650 \mathrm{GHz}$ is $1.3 \mathrm{Jy}$, while the values derived by Groenewegen et al. (1997) yield a stellar flux of 2.3 Jy. The difference in stellar 
flux estimates mainly arises from the uncertainties in the angular diameter; the uncertainty on the effective temperature only gives an uncertainty in the flux of $0.2 \mathrm{Jy}$. These values for the stellar contribution are a factor $\sim 1.4-2.5$ lower than the value obtained by extrapolating the "point-source" values of Lucas \& Guélin (1999). This might mean that part of the "point-source" flux densities at $89 \mathrm{GHz}(3.5 \mathrm{~mm})$ and $243 \mathrm{GHz}(1.3 \mathrm{~mm})$ might not come from the central star, resulting in too high an estimate for the stellar contribution at $650 \mathrm{GHz}$ when using the data of Lucas \& Guélin (1999). Following Reid \& Menten (1997), Groenewegen (1997), Groenewegen et al. (1997), we suggest that free-free emission at wavelengths beyond $800 \mu \mathrm{m}$ might (at least partly) contribute to the high flux density values of Lucas \& Guélin (1999).

The estimated stellar flux density at $650 \mathrm{GHz}$ of $\sim 2.3 \mathrm{Jy}$ (adopting the parameters of Groenewegen et al. 1997) is significantly lower than the flux density of $5.66 \mathrm{Jy}$ obtained from the ALMA data. Since free-free emission is negligible at $650 \mathrm{GHz}$ (Groenewegen 1997), we conclude that the extended emission seen in the ALMA data is due to dust emission alone.

We estimated the $650 \mathrm{GHz}$ emission from dust by subtracting a 2.3 Jy point source, convolved with the natural synthesised beam, from the continuum image, leaving a residual $3.36 \mathrm{Jy}$ (see Fig. 2). The main sources of uncertainty are the $20 \%$ uncertainty on the ALMA data and the estimated stellar flux density. The residual emission is extended and clearly shows an asymmetric distribution, with a central peak flux density of $\sim 2 \mathrm{Jy}$. The lowest three-sigma contour in Fig. 2 corresponds to a position uncertainty $\sim 1 / 3$ of the beam size, so although the apparent southern bifurcation may be unreliable, the overall extension is real. The inner emission appears to be elongated in a direction similar to the $\sim 128^{\circ}$ position angle of the equatorial density enhancement detected in scattered light and polarimetry data (Skinner et al. 1998; Murakawa et al. 2005; Jeffers et al. 2014). However, this is close to the direction of elongation of the natural synthesised beam and may be coincidence. The faintest emission is, nonetheless, elongated in an almost orthogonal direction, at a position angle of $\sim 20^{\circ}$ and $200^{\circ}$, corresponding to the biconical cone detected by Skinner et al. (1998), Mauron \& Huggins (2000; see Sect. 6).

The dust nucleation zone starts around $5 R_{\star}$ (or 100-150 mas, Decin et al. 2010b). This is similar to the resolution of these images, but we cannot distinguish faint details this close to the strong stellar peak. The outer radius of the dust detectable by ALMA is set by the radius at which it becomes too diffuse or too cool to be detectable. Subtracting any realistic estimate for the stellar contribution (1.3-3.3 Jy) leaves an approximately flat or centrally peaked flux distribution within the inner $\sim 160$ mas radius, which means that the dust could be close to optically thick. If the stellar contribution were $\gtrsim 4 \mathrm{Jy}$, the dust emission would be characterized by a central hollow.

Using the continuum image after subtracting a $2.3 \mathrm{Jy}$ star, assuming that the dust is optically thin and for an opacity $\kappa_{\lambda}$ at $650 \mathrm{GHz}$ of $90 \mathrm{~cm}^{2} / \mathrm{g}$ (Mennella et al. 1998) and a dust temperature distributed as derived by Decin et al. (2010a), we obtain a dust mass within $0.8^{\prime \prime}$ of $3-6 \times 10^{-7} M_{\odot}$, corresponding to a dust mass-loss rate of $1.1-2.2 \times 10^{-8} M_{\odot} /$ yr. This estimate for the dust mass-loss rate is a factor $\sim 4$ lower than derived by Decin et al. (2010a) from modelling the spectral energy distribution. The large error margin is due to the flux scale uncertainty, the unknown stellar contribution, and the unknown composition of the dust and geometry in the inner regions. Assuming a constant dust velocity equal to the terminal velocity of $14.5 \mathrm{~km} \mathrm{~s}^{-1}$, this yields a dust optical depth of $\sim 0.1$. Since the dust velocity

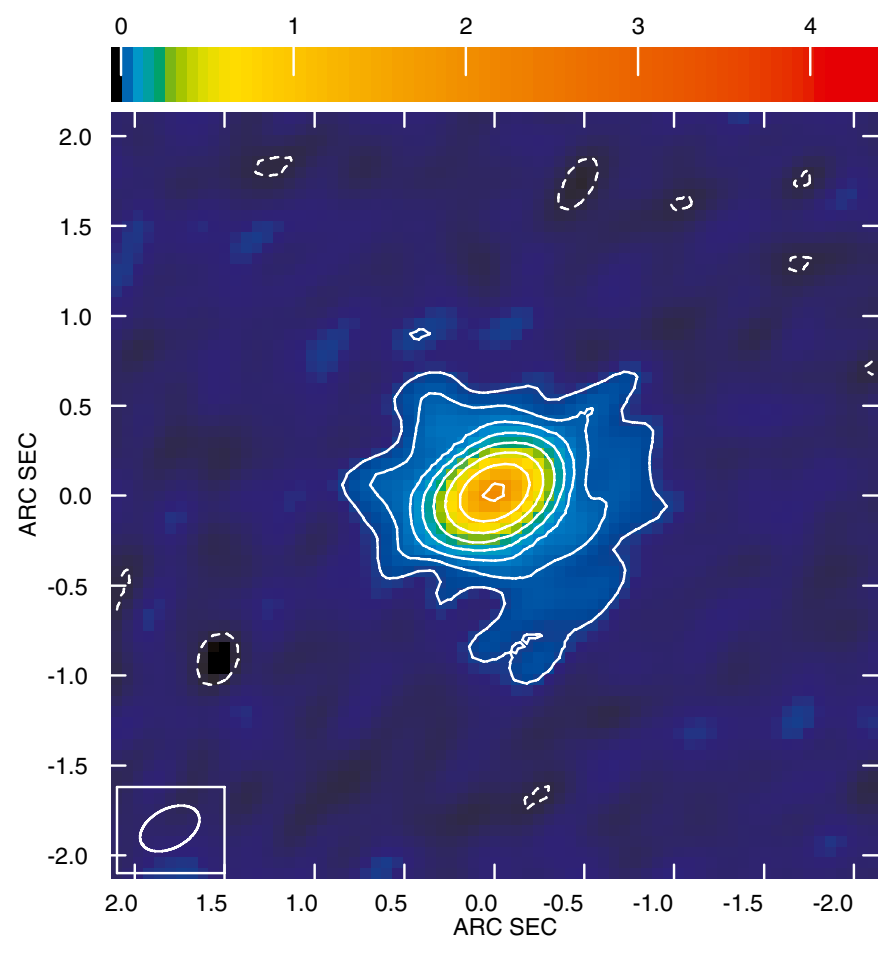

Fig. 2. Flux density after subtracting a $2.3 \mathrm{Jy}$ star. The contour levels are at $[-1,1,2,4,8,16,32,64,128] \times 15 \mathrm{mJy}_{\text {beam }}{ }^{-1}\left(3 \sigma_{\mathrm{rms}}\right)$.

might be overestimated in the wind acceleration region, the derived value for the dust optical depth might be an underestimate by a factor of a few. Future multi-wavelength high-resolution ALMA observations will provide a spectral index map and thus a better measurement of the optical depth.

\section{Spectral line results}

In this section, we present the ALMA spectra of CW Leo in band 9 around $650 \mathrm{GHz}$ (Sect. 4.1). For each detected emission line, we determine the line strength, line width, and spatial extension. The line strengths are compared with the line strengths as deduced from Herschel/HIFI observations in the same frequency window (Sect. 4.2). The line widths and spatial extension are used to determine the velocity structure of CW Leo in the inner wind region, where the wind is accelerated from the sound velocity to the terminal velocity (Sect. 4.3).

\subsection{ALMA spectra of CW Leo in band 9}

Figure 3 shows the spectra of the four spectral bands extracted using a circular aperture of $1.2^{\prime \prime}$ centred on the dust continuum peak. In the ALMA data, 25 molecular line transitions are detected, of which 7 are unidentified (see Table 1). The identified emission lines arise from ${ }^{13} \mathrm{CO}, \mathrm{C}^{18} \mathrm{O}, \mathrm{SiO},{ }^{29} \mathrm{SiO}, \mathrm{SiS},{ }^{29} \mathrm{SiS}$, ${ }^{30} \mathrm{SiS}, \mathrm{Si}^{33} \mathrm{~S}, \mathrm{Si}^{34} \mathrm{~S},{ }^{29} \mathrm{Si}^{34} \mathrm{~S}, \mathrm{SiC}_{2},{ }^{13} \mathrm{CS}$, and AlF.

Following De Beck et al. (2010), we applied a minimization routine to numerically fit the profiles of the molecular emission lines and extract some of the physical wind properties. We used the soft-parabola function (Olofsson et al. 1993), which provides the flux density $F$ as a function of velocity $v$ :

$F(v)=F_{\text {peak }}\left[1-\left(\frac{v-v_{\text {LSR }}}{v_{\text {line }}}\right)^{2}\right]^{\gamma / 2}$, 

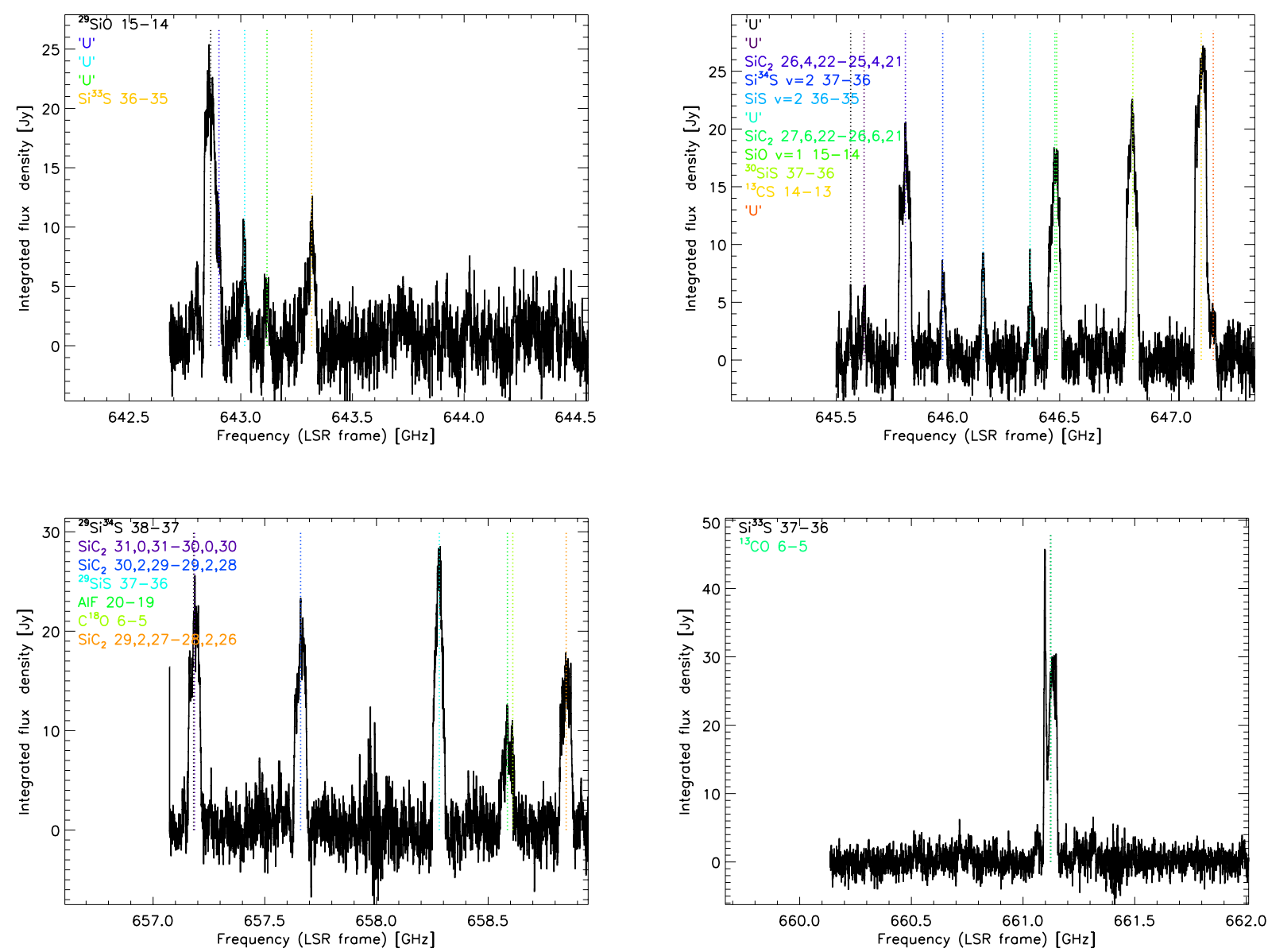

Fig. 3. Integrated flux density of the four spectral setups extracted in a circle of $1.2^{\prime \prime}$ in radius centred on the continuum peak. The molecular lines are indicated in colour; unidentified lines are marked as "U". Note that the noise around $658 \mathrm{GHz}$ is due to the strong atmospheric opacities at this frequency. The frequency values are corrected for the Earth's motion.

where $F_{\text {peak }}$ is the peak flux in the line centre, $v_{\text {LSR }}$ the systemic velocity with respect to the local standard of rest, $v_{\text {line }}$ the half width of the profile at continuum level, and $\gamma$ is a shape parameter. The results are listed in Table 1 . The softparabola fit to the ALMA data yields a mean difference between the rest frequencies and the ALMA frequencies of $-57.4 \mathrm{MHz}$ or $-26.41 \mathrm{~km} \mathrm{~s}^{-1}$, in accordance with the local standard of rest velocity derived by De Beck et al. (2012) from the high-spectral resolution Herschel/HIFI data. The value of the half line width, $v_{\text {line }}$, measures the gas velocity at the line formation region and will equal the terminal wind velocity $\left(v_{\infty}\right)$ for lines being formed in the region where the wind has achieved its full (terminal) velocity. The half-line width is determined by the expansion velocity, $v_{\exp }(r)$, and turbulent velocity, $v_{\text {turb }}(r)$, with $r$ being the radial distance. The turbulent velocity in stellar winds is not well established. Keady et al. (1988) proposed a turbulent velocity in the inner wind region of CW Leo of $\sim 5 \mathrm{~km} \mathrm{~s}^{-1}$ and a terminal turbulent velocity of $\sim 1.0 \mathrm{~km} \mathrm{~s}^{-1}$; De Beck et al. (2012) derived a value of $1.5 \mathrm{~km} \mathrm{~s}^{-1}$ from a fit to high-resolution Herschel/HIFI CO lines. The derived values for the half-line width range between $\sim 3.5 \mathrm{~km} \mathrm{~s}^{-1}$ and $\sim 15 \mathrm{~km} \mathrm{~s}^{-1}$. The velocity structure as derived from the ALMA data is discussed in detail in Sect. 4.3.

To determine the spatial extension, we computed the first moment for the inner $10 \mathrm{~km} \mathrm{~s}^{-1}$ of each line and measured the spatial FWHM by fitting a 2D Gaussian component and deconvolving the restoring beam (see Table 1). We only performed the spatial fitting to the inner $10 \mathrm{~km} \mathrm{~s}^{-1}$ to avoid line blending and since for an expanding shell the emission at line centre would be most spatially extended, whilst the extreme velocity emission comes from closer to the line of sight to the star. All lines have a high enough signal-to-noise ratio to be spatially resolved, except for the faintest unidentified line ("U" line) at $645.507 \mathrm{GHz}$. They are all compact enough for a good fit except for the ${ }^{13} \mathrm{CO}$ line, for which its apparent spatial FWHM, $s_{\text {FWHM }}$, represents only the inner peak. There is likely also to be extended $\mathrm{C}^{18} \mathrm{O}$ emission below our detection threshold. Table 1 also lists the (not deconvolved) largest angular size (LAS), that is, the maximum width of the area enclosed by the central contour at $2 \mathrm{Jy} \mathrm{km} \mathrm{s}{ }^{-1}$ perbeam (approximately $3 \sigma_{\mathrm{rms}}$ ). For ${ }^{13} \mathrm{CO} J=6-5$ there is also non-contiguous emission extended throughout the field of view (see Sect. 5.1). As illustrated in Fig. 4, the extension scales inversely with the upper state energy, $E_{\text {upper }}$. The anomalies in this plot are due to blending and the fact that the ALMA data resolve out a lot of the ${ }^{13} \mathrm{CO}$ flux (see Sect. 4.2).

\subsection{Comparison between ALMA and Herschel}

The frequency range observed with ALMA was also observed by the Herschel/HIFI instrument (de Graauw et al. 2010). A survey in all HIFI bands was carried out on May 11-15 2010. The data reduction and first results are described in Cernicharo et al. (2010). The HIFI beam size around $640 \mathrm{GHz}$ is 33.2" and the main-beam antenna efficiency is 0.75 (Roelfsema et al. 2012). The data were taken in double beam-switching mode 
Table 1. Spectral and spatial extent of the detected lines.

\begin{tabular}{|c|c|c|c|c|c|c|c|c|c|}
\hline & Molecule & Transition & $\begin{array}{c}v_{0} \\
{[\mathrm{GHz}]}\end{array}$ & $\begin{array}{c}E_{\text {upper }} \\
{[\mathrm{K}]} \\
\end{array}$ & $\begin{array}{c}S_{\text {int }} \\
\text { [Jy MHz] }\end{array}$ & $\begin{array}{l}s_{\mathrm{FWHM}} \\
{[\mathrm{mas}]}\end{array}$ & $\begin{array}{c}\text { LAS } \\
{[\operatorname{arcsec}]}\end{array}$ & $\begin{array}{c}F W H M \\
{\left[\mathrm{~km} \mathrm{~s}^{-1}\right]}\end{array}$ & $\begin{array}{c}v_{\text {line }} \\
{\left[\mathrm{km} \mathrm{s}^{-1}\right]}\end{array}$ \\
\hline 1 & ${ }^{29} \mathrm{SiO}$ & $15-14$ & $642.8080^{*}$ & 246 & $413(9)$ & $727(15)$ & 2.0 & $25.78^{*}$ & $14.36(0.26)^{*}$ \\
\hline 2 & "U” & & $642.845^{*}$ & - & $167(4)$ & 343 (14) & 1.2 & $25.78^{*}$ & $14.36(0.26)^{*}$ \\
\hline 3 & "U" & & 642.96 & - & $124(2)$ & $341(18)$ & 1.3 & 6.52 & $5.13(0.70)$ \\
\hline 4 & “U” & & 643.06 & - & $75(2)$ & $253(26)$ & 1.2 & 10.00 & $7.92(0.17)$ \\
\hline 5 & $\mathrm{Si}^{33} \mathrm{~S}$ & $36-35$ & 643.2608 & 570 & $143(2)$ & $386(14)$ & 1.3 & 17.81 & $15.19(0.80)$ \\
\hline 6 & “U” & & 645.507 & - & $39(2)$ & $<216$ & 0.7 & 5.33 & $3.57(0.92)$ \\
\hline 7 & "U" & & 645.567 & - & $64(2)$ & $235(36)$ & 0.9 & 7.60 & $4.95(0.80)$ \\
\hline 8 & $\mathrm{SiC}_{2}$ & $26,4,22-25,4,21$ & 645.7526 & 441 & $287(9)$ & $548(18)$ & 2.0 & 20.66 & $14.63(0.03)$ \\
\hline 9 & $\mathrm{Si}^{34} \mathrm{~S}$ & $v=237-36$ & 645.9198 & 2679 & $101(2)$ & $275(23)$ & 1.3 & 10.20 & $5.10(0.18)$ \\
\hline 10 & $\mathrm{SiS}$ & $v=236-35$ & 646.1000 & 2724 & $133(2)$ & 205 (19) & 1.1 & 7.89 & $3.94(0.12)$ \\
\hline 11 & "U" & & 646.31 & - & $79(2)$ & $282(29)$ & 1.5 & 5.49 & $3.61(0.98)$ \\
\hline 12 & $\mathrm{SiC}_{2}$ & $27,6,22-26,6,21$ & $646.4211^{*}$ & 501 & $295(6)$ & $512(16)$ & 1.7 & $19.15^{*}$ & $15.37(0.28)^{*}$ \\
\hline 13 & $\mathrm{SiO}$ & $v=115-14$ & $646.4296^{*}$ & 2018 & $300(9)$ & $558(18)$ & 2.0 & $19.15^{*}$ & $15.37(0.28)^{*}$ \\
\hline 14 & ${ }^{30} \mathrm{SiS}$ & $37-36$ & 646.7703 & 589 & $375(6)$ & $486(13)$ & 1.8 & 19.54 & $14.09(0.17)$ \\
\hline 15 & ${ }^{13} \mathrm{CS}$ & $14-13$ & 647.0762 & 233 & $492(13)$ & $675(16)$ & 1.9 & 22.10 & $15.29(0.08)$ \\
\hline 16 & "U" & & 647.13 & - & $71(2)$ & $246(39)$ & 1.1 & 10.80 & $7.46(0.95)$ \\
\hline 17 & ${ }^{29} \mathrm{Si}^{34} \mathrm{~S}$ & $38-37$ & $657.1243^{*}$ & 614 & 364 (13) & $593(23)$ & 2.1 & $20.56^{*}$ & $15.41(0.18)^{*}$ \\
\hline 18 & $\mathrm{SiC}_{2}$ & $31,0,31-30,0,30$ & $657.1290^{*}$ & 512 & 377 (13) & $568(23)$ & 2.2 & $20.56^{*}$ & $15.41(0.18)^{*}$ \\
\hline 19 & $\mathrm{SiC}_{2}$ & $30,2,29-29,2,28$ & 657.6034 & 509 & 340 (13) & $551(24)$ & 2.0 & 19.70 & $14.40(0.09)$ \\
\hline 20 & ${ }^{29} \mathrm{SiS}$ & $37-36$ & 658.2241 & 599 & $486(11)$ & $484(13)$ & 2.1 & 16.73 & $14.74(0.36)$ \\
\hline 21 & $\mathrm{AlF}$ & 20-19 & $658.5295^{*}$ & 331 & $158(6)$ & 424 (28) & 1.8 & $22.06^{*}$ & $17.08(0.46)^{*}$ \\
\hline 22 & $\mathrm{C}^{18} \mathrm{O}$ & $6-5$ & $658.5533^{*}$ & 111 & $100(9)$ & $526(54)$ & 1.5 & $22.06^{*}$ & $17.08(0.46)^{*}$ \\
\hline 23 & $\mathrm{SiC}_{2}$ & $29,2,27-28,2,26$ & 658.7924 & 503 & $280(13)$ & 579 (27) & 2.0 & 19.77 & $13.57(0.04)$ \\
\hline 24 & $\mathrm{Si}^{33} \mathrm{~S}$ & $37-36$ & $661.0665^{\dagger}$ & 602 & 407 (13) & $590(21)$ & 2.8 & $24.69^{\dagger}$ & $13.56(0.01)^{\dagger}$ \\
\hline 25 & ${ }^{13} \mathrm{CO}$ & $6-5$ & $661.0673^{\dagger}$ & 111 & $407(13)$ & $590(21)$ & 2.8 & $24.69^{\dagger}$ & $13.56(0.01)^{\dagger}$ \\
\hline
\end{tabular}

Notes. Column 1 designates a number to each transition; Cols. 2 and 3 list the molecular line; Col. 4 the line rest frequency $v_{0}$; Col. 5 the upper energy level $E_{\text {upper }}$; Col. 6 the integrated flux density of the fitted Gaussian component $S_{\text {int }}$ and its uncertainty between parentheses; Col. 7 the spatial FWHM $s_{\text {FWHM }}$ and its uncertainty; Col. 8 the LAS above $2 \mathrm{Jy} \mathrm{km} \mathrm{s}^{-1}$ per beam; Col. 9 the spectral FWHM, and the last column the half-line width $v_{\text {line }}{ }^{(*)}$ Blended within 2-20 $\mathrm{km} \mathrm{s}^{-1}$; ${ }^{(\dagger)}$ blended within $0.4 \mathrm{~km} \mathrm{~s}^{-1}$.

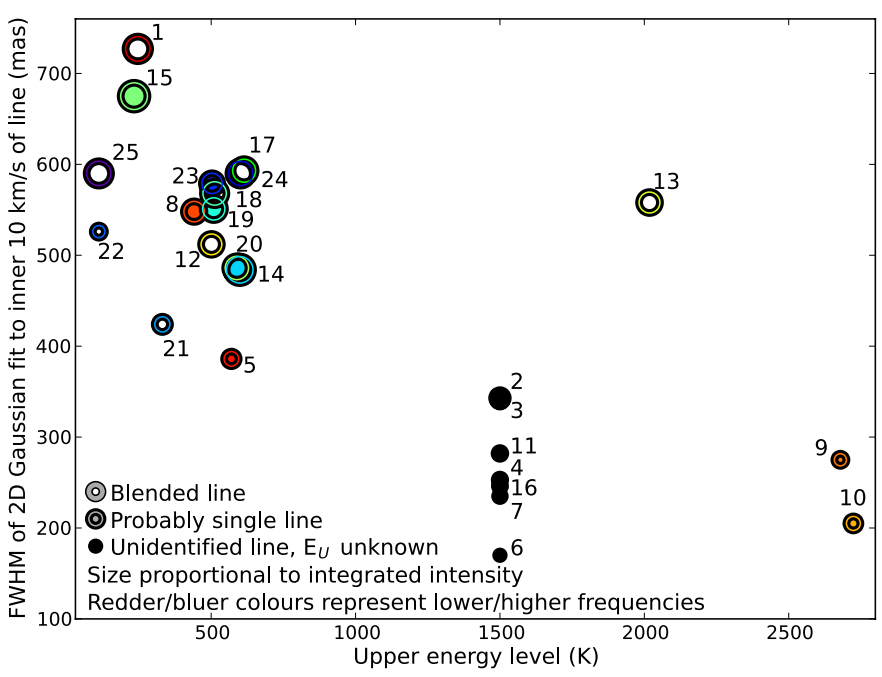

Fig. 4. Spatial FWHM as a function of the upper state energy level. Lines are identified by their number given in Col. 1 of Table 1 . The size of the symbols is proportional to the integrated intensity; unidentified lines are placed at an artifical upper state energy of $1500 \mathrm{~K}$.

at a frequency resolution of $1.1 \mathrm{MHz}$. To increase the $\mathrm{S} / \mathrm{N}$ of the weaker lines, some spectral regions were rebinned. The rms noise after averaging all scans is given in Table 2. To convert from antenna temperatures (in $\mathrm{K}$ ) to fluxes in Jy, we used the point-source sensitivity of $466 \mathrm{Jy} / \mathrm{K}$ (Roelfsema et al. 2012). The source size of all transitions listed in Table 2 can be approximated with a point source within the Herschel beam, with the exception of the ${ }^{13} \mathrm{CO} J=6-5$ line. Based on observations with the JCMT and CSO (Crosas \& Menten 1997) and our modelling of the ${ }^{13} \mathrm{CO}$ emission presented in Decin et al. (2010b) and De Beck et al. (2012), we derive a source size of $\sim 10^{\prime \prime}$ for the ${ }^{13} \mathrm{CO} J=6-5$ line, yielding a $3 \%$ correction w.r.t. point-source sensitivity.

Table 2 lists the integrated line intensities measured with Herschel and ALMA. Note that the ALMA integrated line intensities are higher than the values listed in Table 1 because the spatial fitting used for the values in Table 1 was restricted to the inner $10 \mathrm{~km} \mathrm{~s}^{-1}$ (see previous section). As can be seen from Table 2, only a few lines are not plagued by blends with other molecular line transitions. The half-line width at continuum level, $v_{\text {line }}$, of all common unblended lines agrees for both instruments. The integrated flux density of the unblended lines measured from the Herschel data is typically 1.2-1.7 times higher than that measured using ALMA (note that the overall ALMA flux scale is uncertain by up to $20 \%$ ). The ratio is lower for the $\mathrm{Si}^{33} \mathrm{~S} J=36-35$ transition, but this line has a low signalto-noise ratio in the Herschel data, and the ratio is much higher for ${ }^{13} \mathrm{CO} J=6-5$ line. The $20-50 \%$ additional flux detected by Herschel compared with ALMA can be explained by two factors.

Firstly, there are observational differences. Herschel is fundamentally more sensitive to large-scale emission. Its $5 \sigma$ sensitivity for these observations, per $0.7 \mathrm{~km} \mathrm{~s}^{-1}$, was $\sim 20 \mathrm{Jy} \mathrm{beam}^{-1}$, compared with $<0.2 \mathrm{Jybeam}^{-1}$ for ALMA. However, the Herschel beam is $\sim 10^{4}$ times the area of the ALMA synthesised beam. A $20 \mathrm{Jy}$ point source would be very bright in the ALMA data, but would be spread out over an area of more than $333^{\prime \prime 2} 2$ 
Table 2. Comparison between the Herschel and ALMA integrated line fluxes.

\begin{tabular}{|c|c|c|c|c|c|c|c|c|}
\hline Molecule & Transition & $\begin{array}{l}\text { Herschel } \\
T_{\mathrm{a}}^{\text {peak }} \\
(\mathrm{K})\end{array}$ & $\begin{array}{l}\text { Herschel } \\
\text { rms } \\
(\mathrm{mK})\end{array}$ & $\begin{array}{l}\text { Herschel } \\
S / N\end{array}$ & $\begin{array}{l}\text { Herschel } \\
F_{v} \mathrm{~d} v \\
(\mathrm{Jy} \mathrm{MHz})\end{array}$ & $\begin{array}{l}\text { ALMA } \\
F_{v} \mathrm{~d} v \\
(\mathrm{Jy} \mathrm{MHz})\end{array}$ & $\begin{array}{l}\text { Comments } \\
\text { Herschel } \\
\text { data }\end{array}$ & $\begin{array}{l}\text { Comments } \\
\text { ALMA } \\
\text { data }\end{array}$ \\
\hline${ }^{29} \mathrm{SiO}$ & $15-14$ & 0.065 & 9 & 7 & (1408) & $(834)$ & & blend " $U$ " \\
\hline $\mathrm{Si}^{33} \mathrm{~S}$ & $36-35$ & 0.047 & 8.9 & 5 & 210 & 271 & low $\mathrm{S} / \mathrm{N}$ & \\
\hline $\mathrm{SiC}_{2}$ & $26,4,22-25,4,21$ & 0.054 & 5.5 & 10 & 1168 & 816 & & \\
\hline $\operatorname{SiS} v=2$ & $36-35$ & 0.018 & 3.3 & 5 & 191 & 159 & low $\mathrm{S} / \mathrm{N}$ & \\
\hline $\mathrm{SiC}_{2}$ & $27,6,22-26,6,21$ & - & 4.5 & - & - & - & blend $\mathrm{SiO} v=1$ & blend $\mathrm{SiO} v=1$ \\
\hline $\mathrm{SiO} v=1$ & $15-14$ & - & 2.9 & - & - & - & blend $\mathrm{SiC}_{2}$ & blend $\mathrm{SiC}_{2}$ \\
\hline${ }^{30} \mathrm{SiS}$ & $37-36$ & 0.055 & 4.9 & 11 & 1035 & 950 & & \\
\hline${ }^{13} \mathrm{CS}$ & $14-13$ & 0.093 & 4.7 & 20 & 2012 & 1397 & & weak blend "U" \\
\hline${ }^{29} \mathrm{Si}^{34} S$ & $38-37$ & - & 7 & - & - & - & blend $\mathrm{SiC}_{2}$ & blend $\mathrm{SiC}_{2}$ \\
\hline $\mathrm{SiC}_{2}$ & $31,0,31-30,0,30$ & - & 5.1 & - & - & - & blend ${ }^{29} \mathrm{Si}^{34} \mathrm{~S}$ & blend ${ }^{29} \mathrm{Si}^{34} \mathrm{~S}$ \\
\hline $\mathrm{SiC}_{2}$ & $30,2,29-29,2,28$ & 0.061 & 8.4 & 7 & 1297 & 883 & & \\
\hline${ }^{29} \mathrm{SiS}$ & $37-36$ & 0.075 & 6.2 & 12 & 1452 & 1059 & & \\
\hline AlF & $20-19$ & - & 6.4 & - & - & - & blend $\mathrm{C}^{18} \mathrm{O}$ & blend $\mathrm{C}^{18} \mathrm{O}$ \\
\hline $\mathrm{C}^{18} \mathrm{O}$ & $6-5$ & - & 9 & - & - & - & blend AlF & blend AlF \\
\hline $\mathrm{SiC}_{2}$ & $29,2,27-28,2,26$ & 0.055 & 5.6 & 10 & 1281 & 735 & & \\
\hline${ }^{13} \mathrm{CO}$ & $6-5$ & 1.235 & 7 & 176 & 32525 & 1366 & weak blend $\mathrm{Si}^{33} \mathrm{~S} J=37-36$ & blend $\mathrm{Si}^{33} \mathrm{~S} J=37-36$ \\
\hline
\end{tabular}

Notes. Columns 1 and 2 list the molecule and its transition; Cols. 3-5 give the Herschel antenna peak temperature, rms and S/N; the Herschel and ALMA integrated line fluxes are tabulated in Col. 6; comments on blends as seen in the Herschel and ALMA data sets are given in the last two columns. The integrated line fluxes plagued by weak line blends are given within parentheses. A "-" indicates the cases of strong line blending where only detailed modelling can reveal the contribution of the individual components. The Herschel point-source sensitivity of $466 \mathrm{Jy} / \mathrm{K}$ (Roelfsema et al. 2012) has been used for all lines. For ${ }^{13} \mathrm{CO} J=6-5$, the estimated source size of $10^{\prime \prime}$ (Crosas \& Menten 1997) was taken into account.

in diameter, the surface brightness per ALMA beam would be below the detection limit. In addition, even brighter emission that is smooth on scales $>3^{\prime \prime}$ would be poorly imaged by ALMA, and not detected at all, however bright, if it were $>6^{\prime \prime}$, as explained in Sect. 2.2. Our results are consistent with this because the fraction of the Herschel flux detected by ALMA is higher for higher excitation temperature lines and lower for more spatially extended species, and we detect a similar fraction of the flux with ALMA for similar transitions. Specifically, for the transitions listed in Table 2, the estimated emission region for the SiS isotopologues ( $J=37-36$ or $J=36-35)$ in the groundvibrational state is $<6{ }^{\prime \prime} 5$ (based on modelling by Decin et al. $2010 b)$. For the ${ }^{13} \mathrm{CS} J=14-13$ line the main emission region is within $\sim 7$ " (based on the ${ }^{12} \mathrm{CS} J=7-6$ and ${ }^{13} \mathrm{CS} J=7-6$ line observed by Williams \& White 1992; Patel et al. 2011), and the ${ }^{12}$ CS $J=14-13$ SMA data of Young et al. (2004) even indicate an emission region within $\sim 4^{\prime \prime}$. As mentioned above, the main emission region for the ${ }^{13} \mathrm{CO} J=6-5$ transition is $\sim 10^{\prime \prime}$ and the ALMA observations have resolved out at least $95 \%$ of the flux. No flux will be resolved out for the high-excitation $\operatorname{SiS} v=2$ $J=36-35$ line with an upper state energy of $2724 \mathrm{~K}$; the difference between ALMA and Herschel integrated line flux values is within the calibration uncertainties of both instruments.

Secondly, the variability of thermal line emission due to the variability of the central star is different. Following Jenness et al. (2002), the Herschel and ALMA data were taken at phase 0.46 and 0.98 , respectively. The changes in the radiation field during the pulsation period can influence the molecular excitation. The infrared radiation field is expected to mainly affect molecules with strong vibrational transitions, with stronger modulations for lines excited close to the star. Using Herschel data, Teyssier et al. (2013) have recently shown that the ${ }^{12} \mathrm{CS} J=14-13$ has a maximum amplification factor in the line intensities of $\sim 1.3$, while for $\operatorname{SiS} J=37-36$ and $J=36-35$ this is $\sim 1.2$. The low-excitation $\mathrm{CO}$ lines seem quite insensitive to a change in the infrared radiation field.
Predicting whether a line will have a higher or lower amplification factor depending on the variability phase at the time of the observations is complex, however. The ALMA data are taken near light maximum and the Herschel data near light minimum, which indicates that higher excitation levels might be more populated at the time of the ALMA observations. However, the exact effect is dependent on the transition-rate probabilities with the connecting levels.

\subsection{Gas velocity structure in the inner wind region}

Table 1 and Fig. 5 show that the wind velocity of the lines falls into two groups. All but two of the identified lines trace a velocity higher than $13.5 \mathrm{~km} \mathrm{~s}^{-1}$ at a LAS radius (representing the not-deconvolved largest detectable scale) beyond 600 mas radius (or $30 R_{\star}$ ) and a spatial FWHM (illustrating the main line formation region) beyond 200 mas $\left(10 R_{\star}\right)$, indicating that the wind has reached its terminal velocity $\left(\sim 14.5 \mathrm{~km} \mathrm{~s}^{-1}\right)$ within $\sim 10 R_{\star}$ from the central star, in agreement with the results of Keady et al. (1988). The other group has line width velocities $<8 \mathrm{~km} \mathrm{~s}^{-1}$ within 800 mas LAS radius $\left(40 R_{\star}\right.$ ). This contains the two identified lines in $v=2$ excited vibrational states and all unblended "U" lines, suggesting that these also come from high-excitation states. The narrowest line is the "U" line at $646.31 \mathrm{GHz}$ with a velocity of only $3.6 \mathrm{~km} \mathrm{~s}^{-1}$, slightly higher than the sound velocity of $\sim 3 \mathrm{~km} \mathrm{~s}^{-1}$ at the base of the dust nucleation region.

Plotting the velocities as a function of the spatial FWHM (see Fig. 5) shows that the measured velocities rapidly increase from the sound velocity to $\sim 8 \mathrm{~km} \mathrm{~s}^{-1}$ between $\sim 100$ to 200 mas $\left(5-10 R_{\star}\right)$. Assuming that the turbulent velocity does not vary substantially in this small region, this indicates that carbonaceous material condenses around $5 R_{\star}$, resulting in a rapid acceleration of the gas until a velocity of $\sim 8 \mathrm{~km} \mathrm{~s}^{-1}$. In the four ALMA spectral windows, no lines are detected with a velocity between 8 and $13.5 \mathrm{~km} \mathrm{~s}^{-1}$, but the data suggest a rapid 

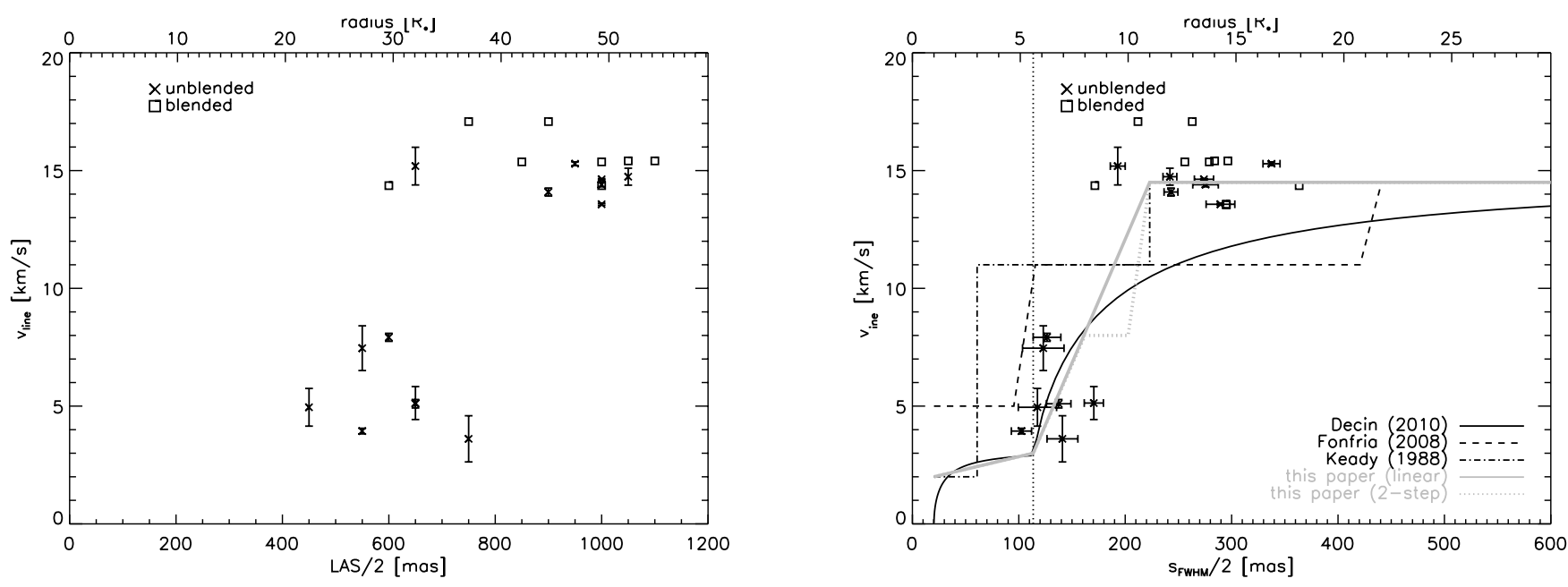

Fig. 5. Measured wind velocities (as determined from the half-line width $v_{\text {line }}$ ) versus half of the LAS (representing the not-deconvolved largest detectable scale of each transition) in the left panel and half of the spatial FWHM $s_{\mathrm{FWHM}}$ (representing the dominant line formation region) in the right panel. Note that the value for the LAS is restricted by the sensitivity limit of the observations and the fact that large-scale flux might be resolved out (see Sect. 2.2). The blended lines are indicated with a squared box, the unblended lines with a cross (and include the error bars). In the right panel, the full black line shows the velocity structure as derived by Decin et al. (2010a) from solving the momentum equation; the dashed line indicates the velocity structure as derived by Fonfría et al. (2008) from modelling different $J$ ro-vibrational transitions of $\mathrm{C}_{2} \mathrm{H}_{2}$, the dashed-dotted line the velocity structure derived by Keady et al. (1988) from the analysis of the near-infrared ro-vibrational CO spectrum, and the thick grey lines the acceleration derived from the ALMA data. The vertical dotted line indicates the dust nucleation region around 5.6 $R_{\star}$, where the sound velocity is $\sim 3 \mathrm{~km} \mathrm{~s}^{-1}$.

increase of the gas velocity from $\sim 8 \mathrm{~km} \mathrm{~s}^{-1}$ to $\sim 14.5 \mathrm{~km} \mathrm{~s}^{-1}$ around $\sim 10-12 R_{\star}$. This acceleration profile is not captured by the solution of the wind momentum equation derived by Decin et al. (2010a), who assumed instantaneous condensation of amorphous carbon, iron, silicon carbide, and magnesium sulfide dust species at a radius of $5.6 R_{\star}$. Keady et al. (1988) suggested a two-step acceleration process from an analysis of high-resolution near-infrared ro-vibrational CO spectra. They suggested an increase around 3 and $11 R_{\star}$, with the first increase up to a velocity of $11 \mathrm{~km} \mathrm{~s}^{-1}$ being due to the condensation of carbon and a second increase that might be linked to dust condensation involving $\mathrm{Mg}$ and/or $\mathrm{S}$. This idea was used and adapted by Fonfría et al. (2008), who modelled a large set of $\mathrm{C}_{2} \mathrm{H}_{2}$ lines. They suggested a strong velocity gradient from 5 to $11 \mathrm{~km} \mathrm{~s}^{-1}$ around $4.7-5.7 R_{\star}$ and a second velocity increase to $14.5 \mathrm{~km} \mathrm{~s}^{-1}$ around $20.75-21.75 R_{\star}$ (see Fig. 5). Based on the ALMA band 9 data, the wind acceleration profile can be further refined. The data can be fitted assuming a linear velocity increase from around $2-3 \mathrm{~km} \mathrm{~s}^{-1}$ at $\sim 5 R_{\star}$ reaching terminal velocity around $11 R_{\star}$ (see the full grey line in the right panel of Fig. 5). Assuming a two-step acceleration process, we obtain

$v(r)= \begin{cases}2-3 \mathrm{~km} \mathrm{~s}^{-1}, & 1 \leq r / R_{*}<5.6 \\ 3-8 \mathrm{~km} \mathrm{~s}^{-1}, & 5.6 \leq r / R_{*}<8 \\ 8 \mathrm{~km} \mathrm{~s}^{-1}, & 8 \leq r / R_{*}<10 \\ 8-14.5 \mathrm{~km} \mathrm{~s}^{-1}, & 10 \leq r / R_{*}<11 \\ 14.5 \mathrm{~km} \mathrm{~s}^{-1}, & 11 \leq r / R_{*},\end{cases}$

that is, a first jump around $5.6 R_{\star}$ and a second one around $10 R_{\star}$ (see the dotted grey line in the right panel of Fig. 5). To improve upon the solution of the momentum equation as derived by Decin et al. (2010a), different possibilities exist. (1) Either the dust absorption efficiencies need to increase in the nearinfrared, where the bulk of the stellar photons are emitted. (2) Or larger grains $(\gtrsim 0.2 \mu \mathrm{m})$ should be formed more efficiently close to the star, thus enhancing the wind acceleration via scattering processes (Höfner 2008). (3) A third possibility might exist by adapting the velocity at the start of the dust condensation region, which is now assumed to be the sound velocity (Decin et al. 2006). However, in that case, the dust-to-gas ratio would need to be decreased to avoid overshooting the observed terminal velocity.

\section{Channel maps and PV diagrams}

In Sects. 5.1 and 5.2, we show and discuss the channel maps and position-velocity (PV) diagrams of one representative high-excitation line with an upper state energy $\left(E_{\text {upper }}\right)$ around $2700 \mathrm{~K}$, of one medium-excitation line $\left(E_{\text {upper }} \sim 450 \mathrm{~K}\right)$, and of one low-excitation line $\left(E_{\text {upper }} \sim 100 \mathrm{~K}\right)$. The vibrationally excited line traces the wind acceleration region, molecules in the inner envelope with excitation energies of about a few hundred $\mathrm{K}$ are slightly resolved with the ALMA beam of $\sim 0.2^{\prime \prime}$, while the ${ }^{13} \mathrm{CO} J=6-5$ line shows a complex emission pattern.

\subsection{Channel maps and morphology}

As expected, the channel map of a very high-excitation line of a molecule in the inner wind region just above the photosphere, such as $\operatorname{SiS} v=2 J=36-35$ (Fig. 6), shows that the emission of this line comes from the very inner regions of the envelope, close to the stellar surface, where the wind material has a velocity of about $4 \mathrm{~km} \mathrm{~s}^{-1}$ and the gas kinetic temperature is typically around $900-1300 \mathrm{~K}$. The morphology in the region $<1^{\prime \prime}$ (or diameter of $50 R_{\star}$ ) is still more or less circular (for a circular beam of 0.4$)$.

The channel maps of molecular transitions formed in the inner wind region and with upper state energies of about a few hundred $\mathrm{K}$, such as the $\mathrm{SiC}_{2} 26(4,22)-25(4,21)$ line displayed in Fig. 7, show that the emission of this line is extended and displays a non-homogeneous distribution of the molecular density. The typical spatial FWHM of these lines is around a few hundred 
L. Decin et al.: Edge-on spiral detected with ALMA in the inner wind of CW Leo
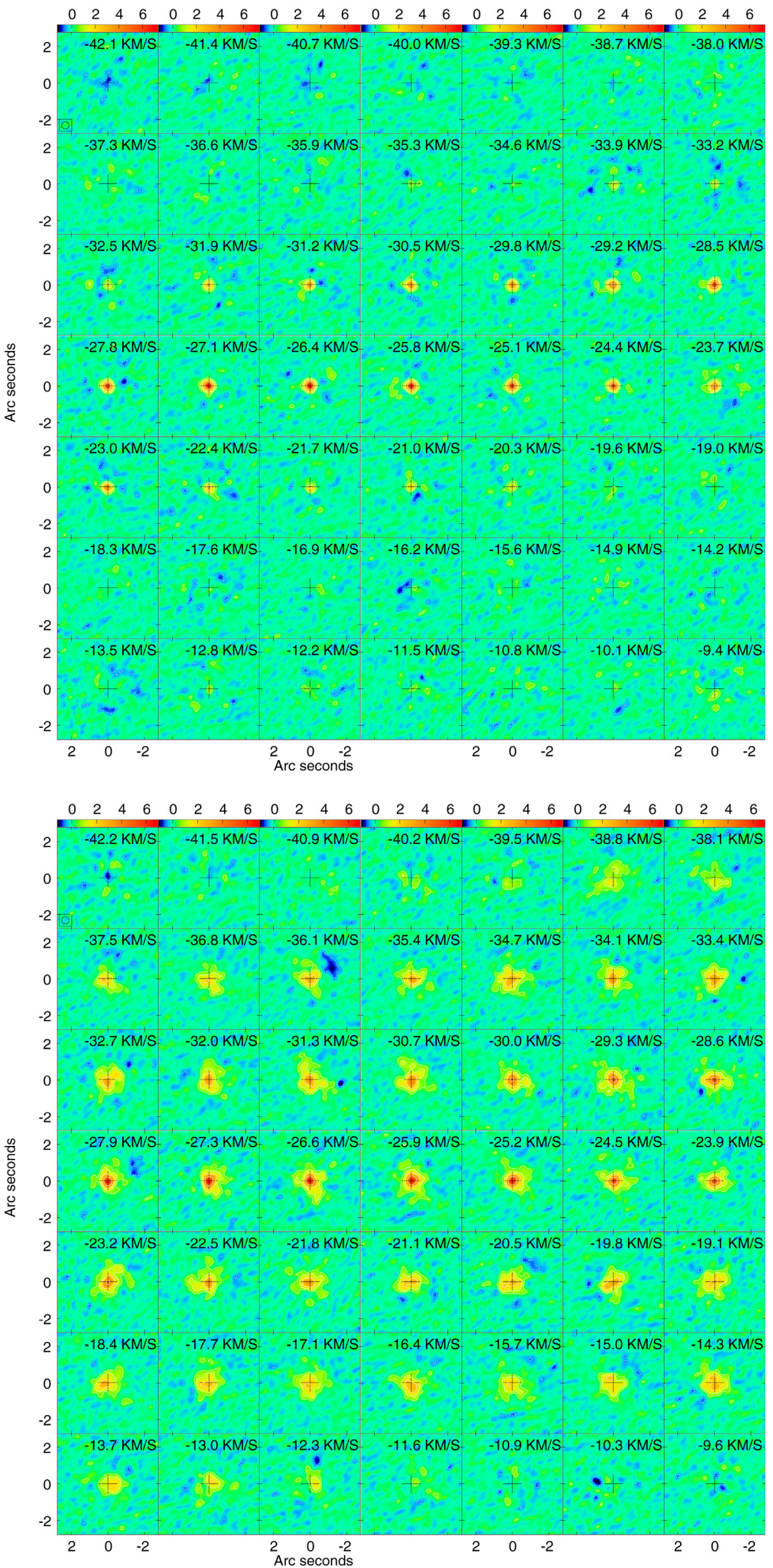

Fig. 6. $\operatorname{SiS} v=2 J=36-35$ channel map, averaged over three channels with a natural weighting and for a circular beam of $0.4^{\prime \prime}$. North is up, east is left; the black cross indicates the centre of the continuum map. The flux density units are Jy/beam. The contours are at $(-1,1$, $2,4,8 \ldots) \times 0.5 \mathrm{Jy} /$ beam $\left(\sim 3 \sigma_{\text {rms }}\right)$. This highexcitation line has a width of $\sim 9 \mathrm{~km} \mathrm{~s}^{-1}$, and the line formation region is unresolved with the current ALMA beam. The contrast in the figure is best visible on screen.
Fig. 7. $\mathrm{SiC}_{2}$ 26(4, 22)-25(4, 21) channel map, averaged over three channels with a natural weighting and for a circular beam of $0.4^{\prime \prime}$. North is up, east is left; the black cross indicates the centre of the continuum map. The flux density units are Jy/beam. The contours are at $(-1,1,2,4,8 \ldots) \times 0.5 \mathrm{Jy} /$ beam $\left(\sim 3 \sigma_{\text {rms }}\right)$. The ALMA instrument resolves the line formation region. At different velocities the flux densities display a non-spherical structure. The contrast in the figure is best visible on screen. 


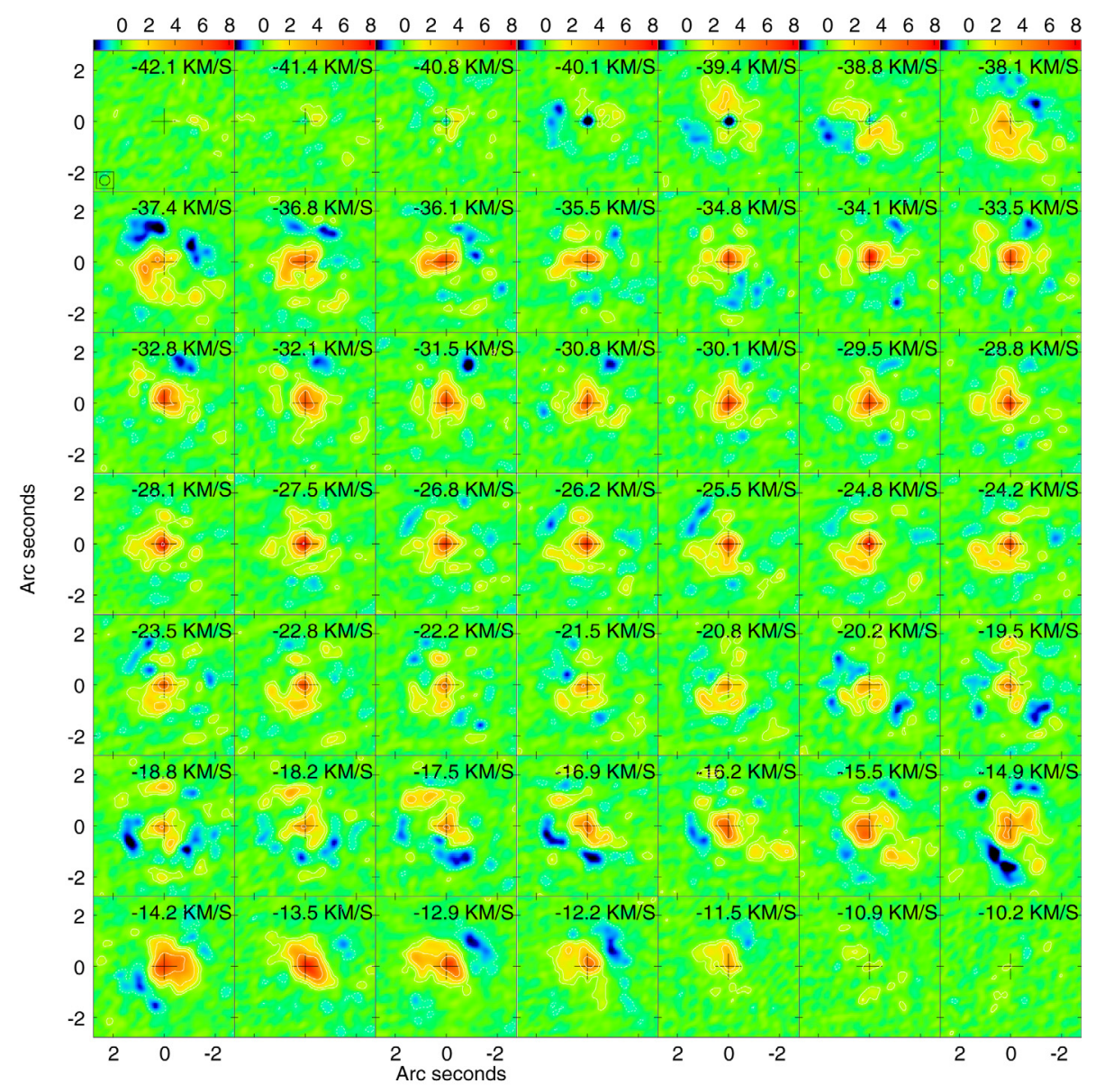

Fig. 8. ${ }^{13} \mathrm{CO} J=6-5$ channel map, averaged over three channels with a natural weighting and for a circular beam of $0.4^{\prime \prime}$. North is up, east is left; the black cross indicates the centre of the continuum map. The flux density units are $\mathrm{Jy} /$ beam. The contours are at $(-1,1,2,4$, $8 \ldots) \times 0.5 \mathrm{Jy} /$ beam $\left(\sim 3 \sigma_{\text {rms }}\right)$. Different arclike features can be discerned. The contrast in the figure is best visible on screen. milli-arcsec (see Table 1), and these lines are formed in a region of the wind where the velocity has already reached the terminal velocity.

The ${ }^{13} \mathrm{CO} J=6-5$ channel map (Fig. 8) displays a complex structure. At different velocities, parts of a spirallike arm (arcs) can be distinguished: around $-17.5 \mathrm{~km} \mathrm{~s}^{-1}$ an arc extends to the north at $\sim 1.5^{\prime \prime}$ offset from the central source, around $-24.6 \mathrm{~km} \mathrm{~s}^{-1}$ at $0.7^{\prime \prime}$ offset to the southeast, around $-27.5 \mathrm{~km} \mathrm{~s}^{-1}$ at $1^{\prime \prime}$ to the south-west, and around $-37.4 \mathrm{~km} \mathrm{~s}^{-1}$ at $2^{\prime \prime}$ to the south-west. Local density enhancements, the change in (projected) velocity for different arcs, gaps in the UV-coverage (see Sect. 2.2) and the fact that we resolve out flux for scales larger than $\sim 3^{\prime \prime}$ can explain why the spiral arms are not complete. For example, at $-37.4 \mathrm{~km} \mathrm{~s}^{-1}$, the extended emission has a distinctive curve that is unrelated to the beam sidelobe pattern. The negative artefact is due to the "bowl" effect mentioned in Sect. 2.2, resulting from emission on scales just larger than are sampled by the interferometer. Although the detailed flux distribution at radii $\gtrsim 11^{\prime \prime} 5$ is unreliable, it does represent the general shape of the brighter emission at each velocity.

The local density enhancements or spiral arcs are seen best in the maps of ${ }^{13} \mathrm{CO}$ because this is the lowest-excitation and most abundant transition in our ALMA observations, mitigating the over-resolution of its extended emission. Some of the lessabundant, intermediate-excitation lines have a similar LAS for integrated emission in the ALMA data, but are too faint to resolve extended details, spectrally or spatially. Figure 9 compares the integrated ${ }^{13} \mathrm{CO} J=6-5$ emission (zeroth moment) and dust emission. Bright arcs at $1^{\prime \prime}$ to the north and $1^{\prime \prime}$ and $1.6^{\prime \prime}$ to the south-west can clearly be discerned in the ${ }^{13} \mathrm{CO}$ emission, but the dust emission at $650 \mathrm{GHz}$ is below our detection threshold at

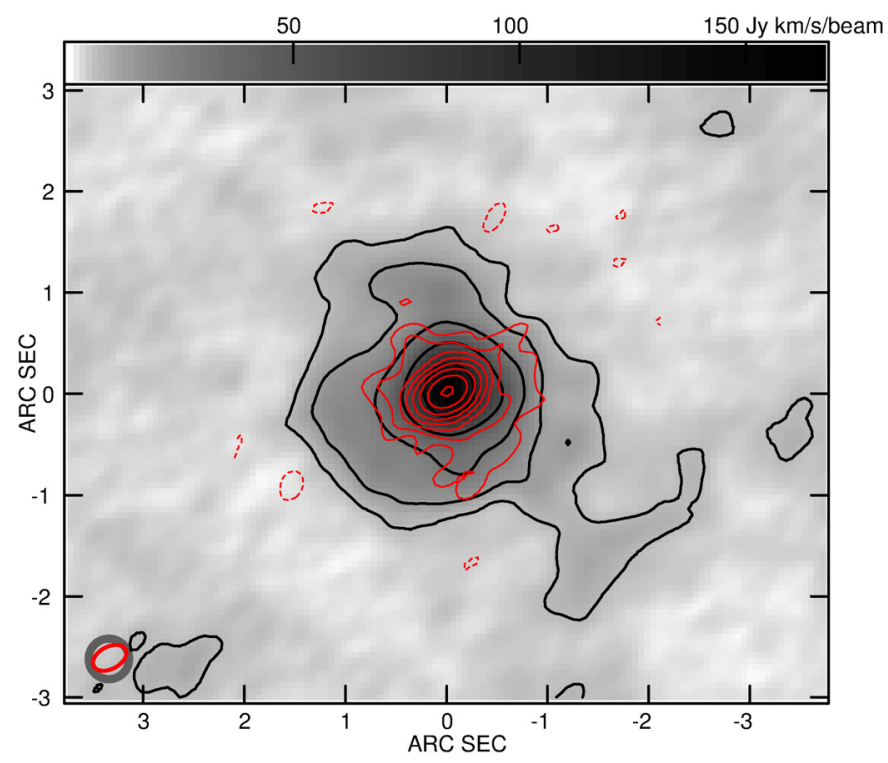

Fig. 9. Gray scale: integrated ${ }^{13} \mathrm{CO} J=6-5$ emission (cut-off level of $0.02 \mathrm{Jy} /$ beam), with contour levels at $[-1,1,2,4,8,16] \times$ $6.82 \mathrm{Jy} \cdot \mathrm{km} \mathrm{s}^{-1}$ per beam. Red contours: dust emission with contours levels are at $[-1,1,2,3,4,8,16,28] \times 15 \mathrm{mJy}^{-1}$ beam ${ }^{-1}$ (cf. Fig. 2). In the bottom left corner, the beam ellipse for the integrated ${ }^{13} \mathrm{CO} J=6-5$ emission is shown in grey and for the dust continuum in red.

radii beyond $1^{\prime \prime}$. This is the first time that we can confirm that the extended continuum and molecular emission are both centred on the continuum peak position. 

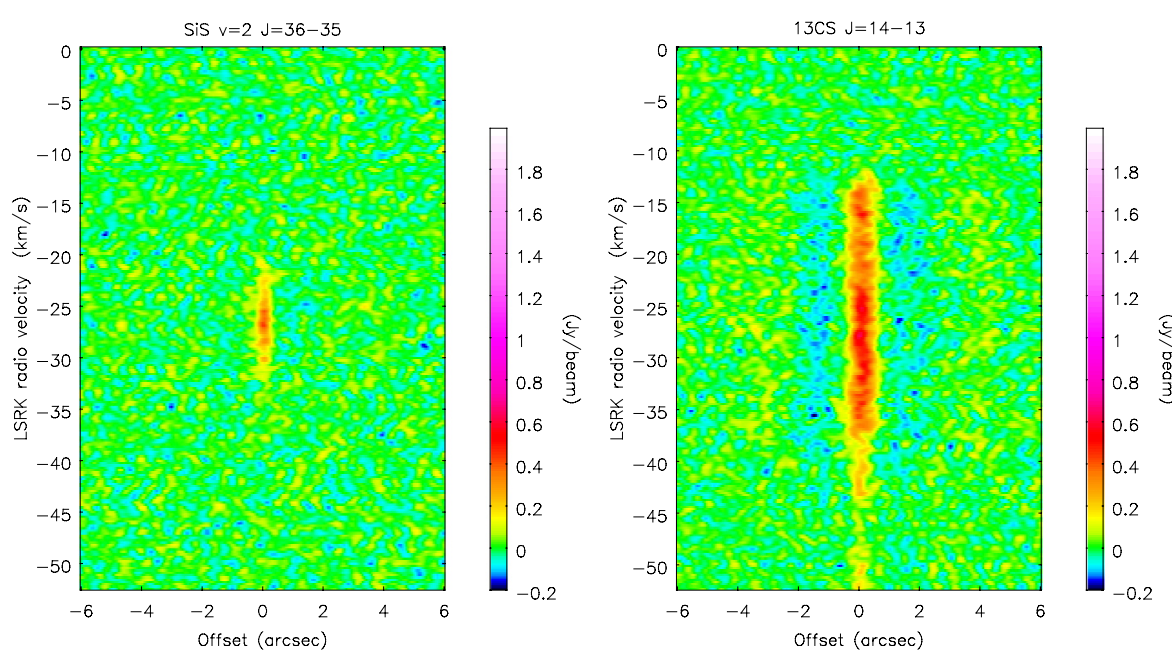

Fig. 10. Position-velocity diagram in right ascension for $\operatorname{SiS} v=2 J=36-35$ at $646.100 \mathrm{GHz}$ (left) and ${ }^{13} \mathrm{CS} J=14-13$ at $647.076 \mathrm{GHz}$ (right). Note that an offset to the east is negative, and to the west positive. Sidelobe-effects are seen in the right image, as well as the fact that the ${ }^{13} \mathrm{CS} J=14-13$ line is blended in the blue wing with a "U"-line.

\subsection{Position-velocity diagrams and morphology}

Using the CASA task IMPV, position-velocity diagrams were calculated for all transitions. In the standard set-up, 201 slits with a width of 1 pixel $\left(=0.060^{\prime \prime}\right)$ and a length of 101 pixels $\left(=6.06^{\prime \prime}\right)$ were taken. Each slit was then centred perpendicular to the direction slice (i.e. in this case 3.03" above and 3.03" below the central pixel), and the average for each slit was taken. A PV along the right ascension axis hence has $\mathrm{PA}=90^{\circ}$, and along the declination axis $\mathrm{PA}=0^{\circ}$. The strength of PV plots is that they correlate structure at different spatial offsets, which is otherwise a challenging task from channel maps alone.

As discussed in Sect. 2.2, the PV diagrams are not free from artefacts. (1) For lines with an integrated line peak flux higher than $0.4 \mathrm{Jy} / \mathrm{beam}$, sidelobe effects are clearly visible at $-4^{\prime \prime}$ and $+4^{\prime \prime}$ offset (see right panel in Fig. 10). (2) The instrument resolves out some flux for most of the lines, which is especially true for the emission of the $\mathrm{CO}$ isotopologues (see Sect. 4.2). In some PV diagrams, emission extends beyond the -10 to $-40 \mathrm{~km} \mathrm{~s}^{-1}$ range (covering the radially outflowing wind), which is due to blending with molecular line transitions at slightly different frequencies (see right panel in Fig. 10).

For all transitions, the channel maps and PV diagrams indicate that (some part of) the emission originates from the region just above the stellar photosphere, that is, within $\sim 0.2^{\prime \prime}$ or $10 R_{\star}$ (see Appendix A). The ALMA data prove that $\mathrm{SiO}$ is formed in the inner wind region, indicating an active photochemical region where shock-driven chemistry (Cherchneff 2011) and/or photodissociation of molecules in a clumpy medium (Agúndez et al. 2010) dictate the chemical balance.

Figure 11 shows the PV diagrams of the ${ }^{13} \mathrm{CO} J=6-5$ channel maps for a direction slice in right ascension or in declination. These PV diagrams display a more complex morphokinematical structure than the PV diagrams in Fig. 10, or any other line. This is expected because the ${ }^{13} \mathrm{CO} J=6-5$ line is the strongest in the frequency range we covered, as discussed in Sect 5.1. It is clear that the curved distribution of the ${ }^{13} \mathrm{CO} \mathrm{PV}$ maps represents the genuine morpho-kinematical shape, but that the actual distribution of the emission on scales larger than $\sim 3^{\prime \prime}$ is artificially fragmented as a result of the gaps in UV spacing (see Sect. 2.2). By changing the position angle of the PV diagram, changes in the morpho-kinematical structure can be diagnosed. The most revealing ${ }^{13} \mathrm{CO} J=6-5 \mathrm{PV}$-diagram is obtained for position angles around $10-25^{\circ}$ to the northeast (see Fig. 11). To show and enhance the effect of the small-scale structure, we also calculated these PV diagrams for a slit of 51 pixels (instead of 101 pixels in the standard setup); see bottom panels in Fig. 12. In all panels of Fig. 12, one can clearly distinguish correlated curved structures ranging from -40 to $-12 \mathrm{~km} \mathrm{~s}^{-1}$ and the distribution of the brightest emission around 0 " offset displays an " $S$ "-shape.

\section{Qualitative interpretation of the ${ }^{13} \mathrm{CO} J=6-5$ ALMA data}

The ${ }^{13} \mathrm{CO} J=6-5 \mathrm{PV}$ diagrams in Fig. 12 show a very characteristic shape with correlated structure at an interval of $\sim 1^{\prime \prime}$ (around the systemic velocity) for position angles of about $10-20^{\circ}$. These structures are almost absent from the PV diagrams for PA $\sim 112.5^{\circ}$. In almost all PV diagrams a typical " $S$ "-shape is seen around zero offset. Based on the simulations of Kim et al. (2013), we postulate that this type of morphology implies a spiral structure induced by a binary companion. In contrast to R Scl, for which ALMA data have recently shown a spiral seen almost face-on (Maercker et al. 2012), ALMA data of CW Leo are reminiscent of a spiral seen almost edge-on, with the orbital axis at an angle of $\sim 22.5^{\circ}$ north-east (see Sect. 8.1). Our ALMA images capture fragments of the spiral arms, seen almost edge-on, with a typical width of $\sim 300$ mas. We cannot be sure whether the fragmentation is an instrumental artefact, but for CIT 6 the 0.'7-resolution data analysed by Kim et al. (2013) show that the structure in the spiral arms is rough. High spatial resolution observations (Tuthill et al. 2000; Weigelt et al. 2002; Menut et al. 2007) and hydrodynamical models (Woitke 2006) have shown that gas and dust clumps are created in the inner envelope by the wind formation mechanism. These density inhomogeneities will remain in the inner spiral-arm structures until they are eventually dispersed.

No direct evidence has yet been found for a binary companion. However, the current ALMA data in combination with a plethora of other observational diagnostics (schematized in Fig. 13) suggest that CW Leo is part of a binary system. (1) Guélin et al. (1993) suggested that CW Leo is a binary since the clumpy shells observed in $\mathrm{MgNC}, \mathrm{C}_{4} \mathrm{H}$, and other carbonchain molecules and radicals, appear off-centre from the continuum source by $2-3^{\prime \prime}$ east. The cause of such a drift could be an acceleration of the star due to a companion. (2) As mentioned in Sect. 1, the central regions around CW Leo show an axisymmetric peanut- or bipolar-like structure with the major axis 

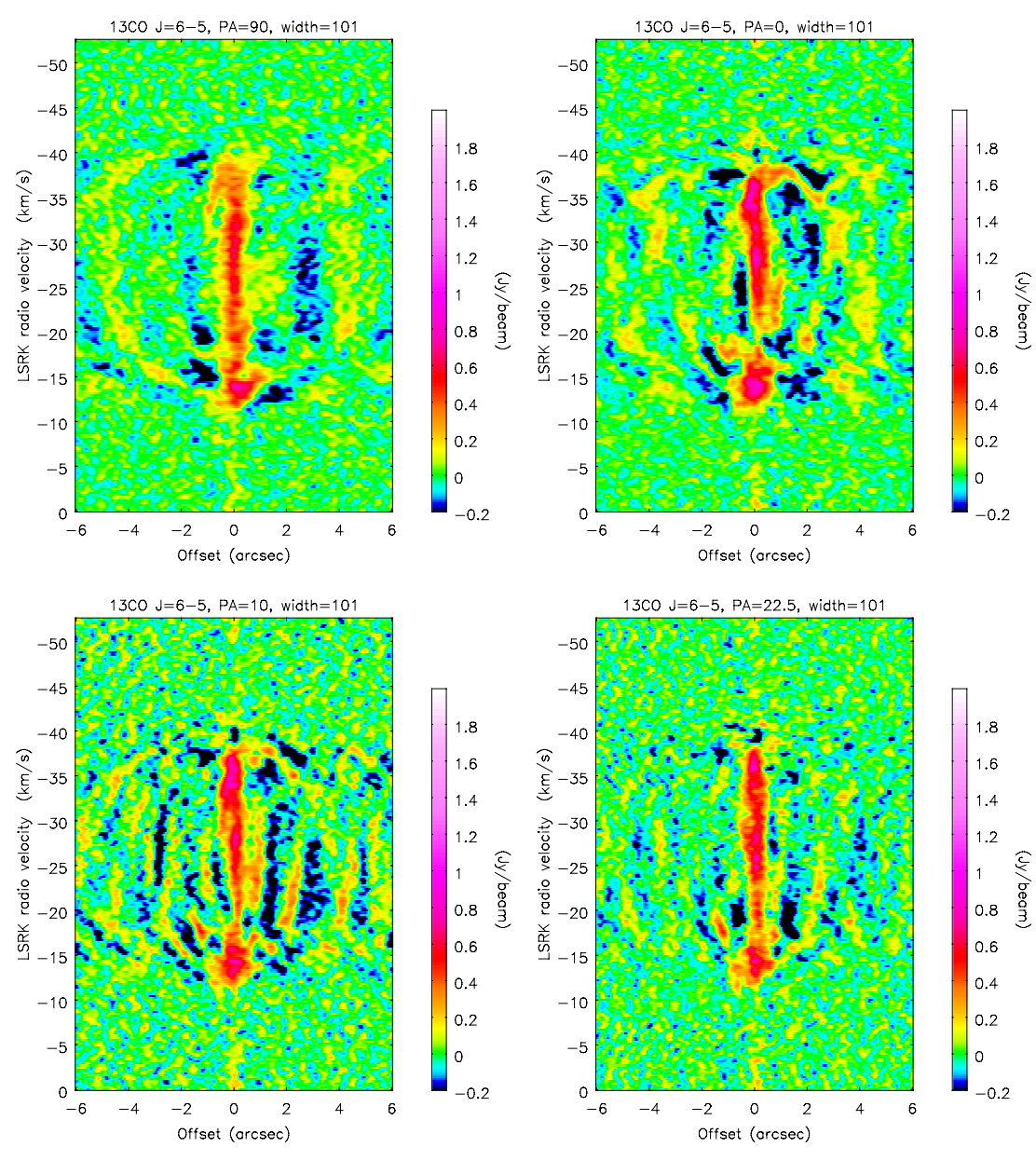

Fig. 11. Position-velocity diagram of ${ }^{13} \mathrm{CO}$ $J=6-5$ at $661.067 \mathrm{GHz}$ for a slit width of 101 pixels at a position angle (PA, measured from north to east) of (top left) $90^{\circ}$ (i.e., along the right ascension; offset to the east is negative, and to the west positive), (top right) $0^{\circ}$ (i.e., along the declination; offset to the north is negative and to the south positive), (bottom left) $10^{\circ}$, and (bottom right) $22.5^{\circ}$.

lying at a position angle of $\sim 8-22^{\circ}$ (see Fig. 13; Le Bertre et al. 1989; Skinner et al. 1998; Mauron \& Huggins 2000; Kastner \& Weintraub 1994). This is indirect evidence of a possible equatorial density enhancement of dust and gas, with the equatorial plane being located perpendicular to the major axis of the nebula, enhancing scattered stellar light emission through the biconical openings. A direct detection of an equatorial dust lane seen almost edge-on was recently presented by Jeffers et al. (2014), corroborating the results of Murakawa et al. (2005). The inferred radius of the dust lane is $\sim 0.5-1^{\prime \prime}$. The elongated east-west structure seen in our ALMA continuum images at a PA of $\sim 128^{\circ}$ with a length of 1.8" across (Figs. 2 and 9) might be the signature of this equatorial density enhancement ${ }^{5}$, while the extended emission at a PA of $\sim 20^{\circ}$ and $\sim 200^{\circ}$ might reflect the biconical cones. This type of morphology can be explained in terms of a binary system in which angular momentum provides a natural way to define the equatorial plane and polar axis. Interaction between the secondary and expanding red giant primary star will create a bipolar nebula, and the dissipative tidal interaction might eventually lead to a merger of the two stars in a common-envelope system (Morris 1987). (3) Optical and infrared data proved that there are multiple almost concentric shells (or arcs) out to $320^{\prime \prime}$ from the central star (Mauron \& Huggins 1999; Leão et al. 2006; Fong et al. 2003; Decin et al. 2011). The shells are incomplete and cover $\sim 30^{\circ}-90^{\circ}$ in azimuth. These shells might represent the limb-brightened spiral arms. The shell-intershell

\footnotetext{
5 Although we note that this direction is close to the direction of elongation of the natural synthesised beam and may be coincidence (see Sect 3.2).
}

density contrast deduced from the observations is about a factor 3-10, in agreement with recent hydrodynamical simulations for binary-induced spiral patterns as calculated by Kim \& Taam (2012c). (4) Previous observations by Guélin et al. (1993), Lucas \& Guélin (1999), and Dinh-V-Trung \& Lim (2008) of molecular shells show a lack of molecular emission at position angles of $\sim 0^{\circ}-30^{\circ}$. This position angle aligns with our inferred model rotational axis of the spiral structure (see Sect. 8.1). As shown by Mastrodemos \& Morris (1999), a binary-induced spiral structure has a lower density along the orbital axis, which might result in a lack of molecular emission along the inferred rotational axis.

Mauron \& Huggins (2000) argued against the binary scenario based on the irregular arc spacing seen in the optical and infrared images. However, the shells are projections in the plane of the sky of a complex 3D structure. A binary companion in a smooth envelope structure would indeed create a spiral structure with regular arm-spacing. However, the wind and envelope creation does not occur in a homogeneous way, adding complexity to the morpho-kinematical structure. Magnetic cool spots during the active phase (Soker \& Clayton 1999) and/or large-scale photospheric convective cells (Freytag \& Höfner 2008) can locally reduce the gas temperature, which might locally enhance the dust formation and thus lead to gas and dust clumps. The magnetic cool spots cover only a fraction of the stellar surface, and this inhomogeneity might explain the clumpiness seen on smaller spatial scales and in the $\operatorname{arcs}^{6}$. Stellar spots have not yet been observed on AGB stars, although recent observations

\footnotetext{
6 The ALMA instrumental effects also result in a superficial fragmentation of the spiral structure (see Sect. 7.2.1 and Maercker et al. 2012).
} 
L. Decin et al.: Edge-on spiral detected with ALMA in the inner wind of CW Leo

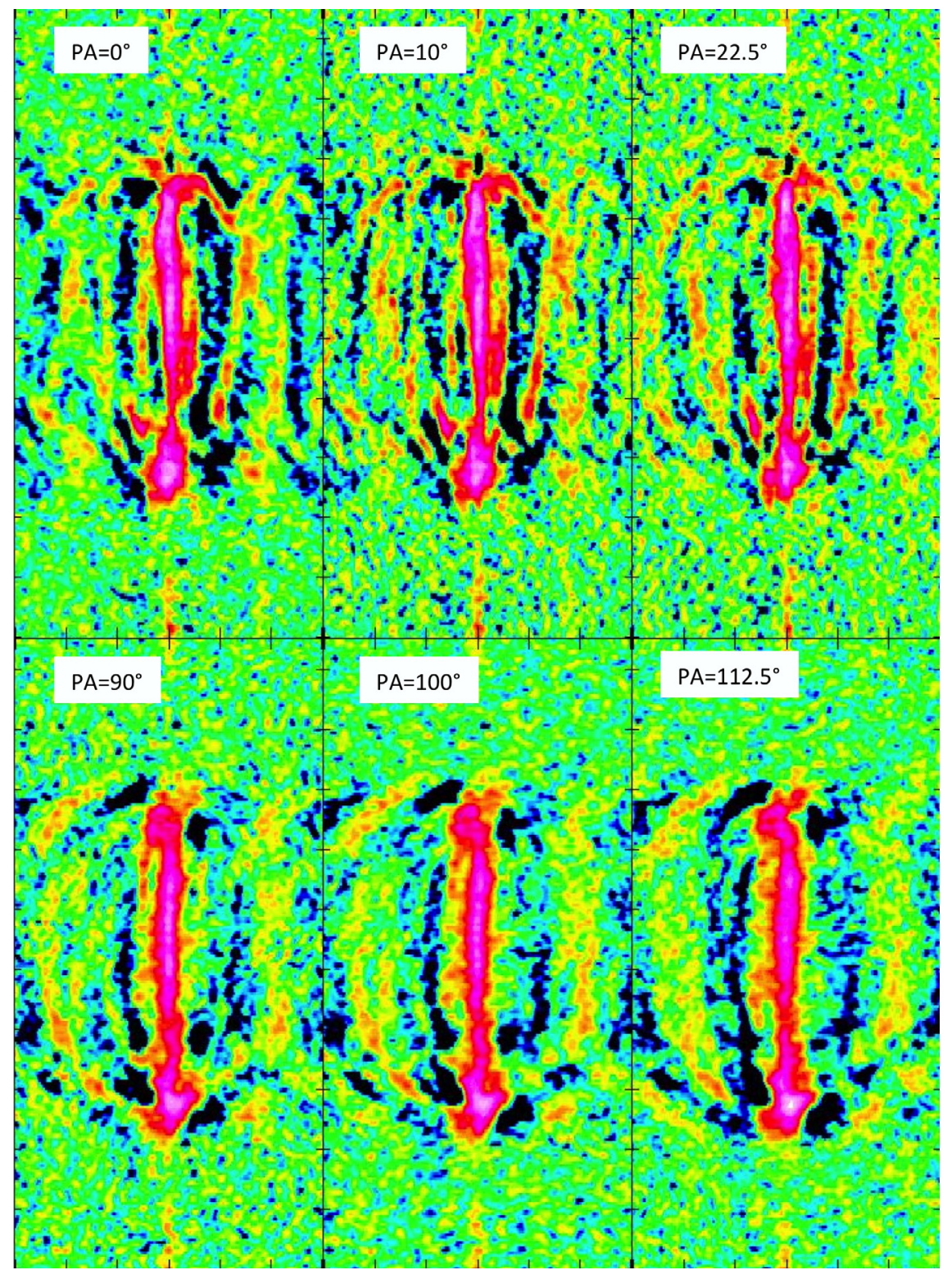

Fig. 12. Position-velocity diagram of ${ }^{13} \mathrm{CO} J=6-5$ for a slit width of 51 pixels at different position angles, indicating in the top left corner of each panel. The same color wedge has been used as in Fig. 11. The position angles at top and bottom row differ by $90^{\circ}$. The offset ( $x$-axis) ranges from $-6^{\prime \prime}$ to $6^{\prime \prime}$, the velocity ( $y$-axis) from 0 to $-55 \mathrm{~km} \mathrm{~s}^{-1}$.

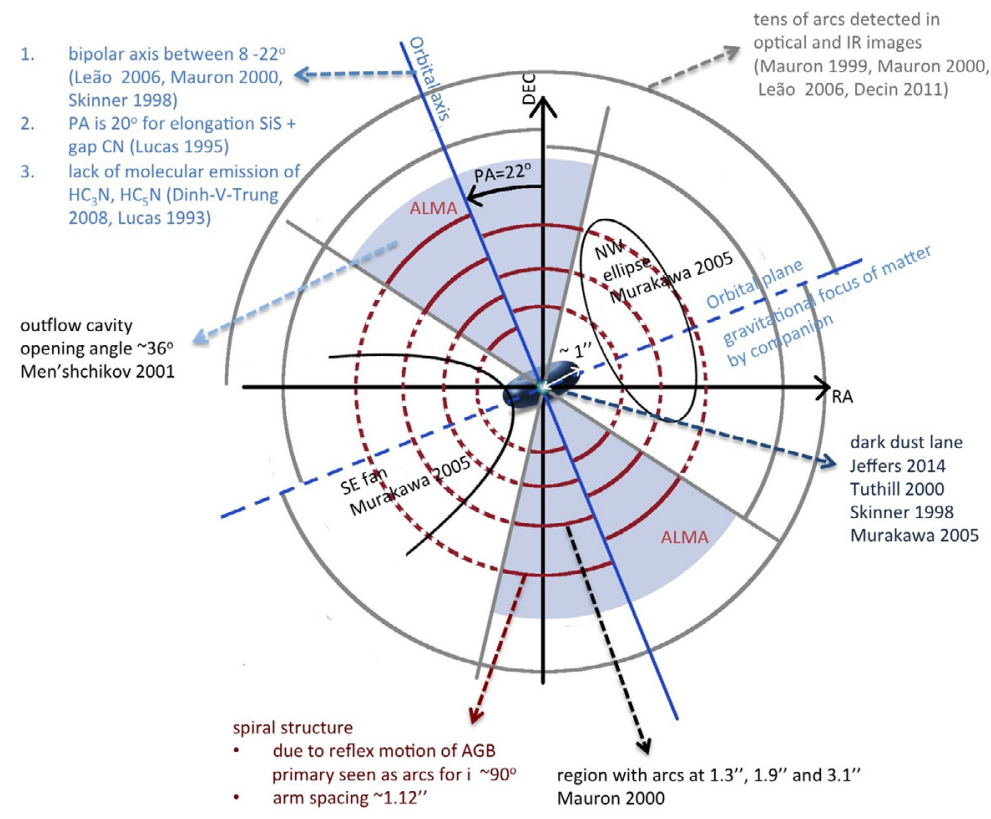

Fig. 13. Sketch of the inner wind region of CW Leo indicating the different observations and results that signal a binary companion. From the modelling of the ALMA data, we infer an orbital axis with a PA of $\sim 20^{\circ}$ (Sect. 8.1). The reflex motion of the primary AGB star results in a one-armed spiral structure that is seen almost edge-on and with an extent almost reaching the orbital axis (full red arcs). The dust lane with a radius of $\sim 0.5-1^{\prime \prime}$ situated in the orbital plane partly impedes the expansion of the spiral shock in the orbital plane direction (illustrated by the dashed red arcs). 
of linear polarization in CO $J=3-2$, SiS $J=19-18$ and CS $J=7-6$ suggest a complex magnetic field configuration in the wind of CW Leo with a strength of about $50-300 \mathrm{mG}$ at $\sim 3$ " offset of the central source (Girart et al. 2012), and recent interferometric data show strong evidence for inhomogeneities in the molecular photospheric layers of AGB stars (Wittkowski et al. 2011). In addition, modulations in the density structure might be a natural outcome from the dust formation process during which a complex non-linear interplay between gas-grain drift, grain nucleation, radiation pressure, and envelope hydrodynamics create density irregularities. Moreover, flow instabilities (such as Rayleigh-Taylor instabilities) have time to fragment the outward-moving density structures and can produce numerous small-scale cloud-like sub-structures (Woitke 2006).

\section{Modelling a binary-induced spiral structure}

A full reconstruction of the intensities, the 3D shell pattern and its kinematics using the ALMA ${ }^{13} \mathrm{CO}$ channel maps and PV diagrams requires detailed hydrodynamical simulations and radiative transfer calculations. This is beyond the scope of the current paper, but will be presented in a forthcoming paper (Homan et al., in prep.). In this section, we wish to demonstrate how different density fluctuations affect a PV diagram and how the morphology in the current ALMA PV data implies the influence of a binary companion in the shaping of the wind structure around CW Leo. The simulations were obtained using the SHAPE software developed by Steffen \& López (2006) and Steffen et al. (2011).

In this section, we first briefly describe the effect of a binary companion on the wind envelope structure (Sect. 7.1). Then, we present SHAPE simulations, where we gradually increased the complexity of the envelope structure (Sect. 7.2). In Sect. 8.1, we demonstrate the similarities between the ALMA ${ }^{13} \mathrm{CO}$ PV diagrams and PV diagrams based on spiral structures.

\subsection{Binary-induced spiral structures}

As shown in the seminal work of Mastrodemos \& Morris (1999), a binary companion might produce a spiral pattern in the circumstellar wind material. Kim \& Taam (2012b) and Kim \& Taam (2012c) have studied the separate effects of the orbital motions of the individual stars and have shown that two types of spiral patterns are created. Firstly, a more flattened spiral pattern confined within a very limited height from the orbital plane is created by the companion's motion (Kim \& Taam 2012b). Secondly, a spiral-shell-shaped pattern is created by the orbital motion of the mass-losing star around the centre of gravity (Kim $\&$ Taam 2012c). These two spiral patterns are different in several ways. (1) The companion's wake is attached to the companion, while the pattern due to the motion of the mass-losing star has a stand-off radius defined by the orbital and wind velocity (see Eq. (7) in Kim \& Taam 2012c). (2) Secondly, the propagation speeds of the patterns determining the shape are different. However, for AGB stars, this difference is very small since the propagation speed of all patterns is close to the wind speed, which dominates the orbital and sound speed. (3) Thirdly, while the arc pattern due to the reflex motion of the mass-losing star nearly reaches the orbital axis and introduces an oblate-shaped flattening of the circumstellar envelope density, the direct effect of the companion results in a spiral structure confined toward the orbital plane. When combining both types of spiral structures, the hydrodynamical models show clumpy structures within the vertical extension limit of the companion's wake as a result of shocks.

Kim et al. (2013) presented PV diagrams at different inclinations for simulations including both types of spirals for the carbon star CIT 6. Note that a spherical central region of $2^{\prime \prime}$ was carved out in their simulations to mimic the central hole seen in the observed $\mathrm{HC}_{3} \mathrm{~N}$ data. The PV diagrams in their simulations are dominated by the spiral induced by the reflex motion. In Sect. 7.2, we show that for spiral structures quite confined towards the orbital plane and for position angles significantly different from the orbital axis or plane, an $S$-type feature around offset zero appears in a PV diagram around offset zero when seen almost edge-on; this feature is also seen in the ALMA data (see Fig. 11).

\subsection{Morpho-kinematical simulations}

To understand the complex morpho-kinematical structure seen in the ${ }^{13} \mathrm{CO} \mathrm{PV}$ diagrams and link the ALMA data to a 3D shell pattern, we used the SHAPE modelling tool (Steffen \& López 2006; Steffen et al. 2011). This is a flexible interactive 3D morpho-kinematical modelling application for astrophysics that is publicly available. By interactively defining 3D structures, it calculates intensity maps, PV diagrams, channel maps, and spectra. While eulerian 3D grid-based hydrodynamic simulations are possible, in this work we used a purely mathematical description of the object structure (see below). A 3D mesh was constructed that served as a container of the emissivity and velocity field. If the radiation transfer is computed, the information in the mesh is transferred to a regular Cartesian 3D grid, which is used to compute the radiation transfer. The advantage of SHAPE is that it is computationally very fast, which facilitates a first broad screening of the huge 3D kinematical and morphological parameter space. The deduced model parameters can then be used as input for a more detailed (hydrodynamical) simulation. Recently, a non-local-thermodynamic equilibrium (non-LTE) radiative transfer solver (SHAPEMOL) has been added to compute molecular excitation levels (Santander-García et al. 2012, 2015). This solver is based on the well-known LVG (Large Velocity Gradient, Castor 1970) approximation, which significantly simplifies the radiative transfer problem. The current version of SHAPEMOL still has limitations when calculating accurately the molecular level populations in an AGB wind: it only includes collisional rate constants for temperatures up to $1000 \mathrm{~K}$ and $\mathrm{H}_{2}$ densities between $1 \times 10^{13}$ to $1 \times 10^{8} \mathrm{~m}^{-3}$, rotational levels in the vibrational excited states are not included, and the tabulated densities are sometimes too low for the high densities encountered in the inner wind ${ }^{7}$. Nonetheless, it can still be used for a first morpho-kinematical interpretation of the structure seen in the ALMA PV data of ${ }^{13} \mathrm{CO}$.

Based on the results of Decin et al. (2010b) and De Beck et al. (2012), we chose as input for our basic models a distance of $150 \mathrm{pc}$, a constant gas mass-loss rate of $1.5 \times 10^{-5} M_{\odot} / \mathrm{yr}$, a fractional abundance $\left[\mathrm{CO} / \mathrm{H}_{2}\right]$ of $6 \times 10^{-4}$, an isotopologue ratio ${ }^{12} \mathrm{CO} /{ }^{13} \mathrm{CO}$ of 30 , a stellar radius of $4.55 \times 10^{13} \mathrm{~cm}$ (or $0.02^{\prime \prime}$

7 Tabulated $\mathrm{H}_{2}$ densities in SHAPEMOL range from $1 \times 10^{13}$ to $1 \times 10^{8} \mathrm{~m}^{-3}$, while the wind of $\mathrm{CW}$ Leo has a $\mathrm{H}_{2}$ density of about $1 \times 10^{15} \mathrm{~m}^{-3}$ at $2.5 R_{\star}$ (or $0^{\prime} .05$ ), reaching $6.6 \times 10^{10} \mathrm{~m}^{-3}$ at $6^{\prime \prime}$. Since the interest of this paper is not to reproduce the ${ }^{13} \mathrm{CO}$ intensity maps but to analyse the morpho-kinematical structure in the inner envelope, we divided the overall density by a factor 100 . 

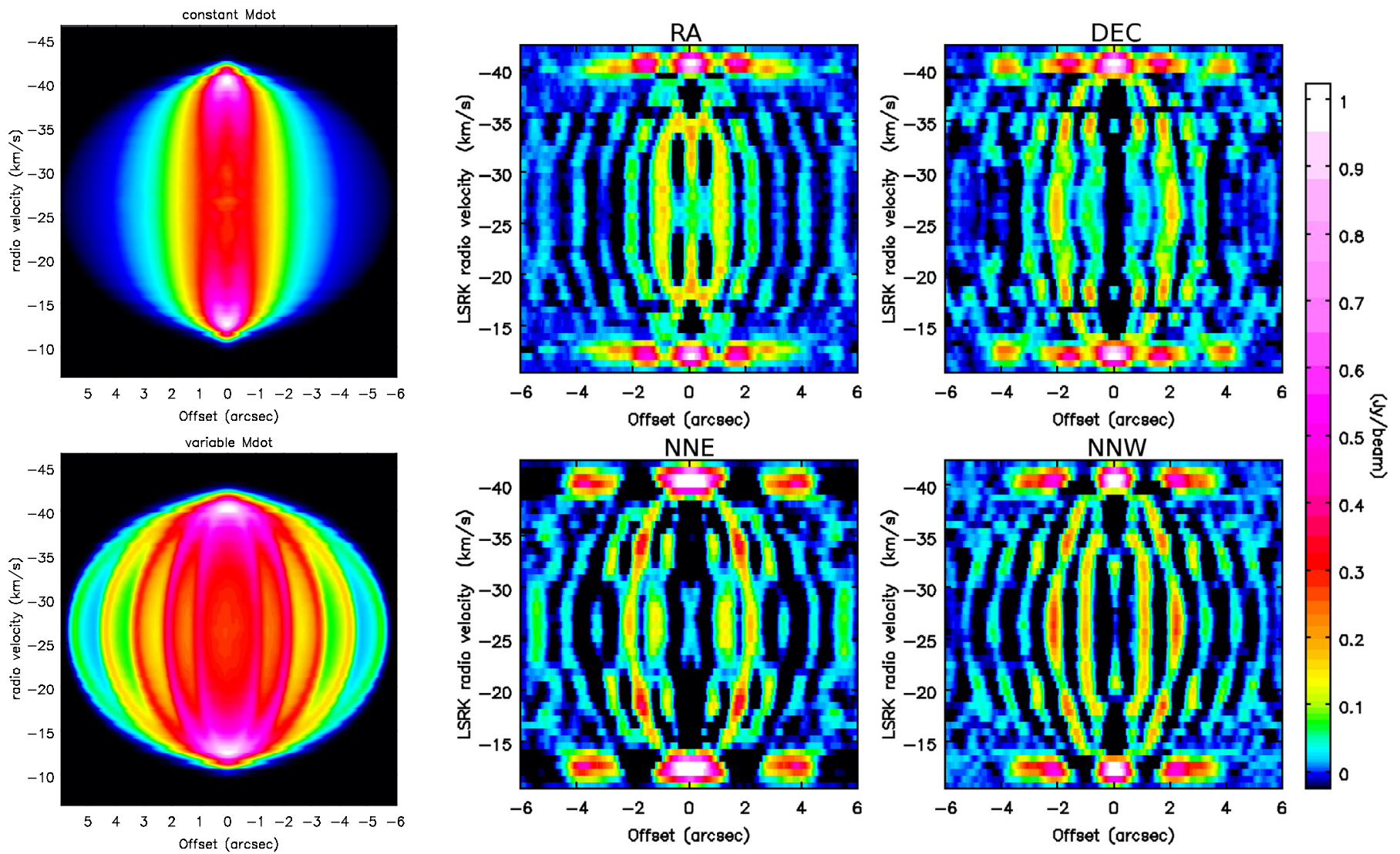

Fig. 14. Simulated ${ }^{13} \mathrm{CO} J=6-5 \mathrm{PV}$ diagrams for a beam of $0.43 \times 0$ ' 23 . Top left: model for radially outflowing wind for a constant mass-loss rate $\left(\rho \propto r^{-2}\right)$. Bottom left: model for a radial outflowing wind with varying wind density, with the shell-intershell density contrast being 10 . The density-enhanced shells are placed with an interval of $1^{\prime \prime}$. No instrumental effects were taken into account. Right: simulations for the PV-diagram for the density-enhanced shells taking the ALMA instrumental effects into account: in the four sub-panels, the slit is varied to create a PV diagram along the right ascension coordinate, the declination coordinate, and $22.5^{\circ}$ to the north-east (NNE) or to the north-west (NNW).

at $150 \mathrm{pc}$ ), and a wind velocity of $14.5 \mathrm{~km} \mathrm{~s}^{-1}$. The gas kinetic temperature is assumed to follow a power law

$T(r)=T_{\mathrm{eff}}\left(\frac{R_{\star}}{r}\right)^{\zeta}$,

with $T_{\text {eff }}$ the effective temperature of $2330 \mathrm{~K}$ and $\zeta \approx 0.5$. The molecular line data (energy levels, Einstein A coefficients and collisional rates) were taken from the LAMDA database (Schöier et al. 2005).

We produced models of the inner 6" (i.e., total width of $12^{\prime \prime}$ ), at $\sim 47$ mas resolution for spectral channels separated by $0.4 \mathrm{~km} \mathrm{~s}^{-1}$. We simulated the effect of the ALMA setup using the CASA task SIMOBSERVE, with antenna positions and atmospheric conditions matching those used for our observations. To obtain the same total duration (and range of $u v$ spacings sampled), the simulations had a slightly longer on-target duration. The simulated model images and PV plots therefore provide sampling, including missing spacings, similar to the original observations. The noise in the simulated images is slightly lower, not only because of the longer time on-source, but because although atmospheric and instrumental noise affecting the target are included, the limitations due to other imperfections in phase calibration are not. The plots of simulated data do have similar dynamic range limitations and artefacts due to missing flux (where the input model has a large-scale structure) as the observed images. For example, the absolute value of the most negative emission in the simulated data in the right panels of Fig. 14 is $\sim 30 \%$ of the peak. The look-up tables in some plots were curtailed to highlight the most relevant features for comparison. We used the same CLEAN parameters as for our observations to produce image cubes and position-velocity plots.

\subsubsection{PV diagram for a radially outflowing wind}

We first simulated two simple examples for a radially outflowing wind at a constant expansion velocity of $14.5 \mathrm{~km} \mathrm{~s}^{-1}$. In the first case, the mass-loss rate was assumed to be constant (or density $\rho \propto r^{-2}$; see top left panel in Fig. 14); while in the second example (bottom left and right panels in Fig. 14) we demonstrate the effect of density-enhanced shells (Cordiner \& Millar 2009; Decin et al. 2011) with a shell-intershell density contrast of a factor 10. The wind region close to the stellar surface is responsible for the bright bar at zero offset in the PV diagrams, while the outer density-enhanced shells create well-distinguished correlated curved structures at larger offset in the PV diagram. Including the ALMA instrumental effects results in a (virtual) fragmentation of the shells (see right panels in Fig. 14). Owing to the short hour-angle coverage of the simulated observations in Fig. 14 (right panels), the simulated PV diagrams at different position angles are not identical ${ }^{8}$.

The simulated PV diagrams for the density-enhanced shells taking the ALMA instrumental effects into account show some

\footnotetext{
8 Note that for the real ALMA observations this effect is not so strong since they were spread out more in hour-angle because of the longer gaps between scans spent on the calibration sources.
} 
resemblance with the ALMA ${ }^{13} \mathrm{CO} J=6-5 \mathrm{PV}$ diagram for a position angle around $22^{\circ}$ north-east (Fig. 12). However, a spherical symmetric geometry and isotropic density wind structure as applied in the current setup cannot explain the different morphologies seen in the PV diagram with position angles $90^{\circ}$ apart from each other and the asymmetry when reflecting around zerooffset (the " $S$ "-shape as seen, e.g., for a $\mathrm{PA}=0^{\circ}$ and $\mathrm{PA}=90^{\circ}$ in Fig. 12).

\subsubsection{PV diagram for binary-induced spiral wind structure}

In this section, we simulate the effect of a spiral structure on the PV diagram. Both the spiral structure due to the companion's wake and that due to the reflex motion of the mass-losing AGB star are simulated using a simple analytical description. To do so, we followed the results described by Kim \& Taam (2012b) and Kim \& Taam (2012c) by defining an Archimedean spiral $r=a_{\mathrm{p}} \theta$, with $r$ the radial coordinate, $\theta$ the longitudinal coordinate, and $a_{\mathrm{p}}$ the factor of proportionality between the angle $\theta$ and the radial coordinate $r$.

The spiral structure is generated by a thin shell mesh with its position given as the position vector $\boldsymbol{r}$ in spherical coordinates $(r, \theta, \phi)$ as a mapping of the unit sphere $(1, \theta, \phi)$, with $\phi$ the latitude ( $\phi=90^{\circ}$ being the equator). The coordinates of the mesh vertices are given by

$\boldsymbol{r}(r, \theta, \phi)=\left(a_{\mathrm{p}} \theta, \theta, \phi\right)$,

with $r$ in units of meter, $\theta$ between 0 and $360^{\circ}$, and $\phi$ between 0 and $180^{\circ}$. The spacing between two successive turnings in the plane of the sky, $a$, is proportional to $a_{\mathrm{p}}$, with $a$ defined in units of arcsec for a distance of $150 \mathrm{pc}$.

The overall radial density was taken to be $\propto r^{-p}$ (with $p \sim 2$, Kim \& Taam 2012c), which was then modified by the longitudinal and latitudinal dependencies. To simulate both types of spiral arms (confined toward the orbital plane and extending almost completely toward the orbital axis), we define the latitudinal dependence of the density as

$G(\phi)=\frac{w^{2}}{\left(\phi-90^{\circ}\right)^{2}+w^{2}}$,

in which case low values of $w$ (in units of degrees) generate a spiral arm confined toward the orbital plane. Hence the density in the spiral arms is defined as

$\rho(r, \theta, \phi)=\rho_{0} \cdot\left(\frac{r}{r_{0}}\right)^{-p} \cdot F(r, \theta) \cdot G(\phi)$

with

$F(r, \theta)=1, \quad$ inside the spiral mesh

$F(r, \theta)=0, \quad$ outside the spiral mesh,

and $\rho_{0}$ the number density at the stellar radius $\left(r_{0}\right)$ as derived from the equation of mass conservation for a mass-loss rate of $1.5 \times 10^{-5} M_{\odot} / \mathrm{yr}$. The thickness $\beta$ of the spiral arms is given as a function of distance $r$ from the centre as $\beta(r)=b r+c$, where $b$ and $c$ are parameters that determine the growth rate and the initial thickness of the spiral shell.

The different free parameters $(a, p, b, c$, inclination $i$, and $w)$ were changed to study their effect with the aim of understanding the morpho-kinematical structure visible in the ${ }^{13} \mathrm{CO} J=6-5 \mathrm{PV}$ diagrams. The inclination angle $i$ is defined as the angle between the spiral axis and the line of sight, or, equivalently, the angle between the equator and the plane of the sky.
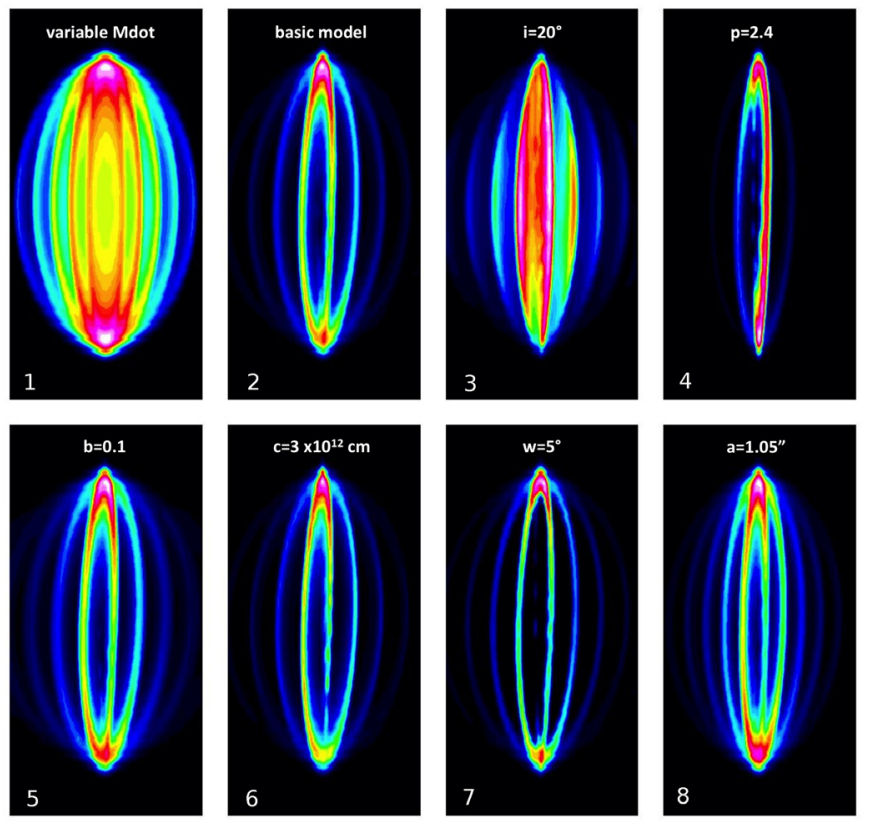

Fig. 15. ${ }^{13} \mathrm{CO} J=6-5 \mathrm{PV}$ diagram for different model setups for a spiral structure, with the $x$-axis going from -6 to $6^{\prime \prime}$ and the $y$-axis from -20 to $20 \mathrm{~km} \mathrm{~s}^{-1}$ : panel 1: radial outflowing wind with density-enhanced shells, for a shell-intershell contrast of a factor 10, panel 2: basic model for the spiral wind structure, with parameters $a=1.45, p=2, b=0$, $c=8 \times 10^{12} \mathrm{~cm}$ (or $16 R_{\star}$ ), and $w=50$ at a PA along the orbital plane, panels 3-8: same as basic model but with one parameter changed, as indicated at the top of each panel. For each panel, the colour wedge extends from zero to highest intensity (in Jy/beam), with redder colours indicating a higher intensity.

Table 3. Input values for the basic spiral model (Col. 3) and final model presented in Fig. 18.

\begin{tabular}{clcc}
\hline \hline Parameter Meaning & Value & Value \\
\hline$i$ & inclination $\left(^{\circ}\right)$ & 80 & 60 \\
$a$ & spiral arm distance $\left({ }^{\prime \prime}\right)$ & 1.45 & 1.12 \\
$p$ & power radial density profile & 2 & 2 \\
$b$ & growth rate spiral shell & 0 & 0 \\
$c$ & initial thickness spiral shell $(\mathrm{cm})$ & $8 \times 10^{12}$ & $3 \times 10^{12}$ \\
$w$ & latitudinal dependence density $\left(^{\circ}\right)$ & 50 & 30 \\
\hline
\end{tabular}

Homan et al. (in prep.) will present a much more detailed study. Although limited by the short duration of the observations, we estimate a value for the spiral-arm spacing $a$ in the range of $1-2^{\prime \prime}$ using Fig. 8.

As setup for our basic spiral model, we chose as free parameters $i=80^{\circ}$ (Jeffers et al. 2014), $a=1.45^{\prime \prime}, p=2, b=0$, $c=8 \times 10^{12} \mathrm{~cm}$ (or $16 R_{\star}$ ), and $w=50^{\circ}$ (see Table 3 ). Each of the parameters was then consecutively changed to demonstrate its effect (see Fig. 15). The PV diagram for the basic model of a one-armed spiral at a position angle along the orbital plane (second panel in Fig. 15) shows very regular structures due to the formation of higher density arcs in a plane perpendicular to the orbital plane (see also Fig. 11 in Mastrodemos \& Morris 1999), although its signature is slightly more asymmetric than the PV diagram for the radial outflowing wind with densityenhanced shells (first panel in Fig. 15). At position angles not aligned with the orbital axis or orbital plane, the difference with the radial outflowing wind is much more pronounced, as can be seen in the first panel of Fig. 16. At a PA along the orbital plane, 

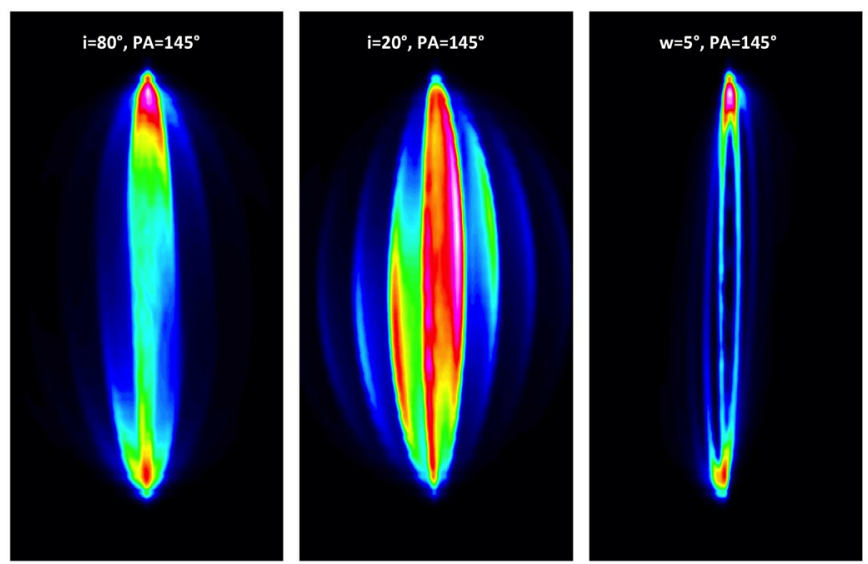

Fig. 16. ${ }^{13} \mathrm{CO} J=6-5 \mathrm{PV}$ diagram for a PA of $145^{\circ}$ for the basic model at $i=80^{\circ}$ (left), at $i=20^{\circ}$ (middle), and for $w=5^{\circ}$ (right).

the morphological difference between the models for an inclination $i=80^{\circ}$ and $i=20^{\circ}$ is mainly seen around zero offset, for which a higher intensity contrast is reached for $i=20^{\circ}$. For position angles not aligned with the orbital axis or orbital plane, a more distinct morphological difference is seen for different inclination angles (see Fig. 16): at inclination angle $\gtrsim 60^{\circ}$ an " $S$-type" structure around zero-offset is seen in the PV-diagram. This $S$-type signature is also seen in the ${ }^{13} \mathrm{CO} J=6-5$ ALMA images of CW Leo (see Fig. 11); this feature cannot be reproduced by a model assuming a radial outflowing wind with densityenhanced shells (see Fig. 14). The difference in PV diagrams for different position angles at different inclinations is used below to constrain the inclination angle of the spiral arms in CW Leo (Sect. 8.1).

As demonstrated by Kim \& Taam (2012c), the mean density in the binary-induced stellar wind is proportional to $r^{-p}$, with $p \sim 2$. Figure 15 shows the effect for increasing the value of $p$ from 2 to 2.4 (although the mass is not conserved for this latter simulation). For $p \sim 2.4$, the density is more concentrated in the inner arm spirals, clearly visible in the PV diagram.

When the parameter $b$ is larger than zero, the thickness of the spiral arms increases with radial distance from the star, simulating the effect of dispersion. This leads to a lower mean density in the outer spiral arms. In contrast, lowering the initial thickness of the spiral shells from $8 \times 10^{12} \mathrm{~cm}$ to $3 \times 10^{12} \mathrm{~cm}$ results in a higher mean density in the complete spiral structure because of the conservation of mass. Decreasing the parameter $a$ leads to a decrease in the distance between the successive arm turnings of the spiral.

A value for the parameter $w$ around 50 results in the mean density being rather uniformly distributed in latitude, while the much lower value of $w=5$ results in the latitudinal dependence of the density being more concentrated toward the orbital plane, hence mimicking the effect of gravitational focusing. While at a PA along the orbital axis the difference is not so large, the latitudinal dependence is more pronounced at $\mathrm{PA}=145^{\circ}$ (see Fig. 16).

As discussed by Mastrodemos \& Morris (1999), the effect of a binary companion is to focus some fraction of the material into a spiral structure, the remainder still forming a quasispherical envelope. This smooth wind structure is well-visible in the ALMA PV diagrams as the dominant maximum around zero spatial offset covering the full velocity range (see Fig. 17). As will be shown by Homan et al. (in prep.), the flux contrast between the correlated arcs and bright inner bar can be used to
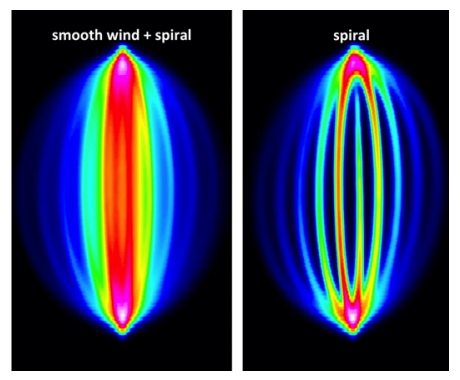

Fig. 17. Position-velocity diagram for a spiral structure with parameters $a=1.12, p=2$, $b=0, c=3 \times 10^{12} \mathrm{~cm}$, and $w=10^{\circ}$, and $i=80^{\circ}$. In the right panel only the spiral structure is modelled, while in the left panel a smooth wind is included with a density contrast of a factor 4 .

deduce the density contrast between the smooth wind and spiral structure.

\section{Discussion}

\subsection{Morphological comparison between ALMA ${ }^{13} \mathrm{CO}$ $P V$ diagrams and the theoretical simulations}

With an on-source integration time of only $17 \mathrm{~min}$, an in-depth understanding of the ${ }^{13} \mathrm{CO} J=6-5$ channel maps (Fig. 8) is challenging. However, it is clear that the different PV diagrams (Figs. 11 and 12) display correlated structures that cannot be interpreted with a simple spherical symmetric wind model. While the wind model with the density-enhanced shell structures shows some resemblance to the ALMA PV data, the asymmetry when reflecting around zero-offset seen in the ALMA PV diagrams (Fig. 12) cannot be explained by this simple model, neither can it explain the difference in the PV diagrams with $90^{\circ}$ difference in PA. A possible scenario to explain the structures seen in the ALMA PV diagrams is a binary-induced spiral structure. This interpretation is corroborated by other data and analyses already presented in the literature (see Sect. 6). Using the simple mathematical prescription for a binary-induced spiral structure described in Sect. 7.2, we derive from the ALMA PV diagrams at PA $\sim 10-20^{\circ}$ that the spiral parameter $a$ is $\sim 1.1^{\prime \prime}$. Different combinations for $p, b$, and $c$ give a good fit to the data, but the current limitations in the ALMA sensitivity of the current data and the limited UV-coverage inhibit a strict constraint. We therefore opted to use $p=2$ (mass conservation) and $b=0$ (the thickness of the spiral arms remains constant). A good fit to correlated structures in the ALMA PV data is obtained for the spiral parameters $a=1.12^{\prime \prime}, p=2, b=0, c=3 \times 10^{12} \mathrm{~cm}$, and $w=30^{\circ}$, and $i=60-80^{\circ}$ (see Fig. 18). The best constrained parameter is the spiral arm distance $a$, with an uncertainty of only $\sim 10 \%$. The general morphology is reproduced quite nicely, but the fit to the relative intensities can be improved. The latter is expected taking into account the simple approach for modelling the spiral structure. An in-depth study describing the results of a large parameter grid, including a variation in radial temperature structure of the spiral, density contrast, etc. will be presented in Homan et al. (in prep.).

It is clear that the SHAPE simulations shown in Fig. 18 do not capture the clumpiness seen in the ALMA data. Part of the fragmentation is artificial and comes from the poor UV coverage of the data (see Fig. 14). However, part of the clumpiness seems also inherent to the wind structure of CW Leo and indicates a rough gas (and dust) density distribution within the spiral arms. Magnetic cool spots, photospheric convective motions, flow instabilities, etc. may result in localized gas and dust clumps (see the discussion in Sect. 6). The fact that we see the arc structure extending toward the orbital axis (see illustration in Fig. 13) implies that the reflex motion of the primary AGB star (CW Leo 

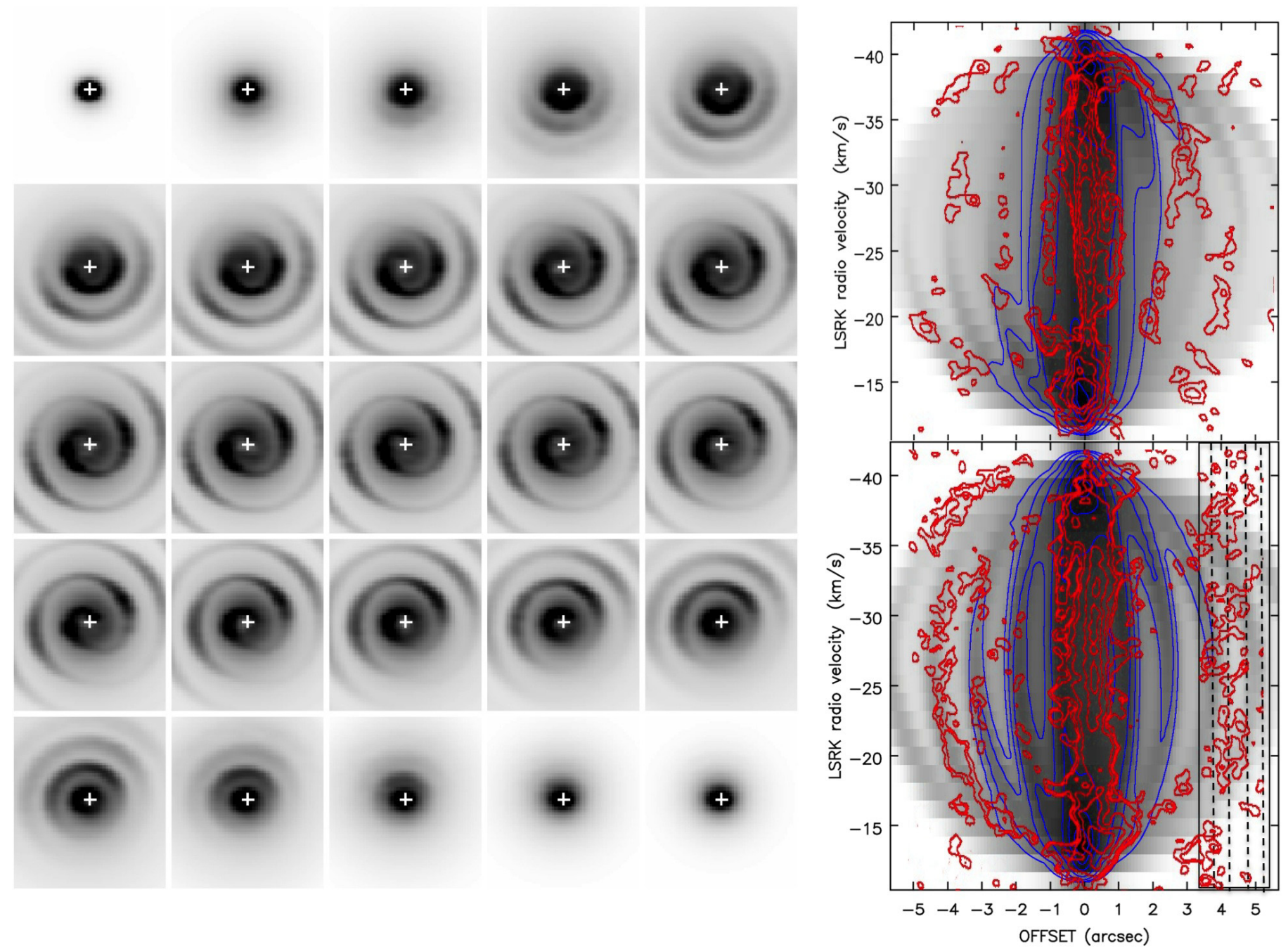

Fig. 18. Left: simulated channel maps for a spiral wind structure with parameters $a=1.12, p=2, b=0, c=3 \times 10^{12} \mathrm{~cm}, w=30^{\circ}$, and $i=60^{\circ}$, including a smooth wind structure with a spiral/wind density contrast of a factor 4 . The central velocity of the panel at the top left is $-16 \mathrm{~km} \mathrm{~s}^{-1}$ (w.r.t. $v_{\mathrm{LSR}}$ ), at the bottom right corner $+16 \mathrm{~km} \mathrm{~s}^{-1}$, hence $\Delta v$ is $1.33 \mathrm{~km} \mathrm{~s}^{-1}$ for adjacent panels. The field of view is $5^{\prime \prime} \times 5^{\prime \prime}$. The ALMA instrumental effects are not taken into account in this simulation. Right: corresponding simulated PV diagrams (in grey-scale) for a slit along the declination axis (top) and along the right ascension axis (bottom). The contours of the simulated PV diagrams are shown in blue and for the ALMA data in red, each time at $(0.05,0.1,0.2,0.4,0.6,0.8)$ times the highest intensity. The shaded region in the bottom panel indicates the place where sidelobe effects deteriorate the quality of the ALMA PV data.

itself) acts as a cause for the arc-like signature detected in the ALMA PV, Herschel, and Hubble Space Telescope images.

\subsection{Orbital period and binary separation}

The model-fitting of the ALMA ${ }^{13} \mathrm{CO} J=6-5 \mathrm{PV}$ data indicates a spiral-arm spacing of $a \sim 1.12^{\prime \prime}$, which agrees with the arc spacing seen in the HST wide- $V$ band image of the inner $10^{\prime \prime}$ region of CW Leo (see Fig. 3 in Mauron \& Huggins 2000). The arm-spacing is determined by the product of the binary orbital period $T_{\mathrm{p}}$ and the pattern propagation speed in the orbital plane, which following Kim \& Taam (2012a) is given by

$\Delta r_{\mathrm{arm}}=\left(\left\langle V_{w}\right\rangle+\frac{2}{3} V_{\mathrm{p}}\right) \times \frac{2 \pi r_{\mathrm{p}}}{V_{\mathrm{p}}}$,

with $\left\langle V_{w}\right\rangle$ the wind velocity, $V_{\mathrm{p}}$ the orbital velocity, and $r_{\mathrm{p}}$ the orbital radius of the primary, and the orbital period $T_{\mathrm{p}}$ given by the last term $\left(2 \pi r_{\mathrm{p}} / V_{\mathrm{p}}\right)$. The first term in the right-hand part of Eq. (8), $\left\langle V_{w}\right\rangle+\frac{2}{3} V_{\mathrm{p}}$, is the pattern propagation speed throughout the orbital plane, which is close to the wind speed if $V_{w}$ dominates over the orbital and sound speeds (which is the case for almost all AGB binary simulations, see Kim \& Taam 2012c, and Kim priv. comm.). At a distance of $1^{\prime \prime}$, the wind has already reached its terminal velocity of $14.5 \mathrm{~km} \mathrm{~s}^{-1}$ (see Sect. 4.3). The derived arm-spacing hence results in an orbital period of $\sim 55 \mathrm{yr}$.

The derived orbital period is lower than the orbital periods derived for some other carbon-rich AGB stars, of which a binary companion is thought to be the cause of the detected spiral arm structure. Maercker et al. (2012) derived for R Scl a period of $350 \mathrm{yr}$ on the basis of CO $J=3-2$ ALMA data, for CIT 6 Dinh-V.-Trung \& Lim (2009) found a period of $\sim 600 \mathrm{yr}$, and for AFGL 3068 an orbital period of $830 \mathrm{yr}$ was obtained by Mauron \& Huggins (2006). The longer orbital periods for these AGB stars indicate that the companion must be on a wide orbit around the AGB star; typically around 50-70 au. According to Kepler's third law and assuming a primary initial mass of $4 M_{\odot}$ for CW Leo (Guélin et al. 1995) and the mass of the secondary being lower than that of the primary, the mean binary separation, 
$d$, for CW Leo is $\sim 25 \pm 2$ au (or $\sim 8.2 R_{\star}=0.17^{\prime \prime}$ ), that is, a binary system that can reside within the inferred dust lane of $\sim 0.5-1^{\prime \prime}$ radius (see Fig. 13). If the primary has already lost some $2 M_{\odot}$ through its stellar wind (Decin et al. 2011), the binary separation would reduce to $\sim 19$ au.

\subsection{Companion mass}

According to the method outlined by Kim \& Taam (2012a), one needs the (projected) separation between the primary star and its companion to derive the binary mass ratio. However, even after an extensive search of the literature, no direct identification of the potential binary companion was possible. We are hence lacking diagnostics to constrain the mass of the companion. However, because the ALMA PV data around PA 10-20 might be explained by a spiral shock caused by the reflex motion of the mass-losing primary star implies that the mass of the secondary cannot be very low, meaning that the secondary cannot be a Jupiter-like planet or a brown dwarf. Although the simulations of Mastrodemos \& Morris (1999) were not finetuned toward the specific situation of CW Leo (i.e., the radius of CW Leo is larger than the input radius for the model simulations, and the estimated mass of CW Leo is around $4 M_{\odot}$, while the input model mass for the primary, $M_{\mathrm{p}}$, is $1.5 M_{\odot}$ ), we can use their results as a guideline to estimate the massratio, $M_{\mathrm{s}} / M_{\mathrm{p}}$, with $M_{\mathrm{s}}$ the mass of the secondary. Their model sequence $\mathrm{M} 10 \rightarrow \mathrm{M} 17 \rightarrow \mathrm{M} 18$ shows that for a decrease of the mass of the secondary $\left(1 M_{\odot} \rightarrow 0.5 M_{\odot} \rightarrow 0.25 M_{\odot}\right)$ the morphology changes from bipolar $\rightarrow$ elliptical $\rightarrow$ quasi-spherical, meaning that the latter case resembles the signature in our ALMA data and multiple shells seen in the optical and infrared images (Mauron \& Huggins 2000; Decin et al. 2011). This would imply a mass ratio of about $1 / 6$ or a mass for the secondary around 0.6 $M_{\odot}$. Using Eq. (2) in Eggleton (1983), this implies that the effective radius of the Roche lobe, $r_{\mathrm{L}}$, is 0.54 times the orbital separation, or $r_{\mathrm{L}}$ is $\sim 13.5 \mathrm{au}\left(4.5 R_{\star}\right)$.

An alternative method was offered by Huggins et al. (2009), who used the models of Mastrodemos \& Morris (1999) to develop a prescription for the observed envelope shapes in terms of the binary parameters. The companion modifies the mass loss by gravitationally focusing the wind towards the orbital plane and thereby determines the shape of the envelope at large distances from the star. They defined the envelope shape parameter, $K_{n}$, as the density contrast between the pole and the equator

$K_{n}-1=\left(n_{\text {eq }}-n_{\text {po }}\right) / n_{\text {po }}$,

with $n_{\mathrm{eq}}$ and $n_{\mathrm{po}}$ being the density at the equator and pole. Using the results of Mastrodemos \& Morris (1999), Huggins et al. (2009) developed an empirical relation between the envelope shape parameter $K_{n}$ and the binary parameters, being the masses of the primary and secondary $\left(M_{\mathrm{p}}\right.$ and $\left.M_{\mathrm{s}}\right)$, the binary separation $(d)$ and the velocity of the wind at the orbit of the secondary $\left(V_{\mathrm{s}}\right)$. From the numerical simulations, they derived following equation (Eq. (4) in their paper):

$\log \left(K_{n}-1\right)=5.19( \pm 0.66)+1.40( \pm 0.21) \log \left(M_{\mathrm{s}} / V_{\mathrm{s}}^{2} d\right)$,

independent of the mass of the primary $M_{\mathrm{p}}$, and with $M_{\mathrm{s}}$ in units of solar mass, $V_{\mathrm{s}}$ in $\mathrm{km} \mathrm{s}^{-1}$ and $d$ in astronomical units (au). Although the models were not fine-tuned toward the specific situation of CW Leo (i.e., the radius of CW Leo is larger than the input radius for the model simulations), we can use this relation and the value of $w$, characterizing the latitudinal dependence (see Sect. 7.2.2), to derive a first crude estimate of the companion mass.
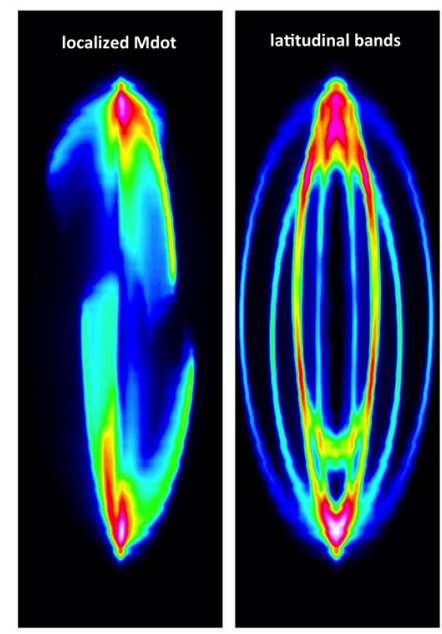

Fig. 19. Position-velocity diagrams for enhanced mass-loss ejections at $\mathrm{PA}=90^{\circ}$. Left: simulations for localized massloss ejections covering $\sim 10 \%$ of the surface at an interval of $1^{\prime \prime}$ (Dinh-V-Trung \& Lim 2008). Right: simulations for latitudinal bands according to the model of Le Bertre et al. (1989).

In Sect. 8.1 we derive a best-fit value for $w$ of $\sim 30^{\circ}$, resulting in a value of $K_{n}$ of about 10 (using Eq. (6)). Using Eq. (10), we derive that

$$
\frac{M_{\mathrm{s}}}{V_{\mathrm{s}}^{2} d}=0.8 \times 10^{-3} \frac{M_{\odot}}{\left(\mathrm{km} \mathrm{s}^{-1}\right)^{2} \mathrm{au}} .
$$

For a binary separation $d$ of $20 \mathrm{au}$, and the velocity of the wind at the orbit of the secondary, $V_{\mathrm{s}}$, being $\sim 8 \mathrm{~km} \mathrm{~s}^{-1}$ (see Fig. 5) the derived mass for the secondary, $M_{\mathrm{s}}$, is $\sim 1.1 M_{\odot}$. However, the uncertainty on the estimated value for $M_{\mathrm{s}}$ is significant because of the square dependence on the highly uncertain value of $V_{\mathrm{s}}$ and the uncertainty of $\sim 15^{\circ}$ in the value of $w$.

The derived rough estimate for the companion mass places the companion in the category of a white dwarf or an unevolved low-mass main sequence star. However, a binary with a white dwarf companion could result in a so-called dusty symbiotic system. This type of system harbours a very hot and active accretion disk, with clear signatures in the UV and many forbidden atomic lines of [O III], [Ne III], and [Fe IV] in the optical spectrum, for example. Since no signs of activity are seen in the optical/UV spectra of CW Leo, we tentatively postulate that the companion of CW Leo is an unevolved M-type dwarf.

\subsection{Other scenarios}

One might wonder if localized mass-loss ejections radially driven outward, without the action of a binary companion, might result in the type of PV diagrams seen in the ALMA data. Dinh-V-Trung \& Lim (2008) suggested that the shells seen in the VLA data of $\mathrm{HC}_{3} \mathrm{~N}$ and $\mathrm{HC}_{5} \mathrm{~N}$ at a distance of $\sim 15^{\prime \prime}$ from the central star cover some $10 \%$ of the stellar surface at the time of ejection. This scenario can be simulated using the simple parametric description for density-enhanced shells (Sect. 7.2.1), but constraining randomly the longitudinal and latitudinal coordinate for each of the five shells so that each segment covers only $\sim 10 \%$ of the surface. This suggestion provides much (parametric) freedom to model the wind of CW Leo. An example of such a PV diagram is shown in the left panel of Fig. 19. While this example shows some resemblance with the ALMA PV diagrams, this scenario would need much fine-tuning to obtain a model showing almost symmetrical signatures as seen in the ALMA PV diagram for PA $\sim 10-20^{\circ}$ (Fig. 12), but for which almost no structure is left in a PV diagram taken at a PA $90^{\circ}$ apart.

A way to constrain the huge parameter space entailed by the model for the localized mass-loss ejections is offered by the 
model presented by Le Bertre et al. (1989), who suggested that ejected material also moves as a result of the stellar rotation, creating latitudinal bands. This type of model was recently used by Jeffers et al. (2014) to explain the ExPo images of CW Leo. The SHAPE simulations for this scenario are also based on the model for the density-enhanced shells (Sect. 7.2.1), but the latitudinal coordinates of each shell only cover $\sim 5^{\circ}$. These simulations do not rule out the ejection of clumps with initial extent $\sim 10 \%$ of the stellar surface, nor the possible effects of stellar rotation, but the addition of the effects of the companion in creating a spiral provides a more robust explanation for the asymmetry in the PV diagrams when reflecting around zero-offset, for the differences seen in the PV diagrams $90^{\circ}$ apart and for the other diagnostics discussed in Sect. 6 and shown in Fig. 13.

\subsection{Further evolution}

There are numerous similarities between CW Leo and the young post-AGB star CRL 2688 (also called the Egg Nebula; Sahai et al. 1998). Both objects are carbon-rich, suggesting an initial mass higher than $3 M_{\odot}$, Although CRL 2688 is more advanced in its evolution, both objects have an optically thick circumstellar core with biconical cavities, and shell-like density enhancements are observed in scattered light as incomplete arcs. In CRL 2688 high-speed collimated jets are present with velocities of about $300 \mathrm{~km} \mathrm{~s}^{-1}$, which seem to be only powered during the last 200 years. According to Soker \& Livio (1994), different mechanisms can explain the collimated outflows in CRL 2688, each of them requiring the central star to be a binary, with the collimated outflow resulting from an accretion disk. The discovery of a spiral-arm structure in the ALMA data of CW Leo, which might be induced by a binary companion, supports the suggestion that CW Leo will evolve into an object similar to CRL 2688.

\section{Conclusions}

We have presented the first ALMA band 9 data of the inner wind region of $\mathrm{CW}$ Leo at a spatial resolution of $0.42 \times 0.24$ and with a sensitivity of $0.2-0.3 \mathrm{Jy} \mathrm{beam}^{-1}$ per $488 \mathrm{kHz}$ channel. We detected 25 emission lines, all of them centred on the dust continuum peak, which has a total (star+dust) flux density within the $3 \sigma$ contour of $5.66 \mathrm{Jy}$. The images prove that the vibrational lines are excited just above the stellar photosphere and that $\mathrm{SiO}$ is a parent molecule, formed close to the stellar surface, probably by shock-induced non-equilibrium chemistry. The velocity traced by the line width of the emission lines implies a steep increase of the wind velocity starting at around $5 R_{\star}$, almost reaching the terminal velocity at $\sim 11 R_{\star}$.

Both the dust emission and the emission of the brightest lines show a clear asymmetric distribution. We note, however, that the incomplete UV-coverage partly limits the quality of the data. The PV maps at different position angles of the ${ }^{13} \mathrm{CO} J=6-5$ line display correlated arc-like structures, which can be explained by a spiral arm. This spiral arm can be caused by a binary companion. Using the SHAPE modeling tool, we modelled the ${ }^{13} \mathrm{CO}$ $J=6-5 \mathrm{PV}$ diagrams. We deduce that the orbital axis lies at a position angle of $\sim 10-20^{\circ}$ north-east and that the spiral arm spacing is $\sim 1.12^{\prime \prime}$. At a distance of $150 \mathrm{pc}$, this leads to an orbital period of $55 \mathrm{yr}$ and a binary separation of $\sim 20-25$ au (or $\sim 6-8 R_{\star}$ ). We tentatively suggest that the companion is an unevolved low-mass main-sequence star.

The scenario of a binary system can explain (1) the spiral arm structure seen in the ALMA PV data; (2) the signature of a bipolar structure seen at arcsecond scales; and (3) the multiple non-concentric shells detected in the outer wind, which might represent the limb-brightened edges of the spiral arms seen almost edge-on. Granted ALMA cycle 1, time will increase the $\mathrm{S} / \mathrm{N}$ and UV-coverage, hence facilitating the in-depth interpretation of the ALMA data.

Acknowledgements. This paper makes use of the following ALMA data: ADS/JAO.ALMA\#2011.0.00277.S. ALMA is a partnership of ESO (representing its member states), NSF (USA) and NINS (Japan), together with NRC (Canada) and NSC and ASIAA (Taiwan), in cooperation with the Republic of Chile. The Joint ALMA Observatory is operated by ESO, AUI/NRAO and NAOJ. W.S. acknowledges support by grant UNAM-PAPIIT 101014. The authors thank Nicholas Koning and Miguel Santander-García for technical support with SHAPE.

\section{References}

Agúndez, M., Cernicharo, J., \& Guélin, M. 2010, ApJ, 724, L133

Castor, J. I. 1970, MNRAS, 149, 111

Cernicharo, J., Waters, L. B. F. M., Decin, L., et al. 2010, A\&A, 521, L8

Cherchneff, I. 2011, A\&A, 526, L11

Cordiner, M. A., \& Millar, T. J. 2009, ApJ, 697, 68

Crosas, M., \& Menten, K. M. 1997, ApJ, 483, 913

De Beck, E., Decin, L., de Koter, A., et al. 2010, A\&A, 523, A18

De Beck, E., Lombaert, R., Agúndez, M., et al. 2012, A\&A, 539, A108

de Graauw, T., Helmich, F. P., Phillips, T. G., et al. 2010, A\&A, 518, L6

Decin, L., Hony, S., de Koter, A., et al. 2006, A\&A, 456, 549

Decin, L., Agúndez, M., Barlow, M. J., et al. 2010a, Nature, 467, 64

Decin, L., Cernicharo, J., Barlow, M. J., et al. 2010b, A\&A, 518, L143

Decin, L., Royer, P., Cox, N. L. J., et al. 2011, A\&A, 534, A1

Dinh-V-Trung, \& Lim, J. 2008, ApJ, 678, 303

Dinh-V.-Trung, \& Lim, J. 2009, ApJ, 701, 292

Dyck, H. M., Howell, R. R., Zuckerman, B., \& Beckwith, S. 1987, PASP, 99, 99

Eggleton, P. P. 1983, ApJ, 268, 368

Fonfría, J. P., Cernicharo, J., Richter, M. J., \& Lacy, J. H. 2008, ApJ, 673, 445

Fong, D., Meixner, M., \& Shah, R. Y. 2003, ApJ, 582, L39

Freytag, B., \& Höfner, S. 2008, A\&A, 483, 571

Girart, J. M., Patel, N., Vlemmings, W. H. T., \& Rao, R. 2012, ApJ, 751, L20

Groenewegen, M. A. T. 1997, A\&A, 317, 503

Groenewegen, M. A. T., van der Veen, W. E. C. J., Lefloch, B., \& Omont, A. 1997, A\&A, 322, L21

Groenewegen, M. A. T., Barlow, M. J., Blommaert, J. A. D. L., et al. 2012, A\&A, 543, L8

Guélin, M., Lucas, R., \& Cernicharo, J. 1993, A\&A, 280, L19

Guélin, M., Forestini, M., Valiron, P., et al. 1995, A\&A, 297, 183

Höfner, S. 2008, A\&A, 491, L1

Huggins, P. J., Mauron, N., \& Wirth, E. A. 2009, MNRAS, 396, 1805

Jeffers, S. V., Min, M., Waters, L. B. F. M., et al. 2014, A\&A, 572, A3

Jenness, T., Stevens, J. A., Archibald, E. N., et al. 2002, MNRAS, 336, 14

Kastner, J. H., \& Weintraub, D. A. 1994, ApJ, 434, 719

Keady, J. J., Hall, D. N. B., \& Ridgway, S. T. 1988, ApJ, 326, 832

Kim, H., \& Taam, R. E. 2012a, ApJ, 759, L22

Kim, H., \& Taam, R. E. 2012b, ApJ, 744, 136

Kim, H., \& Taam, R. E. 2012c, ApJ, 759, 59

Kim, H., Hsieh, I.-T., Liu, S.-Y., \& Taam, R. E. 2013, ApJ, 776, 86

Ladjal, D., Barlow, M. J., Groenewegen, M. A. T., et al. 2010, A\&A, 518, L141

Lagadec, E., Zijlstra, A. A., Matsuura, M., et al. 2008, MNRAS, 383, 399

Leão, I. C., de Laverny, P., Mékarnia, D., de Medeiros, J. R., \& Vandame, B. 2006, A\&A, 455, 187

Le Bertre, T., Magain, P., \& Remy, M. 1989, The Messenger, 55, 25

Lucas, R., \& Guélin, M. 1999, in Asymptotic Giant Branch Stars, eds. T. Le Bertre, A. Lebre, \& C. Waelkens, IAU Symp., 191, 305

Maercker, M., Mohamed, S., Vlemmings, W. H. T., et al. 2012, Nature, 490, 232

Mastrodemos, N., \& Morris, M. 1999, ApJ, 523, 357

Mauron, N., \& Huggins, P. J. 1999, A\&A, 349, 203

Mauron, N., \& Huggins, P. J. 2000, A\&A, 359, 707

Mauron, N., \& Huggins, P. J. 2006, A\&A, 452, 257

McMullin, J. P., Waters, B., Schiebel, D., Young, W., \& Golap, K. 2007, in Astronomical Data Analysis Software and Systems XVI, eds. R. A. Shaw, F. Hill, \& D. J. Bell, ASP Conf. Ser., 376, 127

Mennella, V., Brucato, J. R., Colangeli, L., et al. 1998, ApJ, 496, 1058

Men'shchikov, A. B., Balega, Y., Blöcker, T., Osterbart, R., \& Weigelt, G. 2001, A\&A, 368, 497 
L. Decin et al.: Edge-on spiral detected with ALMA in the inner wind of CW Leo

Menten, K. M., Reid, M. J., Krügel, E., Claussen, M. J., \& Sahai, R. 2006, A\&A, 453, 301

Menten, K. M., Reid, M. J., Kamiński, T., \& Claussen, M. J. 2012, A\&A, 543, A73

Menut, J., Gendron, E., Schartmann, M., et al. 2007, MNRAS, 376, L6

Morris, M. 1987, PASP, 99, 1115

Murakawa, K., Suto, H., Oya, S., et al. 2005, A\&A, 436, 601

Olofsson, H., Eriksson, K., Gustafsson, B., \& Carlstrom, U. 1993, ApJS, 87, 267

Patel, N. A., Young, K. H., Gottlieb, C. A., et al. 2011, ApJS, 193, 17

Perley, R. A. 1999, in Synthesis Imaging in Radio Astronomy II, eds.

G. B. Taylor, C. L. Carilli, \& R. A. Perley, ASP Conf. Ser., 180, 275

Reid, M. J., \& Menten, K. M. 1997, ApJ, 476, 327

Roelfsema, P. R., Helmich, F. P., Teyssier, D., et al. 2012, A\&A, 537, A17

Sahai, R., Trauger, J. T., Watson, A. M., et al. 1998, ApJ, 493, 301

Santander-García, M., Bujarrabal, V., \& Alcolea, J. 2012, A\&A, 545, A114

Santander-García, M., Bujarrabal, V., Koning, N., \& Steffen, W. 2015, A\&A, 573, A56
Schöier, F. L., van der Tak, F. F. S., van Dishoeck, E. F., \& Black, J. H. 2005 , A\&A, 432, 369

Skinner, C. J., Meixner, M., \& Bobrowsky, M. 1998, MNRAS, 300, L29

Soker, N., \& Clayton, G. C. 1999, MNRAS, 307, 993

Soker, N., \& Livio, M. 1994, ApJ, 421, 219

Steffen, W., \& López, J. A. 2006, Rev. Mexicana Astron. Astrofis., 42, 99

Steffen, W., Koning, N., Wenger, S., Morisset, C., \& Magnor, M. 2011, IEEE Transactions on Visualization and Computer Graphics, 17, 454

Teyssier, D., Cernicharo, J., Quintana-Lacaci, G., et al. 2013, The Universe explored by Herschel

Tuthill, P. G., Monnier, J. D., Danchi, W. C., \& Lopez, B. 2000, ApJ, 543, 284

Weigelt, G., Balega, Y. Y., Blöcker, T., et al. 2002, A\&A, 392, 131

Williams, P. G., \& White, G. J. 1992, A\&A, 266, 365

Wittkowski, M., Boboltz, D. A., Ireland, M., et al. 2011, A\&A, 532, L7

Woitke, P. 2006, A\&A, 452, 537

Young, K. H., Hunter, T. R., Wilner, D. J., et al. 2004, ApJ, 616, L51 


\section{Appendix A: Position-velocity diagrams}
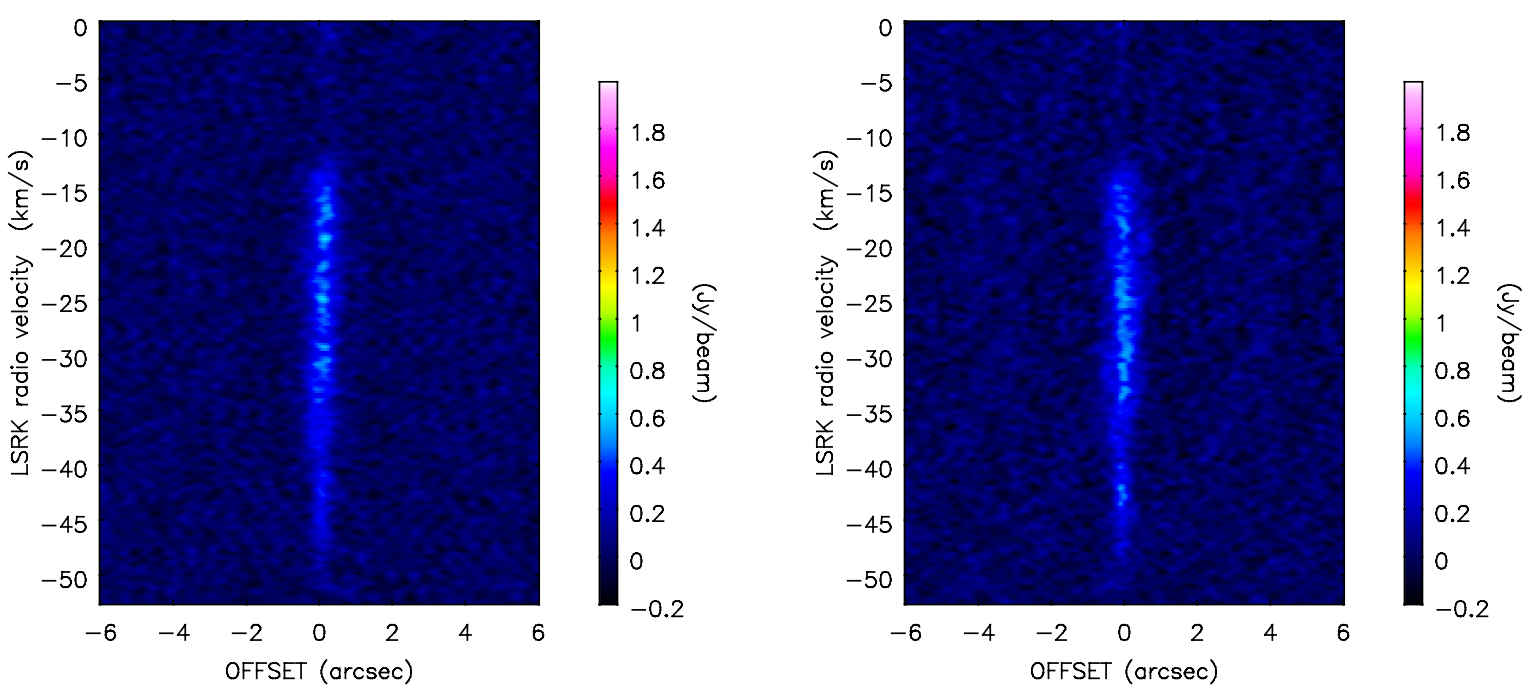

Fig. A.1. Position-velocity diagram along the right ascension $\left(\mathrm{PA}=90^{\circ}\right.$, left $)$ and declination $\left(\mathrm{PA}=0^{\circ}\right.$, right $)$ of ${ }^{29} \mathrm{SiO} J=15-14$ at $642.808 \mathrm{GHz}$; blended with $\mathrm{NaCN} 24(7,17)-24(6,18)$ at $642.826 \mathrm{GHz}$ and $\mathrm{NaCN} 24(7,18)-24(6,19)$ at $642.832 \mathrm{GHz}$.
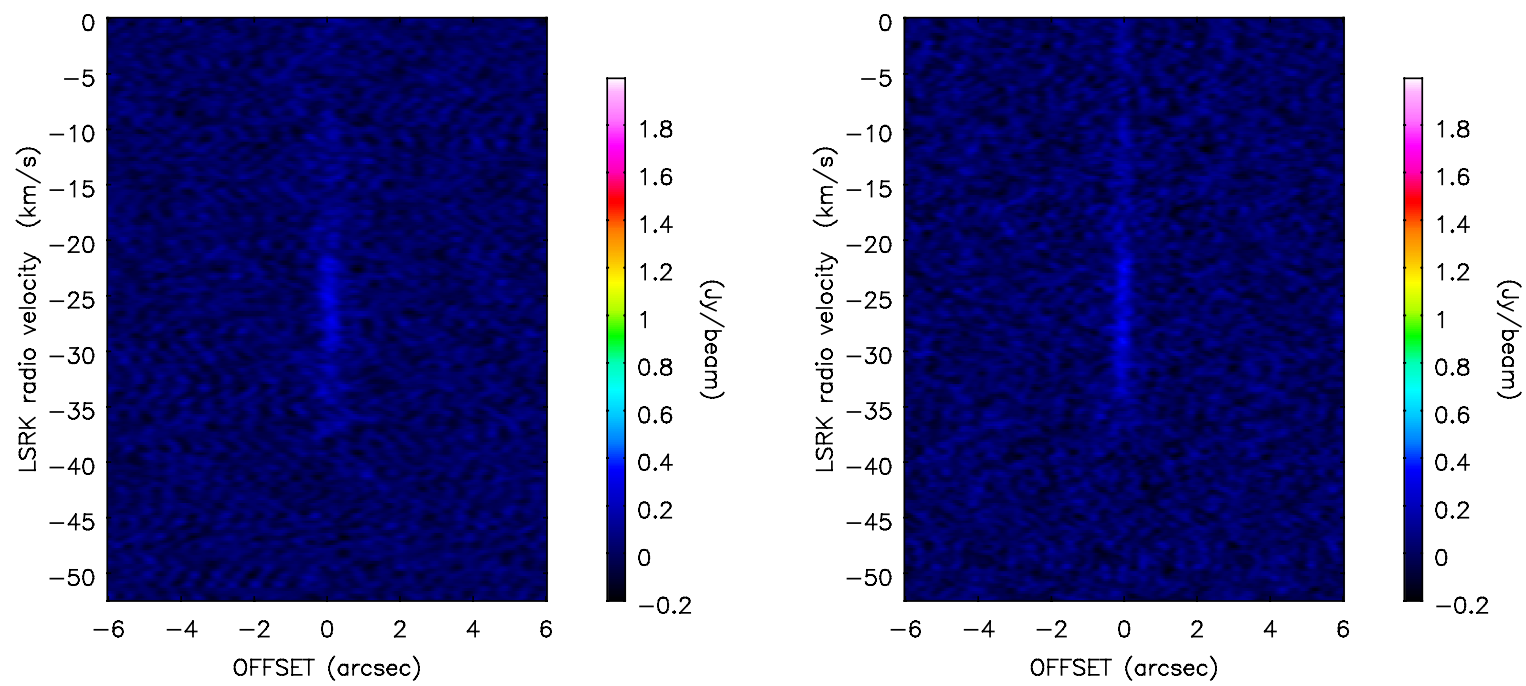

Fig. A.2. Position-velocity diagram along the right ascension $\left(\mathrm{PA}=90^{\circ}\right.$, left $)$ and declination $\left(\mathrm{PA}=0^{\circ}\right.$, right $)$ of $\mathrm{Si}^{33} \mathrm{~S} J=36-35$ at $643.260 \mathrm{GHz}$.
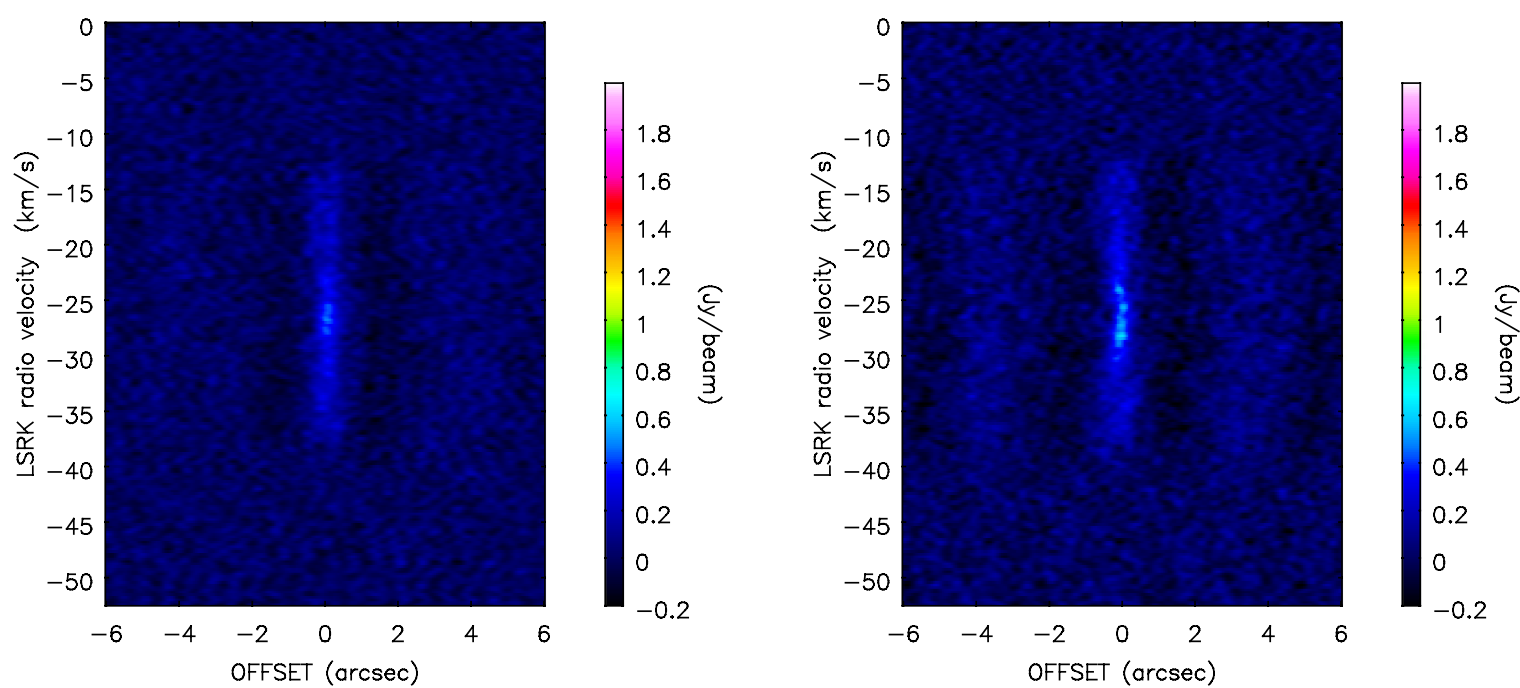

Fig. A.3. Position-velocity diagram along the right ascension $\left(\mathrm{PA}=90^{\circ}\right.$, left $)$ and declination $\left(\mathrm{PA}=0^{\circ}\right.$, right $)$ of $\mathrm{SiC}_{2} 26(4,22)-25(4,21)$ at $645.752 \mathrm{GHz}$. 
L. Decin et al.: Edge-on spiral detected with ALMA in the inner wind of CW Leo
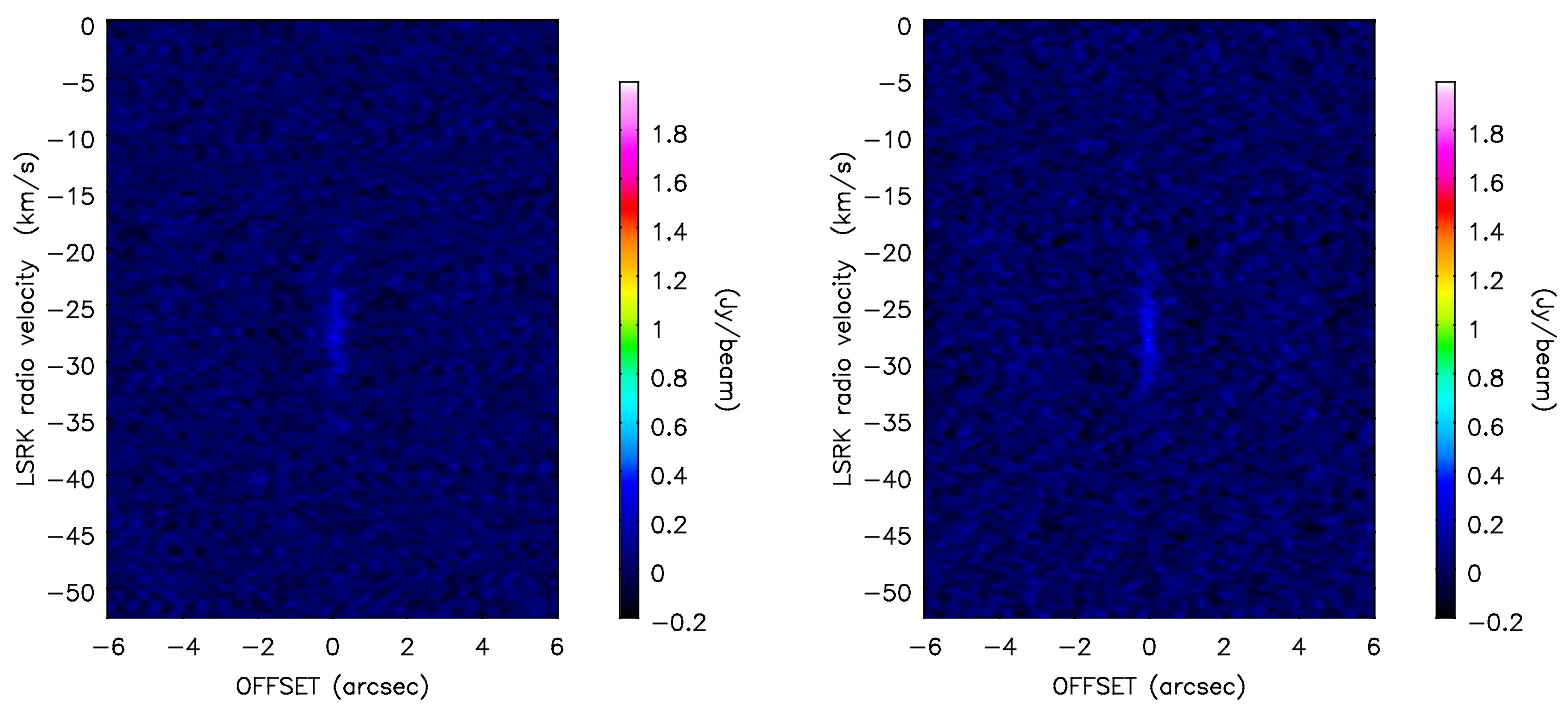

Fig. A.4. Position-velocity diagram along the right ascension $\left(\mathrm{PA}=90^{\circ}\right.$, left $)$ and declination $\left(\mathrm{PA}=0^{\circ}\right.$, right $)$ of $\mathrm{Si}^{34} \mathrm{~S} v=2 J=37-36$ at $645.919 \mathrm{GHz}$.
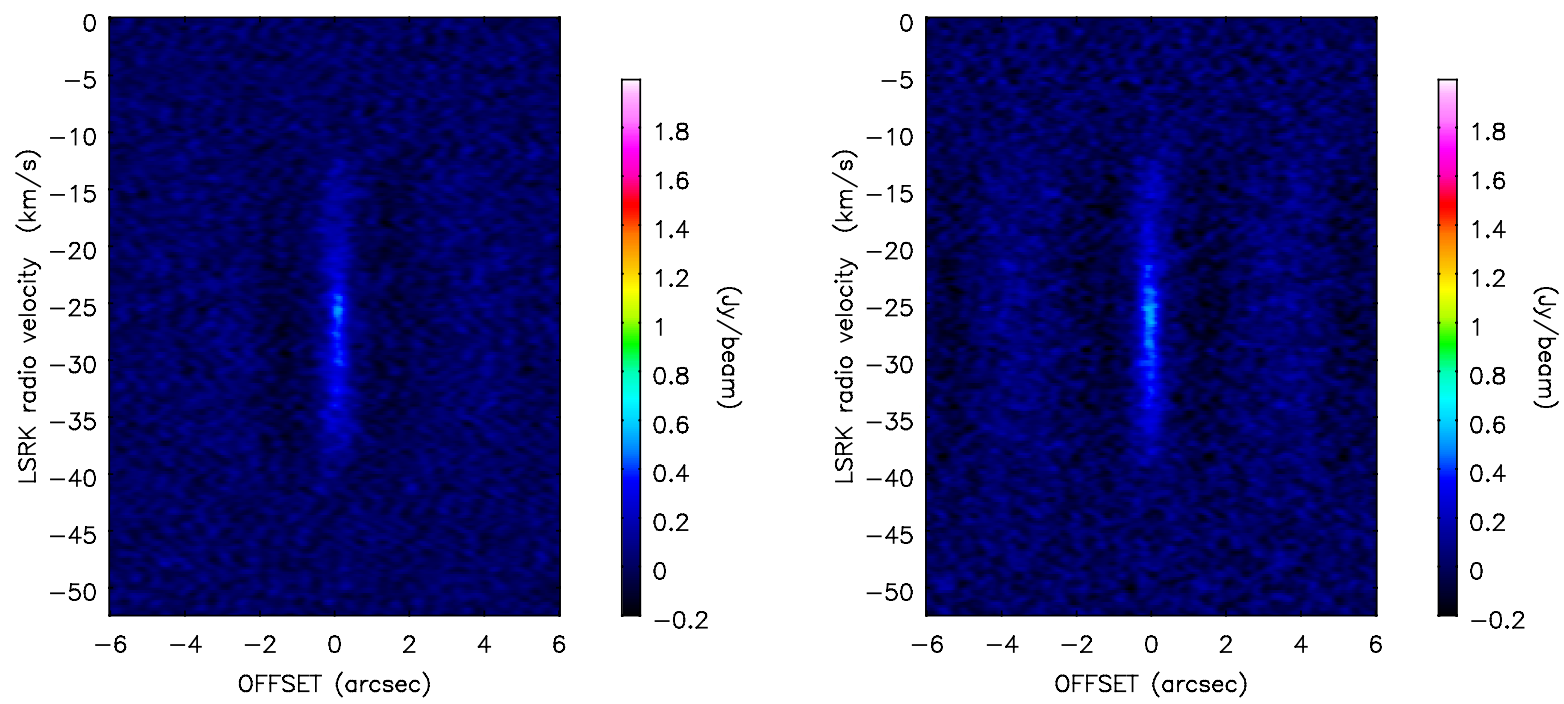

Fig. A.5. Position-velocity diagram along the right ascension $\left(\mathrm{PA}=90^{\circ}\right.$, left $)$ and declination $\left(\mathrm{PA}=0^{\circ}\right.$, right $)$ of $\mathrm{SiC}_{2} 27(6,22)-26(6,21)$ at $646.323 \mathrm{GHz}$, strongly blended by the $\mathrm{SiO} v=1 J=15-14$ line at $646.429 \mathrm{GHz}$.
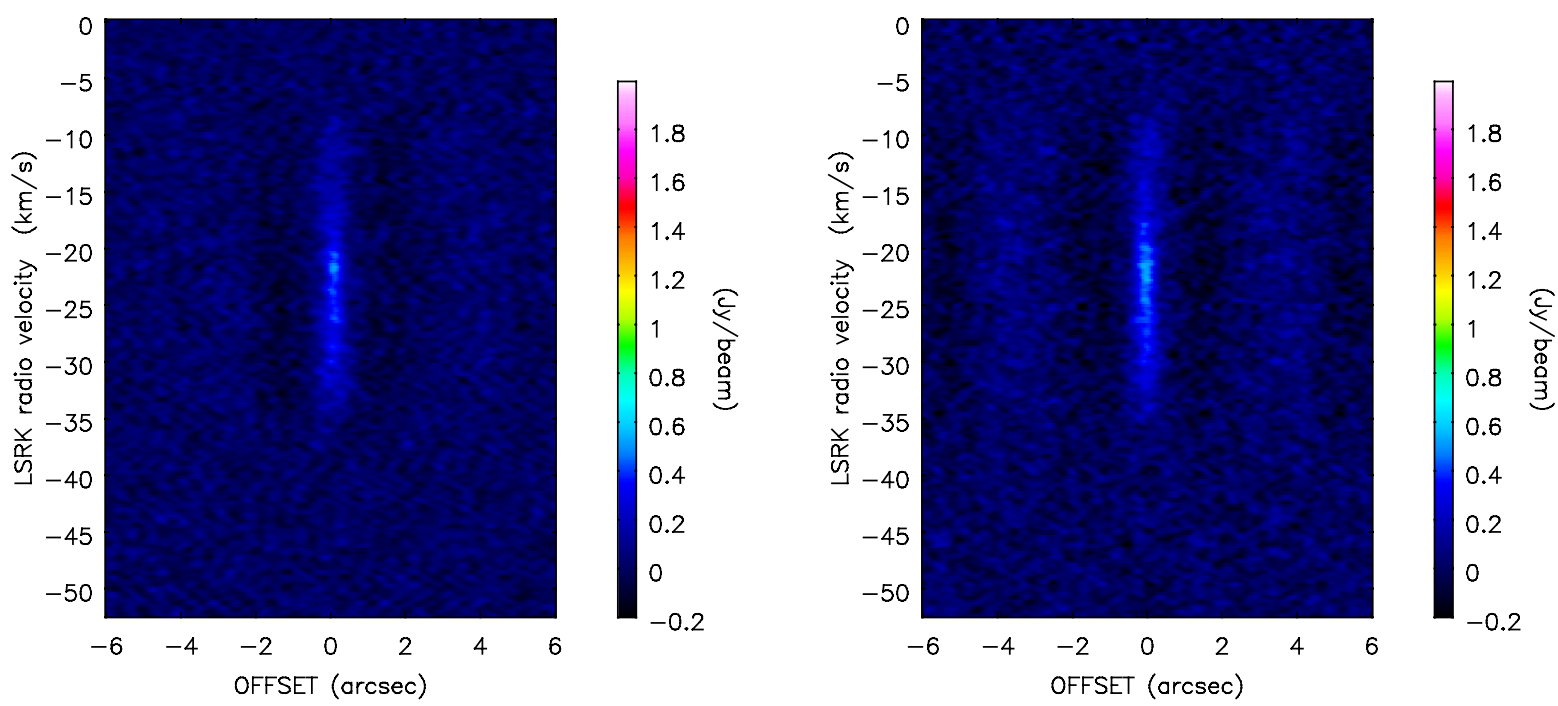

Fig. A.6. Position-velocity diagram along the right ascension $\left(\mathrm{PA}=90^{\circ}\right.$, left $)$ and declination $\left(\mathrm{PA}=0^{\circ}\right.$, right $)$ of $\mathrm{SiO} v=1 J=15-14$ at 646.429 GHz, blended with $\mathrm{SiC}_{2} 27(6,22)-26(6,21)$ at $646.323 \mathrm{GHz}$. 
A\&A 574, A5 (2015)
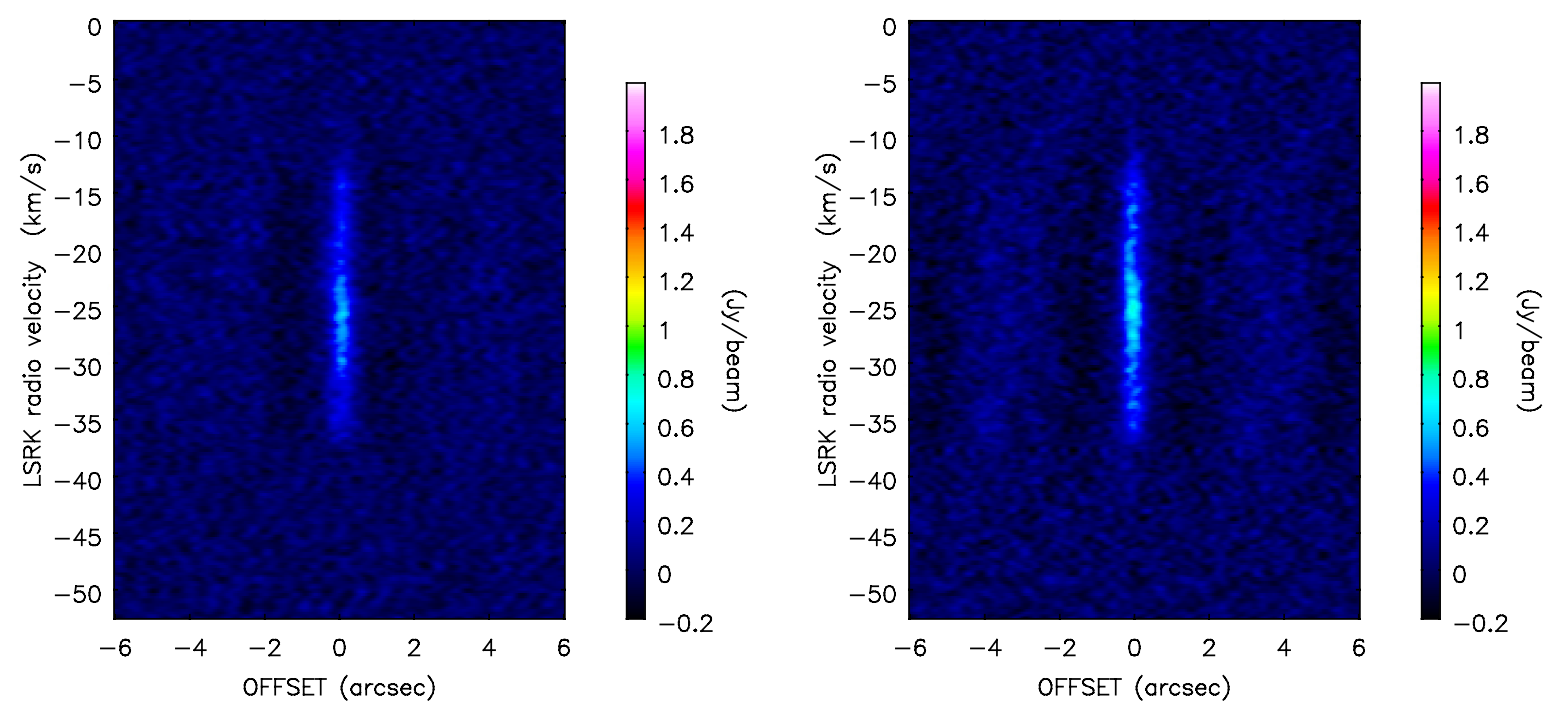

Fig. A.7. Position-velocity diagram along the right ascension $\left(\mathrm{PA}=90^{\circ}\right.$, left $)$ and declination $\left(\mathrm{PA}=0^{\circ}\right.$, right $)$ of ${ }^{30} \mathrm{SiS} J=37-36$ at $646.770 \mathrm{GHz}$.
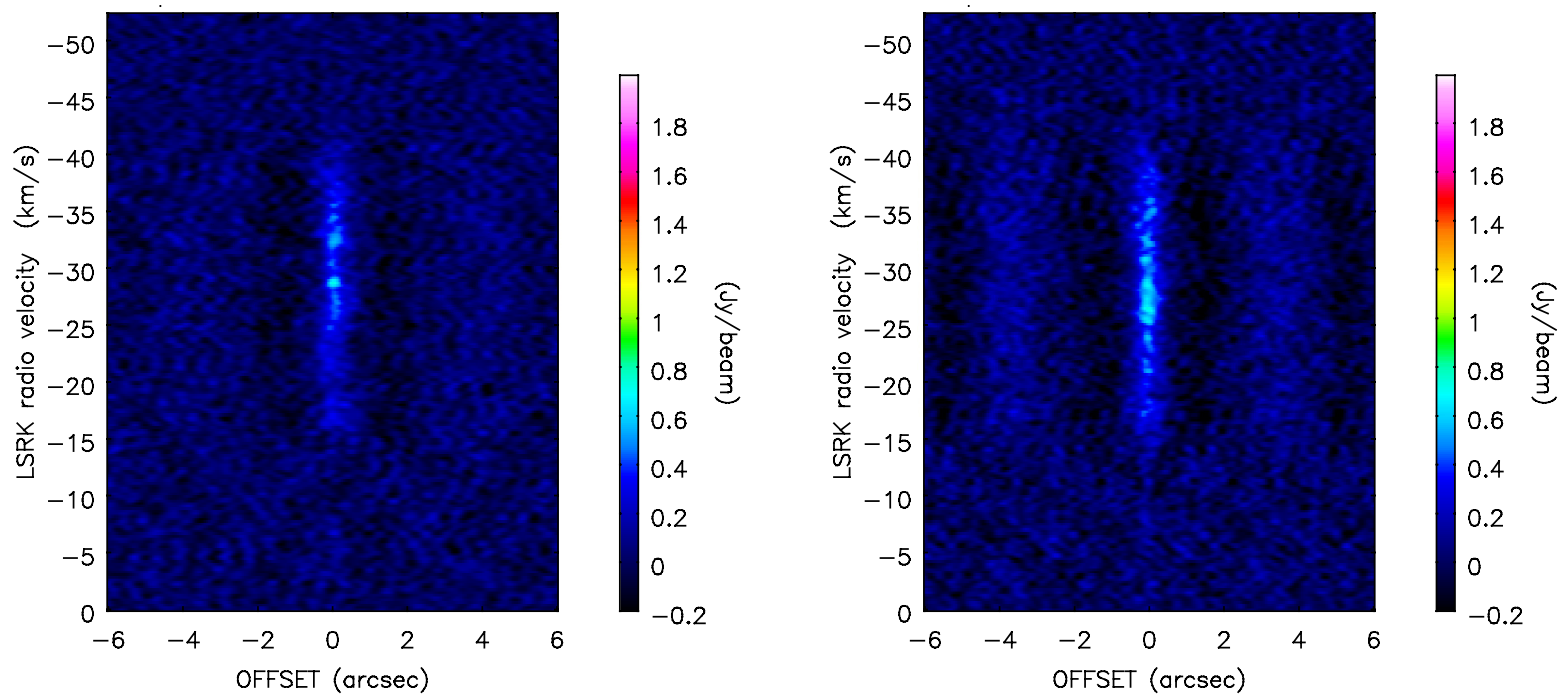

Fig. A.8. Position-velocity diagram along the right ascension $\left(\mathrm{PA}=90^{\circ}\right.$, left $)$ and declination $\left(\mathrm{PA}=0^{\circ}\right.$, right $)$ of ${ }^{29} \mathrm{Si}^{34} \mathrm{~S} J=38-37$ at $657.124 \mathrm{GHz}$, blended with $\mathrm{SiC}_{2} 31(0,31)-30(0,30)$ at $657.128 \mathrm{GHz}$.
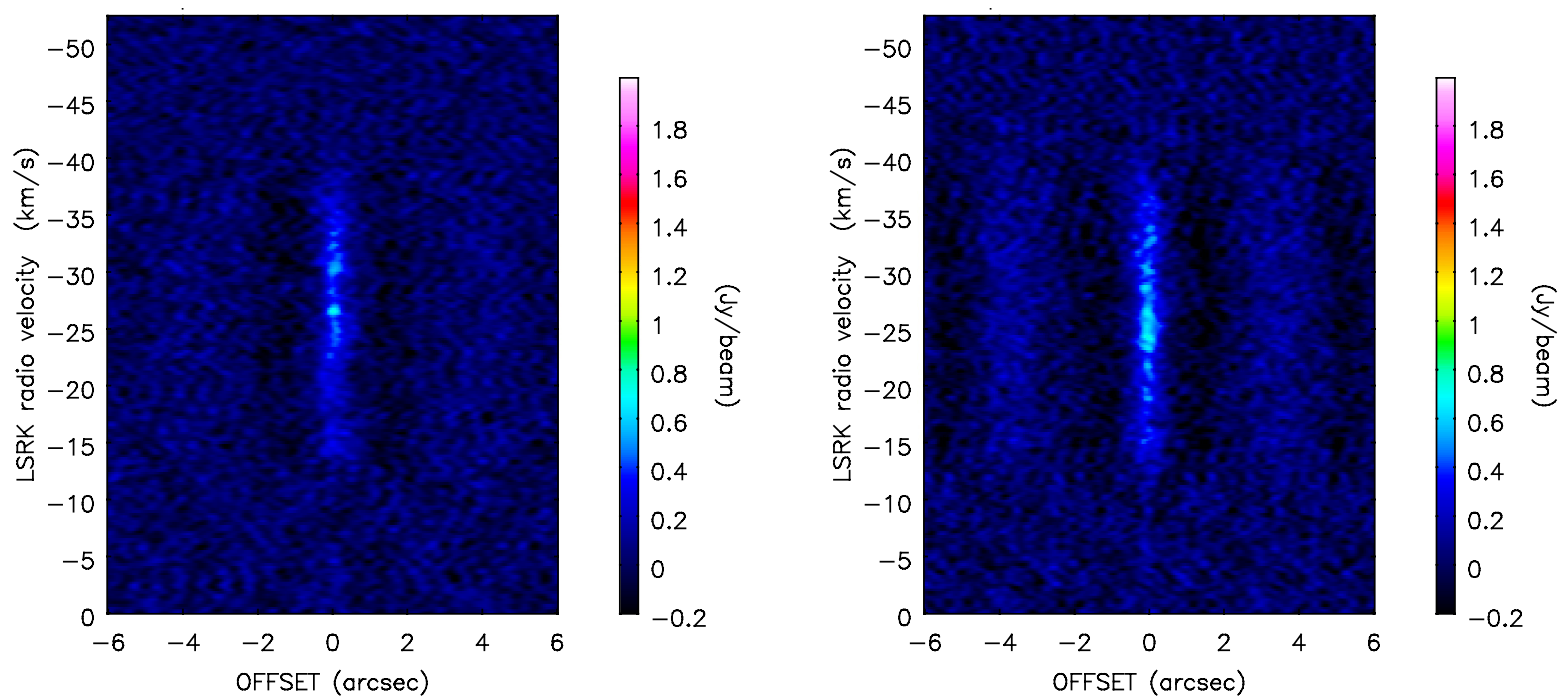

Fig. A.9. Position-velocity diagram along the right ascension $\left(\mathrm{PA}=90^{\circ}\right.$, left $)$ and declination $\left(\mathrm{PA}=0^{\circ}\right.$, right $)$ of $\mathrm{SiC}_{2} 31(0,31)-30(0,30)$ at $657.128 \mathrm{GHz}$, blended with ${ }^{29} \mathrm{Si}^{34} \mathrm{~S} J=38-37$ at $657.124 \mathrm{GHz}$. 
L. Decin et al.: Edge-on spiral detected with ALMA in the inner wind of CW Leo
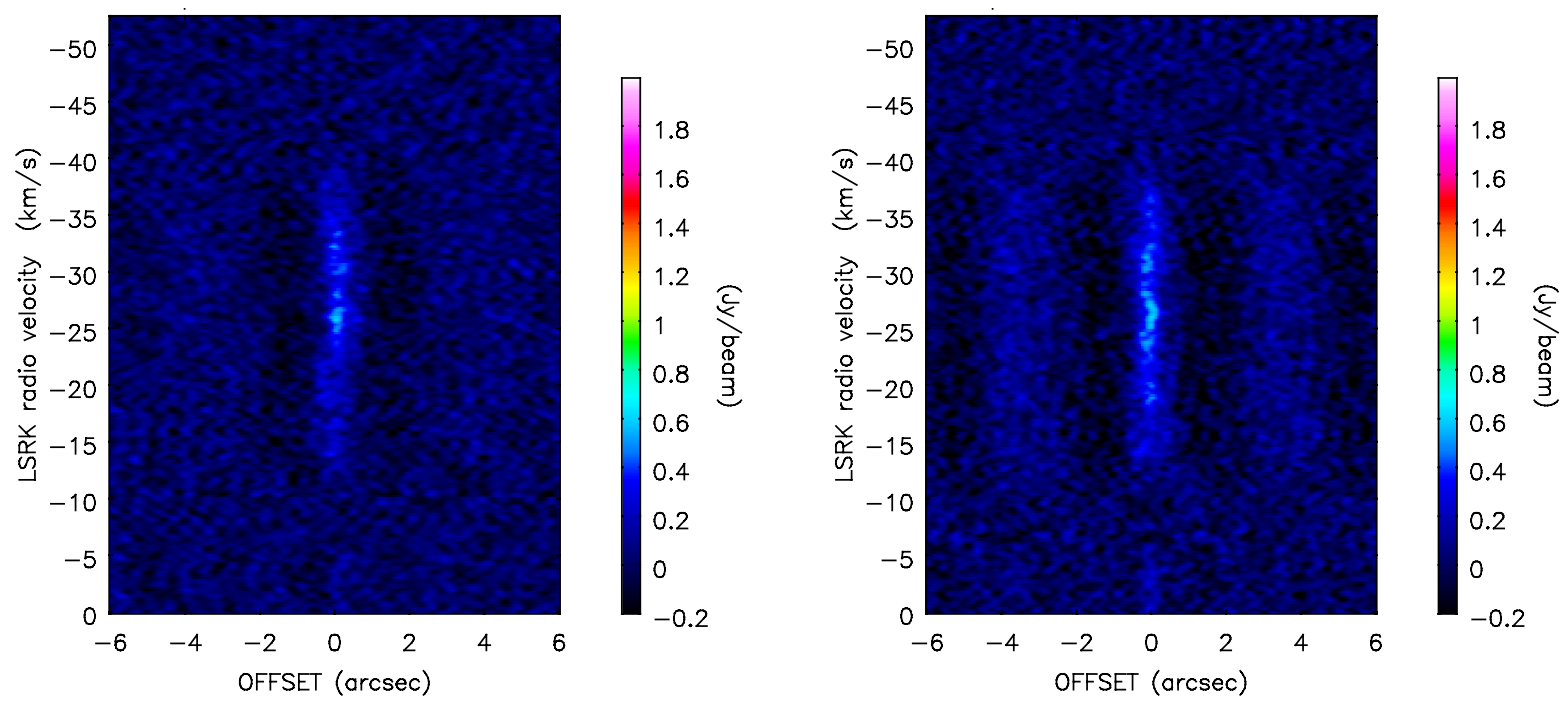

Fig. A.10. Position-velocity diagram along the right ascension $\left(\mathrm{PA}=90^{\circ}\right.$, left $)$ and declination $\left(\mathrm{PA}=0^{\circ}\right.$, right $)$ of $\mathrm{SiC} \mathrm{C}_{2} 30(2,29)-29(2,28)$ at $657.603 \mathrm{GHz}$.
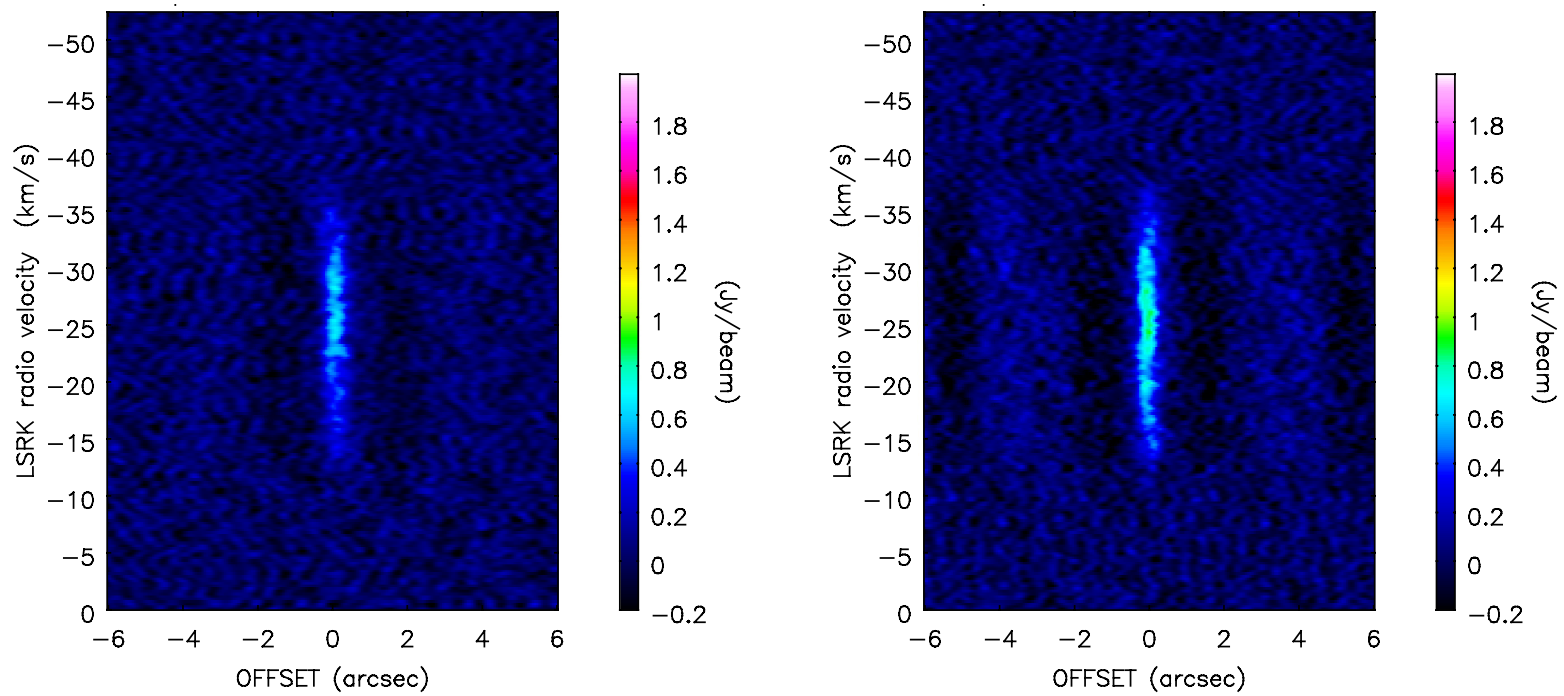

Fig. A.11. Position-velocity diagram along the right ascension $\left(\mathrm{PA}=90^{\circ}\right.$, left $)$ and declination $\left(\mathrm{PA}=0^{\circ}\right.$, right $)$ of ${ }^{29} \mathrm{SiS} J=37-36$ at $658.224 \mathrm{GHz}$.
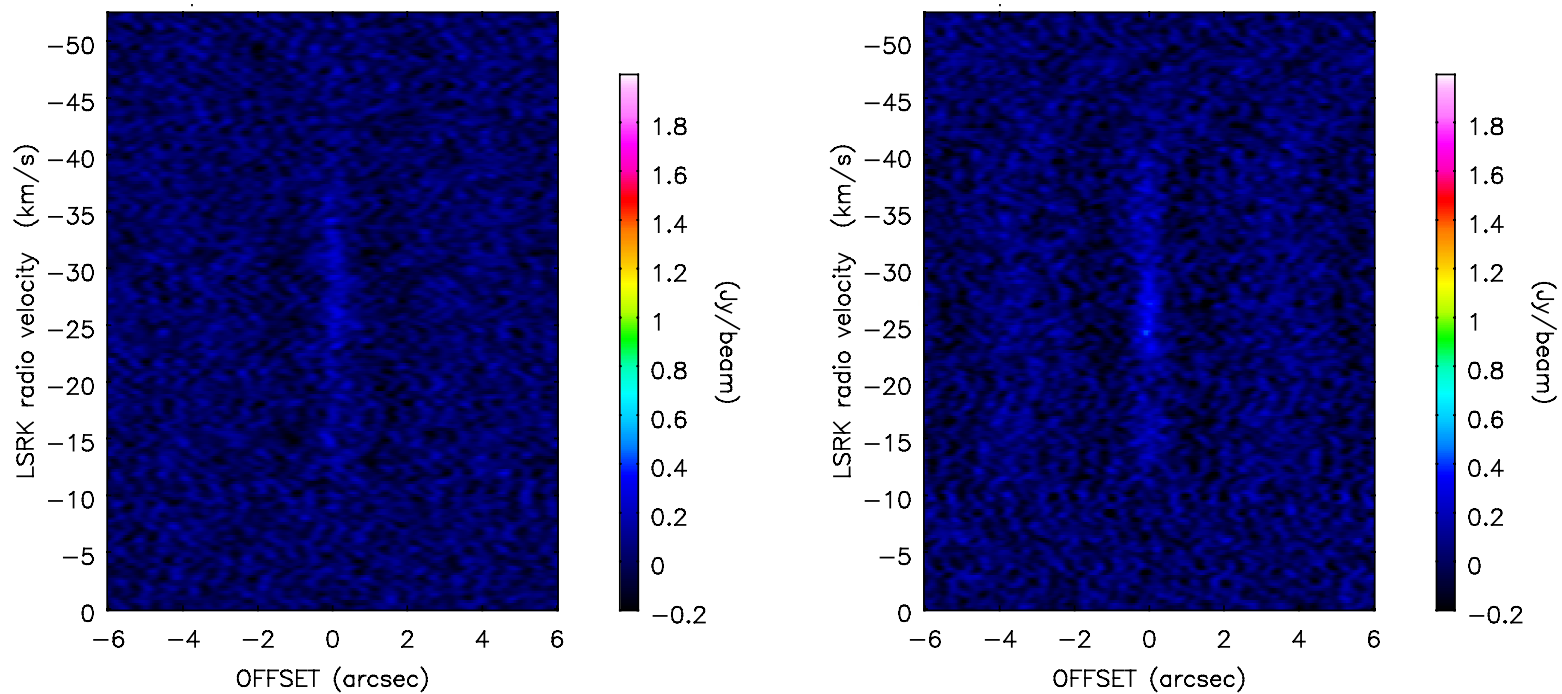

Fig. A.12. Position-velocity diagram along the right ascension $\left(\mathrm{PA}=90^{\circ}\right.$, left $)$ and declination $\left(\mathrm{PA}=0^{\circ}\right.$, right $)$ of $\mathrm{AlF} J=20-19$ at $658.529 \mathrm{GHz}$, weakly blended with $\mathrm{C}^{18} \mathrm{O} J=6-5$ at $658.553 \mathrm{GHz}$. 

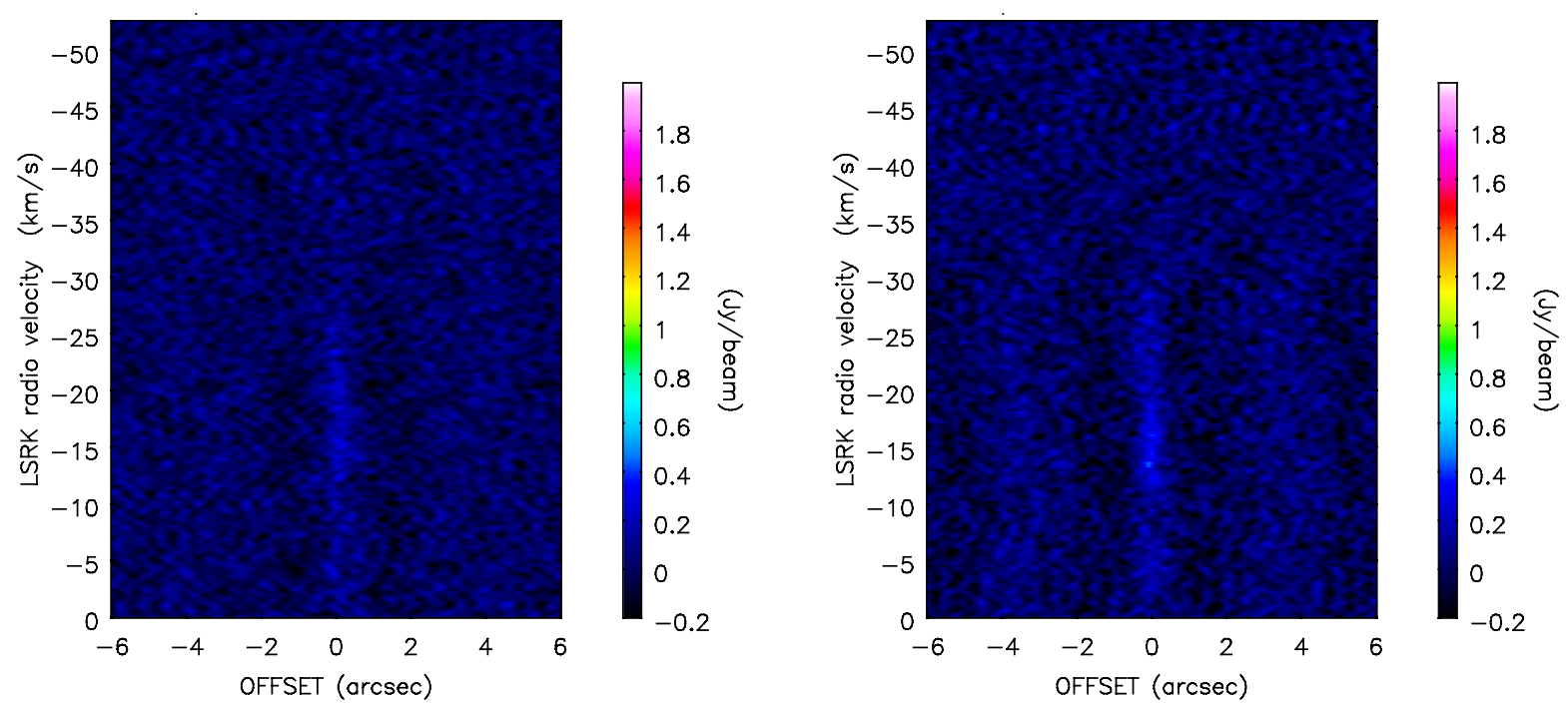

Fig. A.13. Position-velocity diagram along the right ascension $\left(\mathrm{PA}=90^{\circ}\right.$, left $)$ and declination $\left(\mathrm{PA}=0^{\circ}\right.$, right $)$ of $\mathrm{C}^{18} \mathrm{O} J=6-5$ at $658.553 \mathrm{GHz}$. Main emission comes from AlF $J=20-19$ at $658.529 \mathrm{GHz}$ (i.e. shifted by $10 \mathrm{~km} \mathrm{~s}^{-1}$ ).
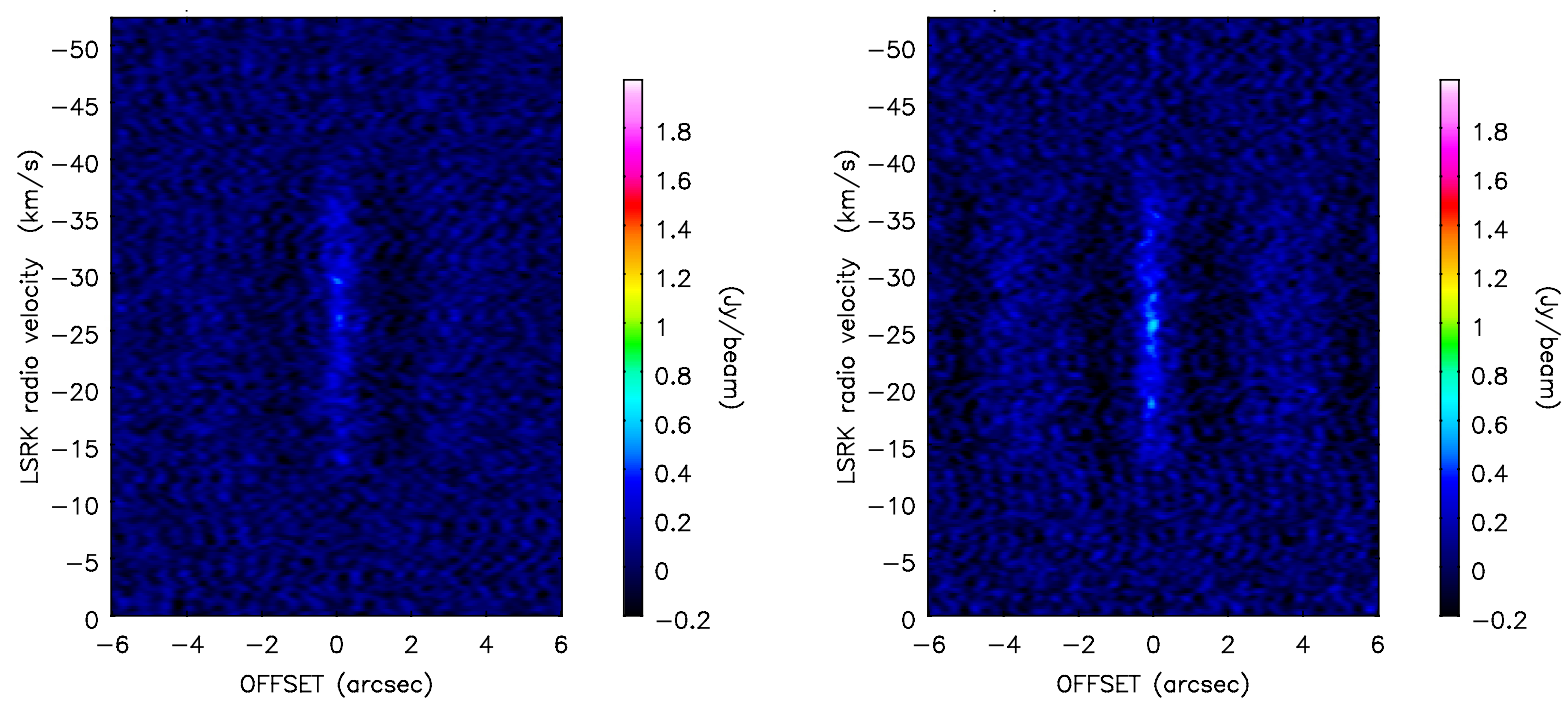

Fig. A.14. Position-velocity diagram along the right ascension $\left(\mathrm{PA}=90^{\circ}\right.$, left $)$ and declination $\left(\mathrm{PA}=0^{\circ}\right.$, right $)$ of $\mathrm{SiC}_{2} 29(2,27)-28(2,26)$ at $658.792 \mathrm{GHz}$. 